\title{
Asymptotic expansion of low-energy excitations for weakly interacting bosons
}

\author{
Lea Boßmann ${ }^{\mathbb{D}} 1$, Sören Petrat ${ }^{(\mathbb{D} 2,3}$ and Robert Seiringer ${ }^{(\mathbb{D}} 4$ \\ ${ }^{1}$ Institute of Science and Technology Austria, Am Campus 1, 3400 Klosterneuburg, Austria; E-mail: lea.bossmann@ist.ac.at. \\ ${ }^{2}$ Department of Mathematics and Logistics, Jacobs University Bremen, Campus Ring 1, 28759 Bremen, Germany; \\ E-mail: s.petrat@jacobs-university.de. \\ ${ }^{3}$ University of Bremen, Department 3 - Mathematics, Bibliothekstr. 5, 28359 Bremen, Germany. \\ ${ }^{4}$ Institute of Science and Technology Austria, Am Campus 1, 3400 Klosterneuburg, Austria; E-mail: robert.seiringer@ist.ac.at.
}

Received: 27 October 2020; Revised: 10 February 2021; Accepted: 26 February 2021

2020 Mathematics Subject Classification: Primary - 81V73; Secondary - 81Q10

\begin{abstract}
We consider a system of $N$ bosons in the mean-field scaling regime for a class of interactions including the repulsive Coulomb potential. We derive an asymptotic expansion of the low-energy eigenstates and the corresponding energies, which provides corrections to Bogoliubov theory to any order in $1 / N$.
\end{abstract}

\section{Introduction}

We consider a system of $N$ interacting bosons in $\mathbb{R}^{d}, d \geq 1$, which are described by the $N$-body Hamiltonian

$$
H_{N}=\sum_{j=1}^{N}\left(-\Delta_{j}+V^{\mathrm{ext}}\left(x_{j}\right)\right)+\lambda_{N} \sum_{1 \leq i<j \leq N} v\left(x_{i}-x_{j}\right)
$$

with coupling parameter

$$
\lambda_{N}:=\frac{1}{N-1}
$$

corresponding to a mean-field (or Hartree) regime of weak and long-range interactions. The Hamiltonian $H_{N}$ acts on the Hilbert space of square-integrable, permutation-symmetric functions on $\mathbb{R}^{d N}$,

$$
\mathfrak{H}_{\mathrm{sym}}^{N}:=\bigotimes_{\mathrm{sym}}^{N} \mathfrak{H}, \quad \mathfrak{H}:=L^{2}\left(\mathbb{R}^{d}\right)
$$

Our assumptions on the interaction $v$ include the repulsive Coulomb potential $(d=3)$, and our conditions on the external trap $V^{\text {ext }}$ are satisfied, for example, by harmonic potentials. We study the spectrum ${ }^{1}$ of $H_{N}$

$$
\mathscr{E}_{N}^{(0)}<\mathscr{E}_{N}^{(1)}<\cdots<\mathscr{E}_{N}^{(n)}<\ldots
$$

${ }^{1}$ We follow the convention of counting eigenvalues without multiplicity.

(C) The Author(s), 2021. Published by Cambridge University Press. This is an Open Access article, distributed under the terms of the Creative Commons Attribution licence (http://creativecommons.org/licenses/by/4.0/), which permits unrestricted re-use, distribution, and reproduction in any medium, provided the original work is properly cited. 
for excitation energies of order 1 above the ground state, as well as the corresponding eigenfunctions. Our main result is an asymptotic expansion of the eigenvalues of $H_{N}$, which, in the case where the degeneracy does not change in the limit $N \rightarrow \infty$, reads

$$
\mathscr{E}_{N}^{(n)}=N e_{\mathrm{H}}+E_{0}^{(n)}+\lambda_{N} E_{1}^{(n)}+\lambda_{N}^{2} E_{2}^{(n)}+\lambda_{N}^{3} E_{3}^{(n)}+\cdots,
$$

where the $N$-dependence is exclusively in the prefactors $N$ and $\lambda_{N}$. More precisely, we construct an asymptotic expansion of the spectral projectors of $H_{N}$, which implies equation (1.2). For eigenvalues whose degeneracy increases in the limit $N \rightarrow \infty$, we obtain a comparable result for the sum of those eigenvalues that become degenerate in the limit.

Let us explain the different contributions in equation (1.2). It is well known (see, e.g., [65, 27, 34, 36, 39]) that for any fixed $n \in \mathbb{N}_{0}$, the eigenstates $\Psi_{N}^{(n)}$ of $H_{N}$ associated with $\mathscr{E}_{N}^{(n)}$ exhibit Bose-Einstein condensation in the minimiser $\varphi$ of the Hartree functional. As equation (1.1) describes a mean-field regime, the leading order in equation (1.2) is given by

$$
\mathscr{E}_{N}^{(n)}=\left\langle\Psi_{N}^{(n)}, H_{N} \Psi_{N}^{(n)}\right\rangle=\left\langle\varphi^{\otimes N}, H_{N} \varphi^{\otimes N}\right\rangle+\mathcal{O}(1)
$$

with

$$
\left\langle\varphi^{\otimes N}, H_{N} \varphi^{\otimes N}\right\rangle=N\left\langle\varphi,\left(-\Delta+V^{\mathrm{ext}}+\frac{1}{2} v * \varphi^{2}\right) \varphi\right\rangle=: N e_{\mathrm{H}}
$$

For corresponding results in more singular scaling limits, see [40, 37, 38, 54, 4, 7, 52, 1] and [43, 41, $42,66,22,26,70,16,15,23]$.

The error in equation (1.3) is caused by $\mathcal{O}(1)$ particles which are excited from the condensate. To compute their energy, one decomposes $\Psi_{N}^{(n)}$ into contributions from condensate and excitations, as was first proposed in [36]. The excitations form a vector in a truncated Fock space over the orthogonal complement of $\varphi$, and the relation between $\Psi_{N}^{(n)}$ and the corresponding excitation vector is given by a unitary map

$$
U_{N, \varphi}: \mathfrak{H}^{N} \rightarrow \mathcal{F}_{\perp}^{\leq N}:=\bigoplus_{k=0}^{N} \bigotimes_{\text {sym }}^{k}\{\varphi\}^{\perp}, \quad \Psi_{N} \mapsto U_{N, \varphi} \Psi_{N}^{(n)}=: \chi_{\leq N}^{(n)}
$$

with the usual notation $\{\varphi\}^{\perp}:=\left\{\phi \in \mathfrak{H}:\langle\phi, \varphi\rangle_{\mathfrak{H}}=0\right\}$. Hence,

$$
\mathscr{E}_{N}^{(n)}=N e_{\mathrm{H}}+\left\langle U_{N, \varphi} \Psi_{N}^{(n)}, \mathbb{H}_{\leq N} U_{N, \varphi} \Psi_{N}^{(n)}\right\rangle_{\mathcal{F} \leq N}
$$

where

$$
\mathbb{H}_{\leq N}:=U_{N, \varphi}\left(H_{N}-N e_{\mathrm{H}}\right) U_{N, \varphi}^{*}: \mathcal{F}_{\perp}^{\leq N} \rightarrow \mathcal{F}_{\perp}^{\leq N}
$$

describes the energy due to excitations from the condensate.

By construction, the excitation Hamiltonian $\mathbb{H}_{\leq N}$ is explicitly $N$-dependent. To extract the contributions to the energy to each order in $\lambda_{N}$, we extend $\mathbb{H}_{\leq N}$ trivially to an operator $\mathbb{H}$ acting on the full excitation Fock space $\mathcal{F}_{\perp}$ and expand it formally as

$$
\mathbb{H}=\mathbb{H}_{0}+\sum_{j \geq 1} \lambda_{N}^{\frac{j}{2}} \mathbb{H}_{j}
$$

The coefficients $\mathbb{H}_{j}$ are $N$-independent operators on $\mathcal{F}_{\perp}$, which are explicitly given in terms of $\varphi$ and $v$ (see Definitions 2.4 and 3.3). In particular, $\mathbb{H}_{j}$ contains an even number of creation/annihilation operators for $j$ even, and an odd number for $j$ odd. 
The leading order term $\mathbb{H}_{0}$ is the well-known Bogoliubov Hamiltonian, which was first proposed by Bogoliubov in 1947 [9]. It is quadratic in the number of creation/annihilation operators and can be diagonalised by Bogoliubov transformations. The spectrum of $\mathbb{H}_{0}$ gives the $\mathcal{O}(1)$ contribution in equation (1.2) - that is, for any $v \in \mathbb{N}_{0}$, there exists an eigenvalue $E_{0}^{(n)}$ of $\mathbb{H}_{0}$ such that

$$
\lim _{N \rightarrow \infty}\left(\mathscr{E}_{N}^{(v)}-N e_{\mathrm{H}}\right)=E_{0}^{(n)},
$$

with

$$
E_{0}^{(0)}<E_{0}^{(1)}<\cdots<E_{0}^{(n)}<\cdots
$$

the eigenvalues of $\mathbb{H}_{0}$. For bounded interactions $v$, this was shown in [65] for the homogeneous setting and in [27] for the inhomogeneous case. Lewin, Nam, Serfaty and Solovej [36] proved equation (1.9) for a larger class of models, including a class of unbounded interaction potentials as well as a variety of one-particle operators. Moreover, related results on the torus were obtained in [44, 48]. All error estimates proven in $[65,27,36,44]$ are at best of the order $\mathcal{O}\left(N^{-1 / 2}\right)$. We refer to $[21,55,6,5]$ for similar results in more singular scaling limits.

In this paper, we derive the remaining terms in the expansion (1.2). To keep the notation simple, we restrict - for the remainder of this introduction - to the (nondegenerate) ground state. Formally, the coefficients in equation (1.2) can be determined by Rayleigh-Schrödinger perturbation theory in the small parameter $\lambda_{N}^{1 / 2}$. Let us denote by $\chi_{0}$ the (nondegenerate) normalised ground state of $\mathbb{H}_{0}$, and by $\mathbb{P}_{0}$ and $\mathbb{Q}_{0}$ the corresponding orthogonal projections on $\mathcal{F}_{\perp}-$ that is,

$$
\mathbb{H}_{0} \chi_{0}=E_{0}^{(0)} \chi_{0}, \quad \mathbb{P}_{0}=\left|\chi_{0}\right\rangle\left\langle\chi_{0}\right|, \quad \mathbb{Q}_{0}=\mathbb{1}-\mathbb{P}_{0} .
$$

By equation (1.8), the first-order perturbation of $\mathbb{H}_{0}$ is

$$
\mathbb{H}=\mathbb{H}_{0}+\lambda_{N}^{\frac{1}{2}} \mathbb{H}_{1}+\mathcal{O}\left(\lambda_{N}\right),
$$

and hence first-order perturbation theory yields (see, e.g., [63, Chapter 5])

$$
\mathscr{E}_{N}^{(0)}-N e_{\mathrm{H}}=E_{0}^{(0)}+\lambda_{N}^{\frac{1}{2}}\left\langle\chi_{0}, \mathbb{H}_{1} \chi_{0}\right\rangle_{\mathcal{F}_{\perp}}+\mathcal{O}\left(\lambda_{N}\right)=E_{0}^{(0)}+\mathcal{O}\left(\lambda_{N}\right)
$$

Here, the $\mathcal{O}\left(\lambda_{N}^{1 / 2}\right)$ contribution vanishes by Wick's rule because $\mathbb{H}_{1}$ contains an odd number of creation/annihilation operators and $\chi_{0}$ is quasi-free. For the next order, second-order perturbation theory for the Hamiltonian

$$
\mathbb{H}=\mathbb{H}_{0}+\lambda_{N}^{\frac{1}{2}} \mathbb{H}_{1}+\lambda_{N} \mathbb{H}_{2}+\mathcal{O}\left(\lambda_{N}^{3 / 2}\right)
$$

yields

$$
\mathscr{E}_{N}^{(0)}-N e_{\mathrm{H}}=E_{0}^{(0)}+\lambda_{N}\left\langle\chi_{0},\left(\mathbb{H}_{2}+\mathbb{H}_{1} \frac{\mathbb{Q}_{0}}{E_{0}^{(0)}-\mathbb{H}_{0}} \mathbb{H}_{1}\right) \chi_{0}\right\rangle_{\mathcal{F}_{\perp}}+\mathcal{O}\left(\lambda_{N}^{2}\right),
$$

and the higher orders are constructed similarly. In particular, all terms in the expansion corresponding to half-integer powers of $\lambda_{N}$ vanish.

In our main result, we make this formal argument rigorous by proving an asymptotic expansion for the ground-state projector $\mathbb{P}$ of $\mathbb{H}$. Recall that

$$
\mathbb{P}=\frac{1}{2 \pi \mathrm{i}} \oint_{\gamma} \frac{1}{z-\mathbb{H}} \mathrm{d} z, \quad \mathbb{P}_{0}=\frac{1}{2 \pi \mathrm{i}} \oint_{\gamma} \frac{1}{z-\mathbb{H}_{0}} \mathrm{~d} z,
$$


for some closed contour $\gamma$ which encloses both $\mathscr{E}_{N}^{(0)}-N e_{\mathrm{H}}$ and $E_{0}^{(0)}$ and leaves the remaining spectra of $\mathbb{H}$ and $\mathbb{H}_{0}$ outside. The existence of such a contour with length of order 1 is, for sufficiently large $N$, guaranteed by equation (1.9). Using equation (1.8), we expand the resolvent of $\mathbb{H}$ around the resolvent of $\mathbb{H}_{0}$, which results in an expansion of $\mathbb{P}$, and the trace against $\mathbb{H}$ recovers equations (1.12) and (1.14) (see Theorem 2). Finally, we show that the error is subleading with respect to the order of the approximation.

In fact, we prove a more general statement, which can be understood as asymptotic expansion of the ground state of $H_{N}$ : for any operator $A^{(m)}$ on $\mathfrak{S}^{m}$ that is relatively bounded with respect to $\sum_{j=1}^{m}\left(-\Delta_{j}+V^{\mathrm{ext}}\left(x_{j}\right)\right)$, it holds that

$$
\operatorname{Tr}_{\mathfrak{Y}^{N}} \mathcal{A}_{N}^{(m)} P_{N}=\operatorname{Tr}_{\mathcal{F}_{\perp}} \mathbb{A}_{N}^{(m)} \mathbb{P}_{0}+\sum_{\ell=1}^{a} \lambda_{N}^{\frac{\ell}{2}} \operatorname{Tr}_{\mathcal{F}_{\perp}} \mathbb{A}_{N}^{(m)} \mathbb{P}_{\ell}+\mathcal{O}\left(\lambda_{N}^{\frac{a+2}{2}}\right),
$$

where $P_{N}$ denotes the projector onto the ground state of $H_{N}, \mathcal{A}_{N}^{(m)}$ is the symmetrised version of $A^{(m)}$ on $\mathfrak{H}^{N}, \mathbb{A}_{N}^{(m)}$ denotes the conjugation of $\mathcal{A}_{N}^{(m)}$ with $U_{N, \varphi}$, and $\mathbb{P}_{\ell}$ is the $\ell$ th order in the expansion of the projector $\mathbb{P}$. The full statement, which extends to excited states with energies of order 1 above the ground state, is given in Theorem 1.

Our analysis is restricted to the mean-field regime. It is an open question whether a similar statement holds true for interaction potentials that converge to a delta distribution as $N \rightarrow \infty$.

In the physics literature, higher-order corrections to the Lee-Huang-Yang formula for the groundstate energy of a low-density Bose gas with short-range interactions were studied in the 1950s in [17, $18,2,3,69]$, and a series expansion for the ground-state energy was conjectured in [64, 32]. We refer to $[13,12,68]$ for more recent contributions. However, to the best of our knowledge, the rigorous derivation of higher-order corrections to the Bogoliubov energy in the mean-field scaling has not been studied before. Other approaches to perturbations around Bogoliubov theory are based on the ideas of renormalisation groups and constructive field theory, which is very different from our rather direct approach. We refer to [19] for recent results and a review of the literature, which mostly treats more singular scalings than the mean-field regime.

Another approach was proposed by Pizzo in [59, 60, 61], where he considers a Bose gas on a torus in the mean-field regime. He constructs an expansion for the ground state and a fixed-point equation for the ground-state energy, first for a simpler three-mode Bogoliubov Hamiltonian [59] and subsequently, building on these results, for a Bogoliubov Hamiltonian [60] and the full Hamiltonian [61]. The main result is norm convergence of the expansion to the ground state to arbitrary precision. This expansion is based on a multiscale analysis in the number of excitations around a product state using Feshbach maps. In contrast to our work, this is done in the $N$-particle space, whereas we make use of the $N$-dependent unitary map $U_{N, \varphi}$ to work in the excitation Fock space $\mathcal{F}_{\perp}$.

Finally, we remark that our work is inspired by [11], where an analogous expansion of the dynamics generated by $H_{N}$ was constructed. Related results for the mean-field dynamics in Fock space have been obtained in [25, 24], and different approaches characterising the dynamics to any order in $1 / N$ are discussed in $[57,10]$. We also note that there are many recent results on the derivation of the Bogoliubov dynamics in the mean-field regime [30, 31, 35, 45], as well as in more singular scaling limits [28, 8, 49, 29, 33, 50, 20, 14, 58].

\section{Notation}

- We denote by $\mathfrak{C}$ an expression which may depend on constants fixed by the model - that is, constants whose values depend on $h$ and $\mathbb{H}_{0}$, such as norms of the Hartree minimiser $\varphi$, the gap $g_{\mathrm{H}}$ above the ground state of $h$ and norms $\left\|U_{0}\right\|_{\mathrm{op}}$ (the operator norm) and $\left\|V_{0}\right\|_{\mathrm{HS}}$ (the HilbertSchmidt norm) of the Bogoliubov transformation diagonalising $\mathbb{H}_{0}$. The notation $\mathfrak{C}(n)$ indicates that the constant may also depend on the number $n$ of the corresponding eigenvalue of $\mathbb{H}_{0}$, such as 
$\left|E_{0}^{(n)}\right|$, its degeneracy $\delta_{0}^{(n)}$ and the spectral gap above it. Finally, $\mathfrak{C}(n, a)$ implies the dependence on an additional parameter $a$. Constants may vary from line to line.

- Eigenvalues are always counted without multiplicity - that is, the (discrete) spectrum of an operator $T$ is denoted as $t^{(0)}<t^{(1)}<t^{(2)}<\cdots$, where each eigenvalue $t^{(j)}$ has some finite multiplicity $\delta^{(j)} \geq 1$.

○ We denote by $\boldsymbol{j}:=\left(j_{1}, \ldots, j_{n}\right)$ a multi-index and define $|\boldsymbol{j}|:=j_{1}+\cdots+j_{n}$. Moreover, we abbreviate

$$
x^{(k)}:=\left(x_{1}, \ldots, x_{k}\right), \quad \mathrm{d} x^{(k)}:=\mathrm{d} x_{1} \cdots \mathrm{d} x_{k}
$$

for $k \geq 1$ and $x_{j} \in \mathbb{R}^{d}$.

\section{Preliminaries}

\subsection{Assumptions}

We make the following assumptions on the external potential $V^{\text {ext }}$ and the interaction $v$ :

Assumption 1. Let $V^{\text {ext }}: \mathbb{R}^{d} \rightarrow \mathbb{R}$ be measurable, locally bounded and nonnegative, and let $V^{\text {ext }}(x)$ tend to infinity as $|x| \rightarrow \infty$, that is,

$$
\inf _{|x|>R} V^{e x t}(x) \rightarrow \infty \quad \text { as } \quad R \rightarrow \infty
$$

Assumption 1 implies that $V^{\text {ext }}$ must be a confining potential. It is, for example, satisfied by $V^{\text {ext }}(x)=$ $\omega x^{2}$ for $\omega>0$. Let us introduce the abbreviation

$$
T: \mathfrak{H} \supset \mathcal{D}(T) \rightarrow \mathfrak{H}, \quad T:=-\Delta+V^{\text {ext }} .
$$

We denote by

$$
T_{j}:=\mathbb{1} \otimes \cdots \otimes \mathbb{1} \otimes T \otimes \mathbb{1} \otimes \cdots \otimes \mathbb{1}
$$

the operator acting as $T$ on the $j$ th coordinate.

Assumption 2. Let $v: \mathbb{R}^{d} \rightarrow \mathbb{R}$ be measurable with $v(-x)=v(x)$ and $v \not \equiv 0$, and assume that there exists a constant $C>0$ such that, in the sense of operators on $Q(-\Delta)=H^{1}\left(\mathbb{R}^{d}\right)$,

$$
|v|^{2} \leq C(1-\Delta) \text {. }
$$

In addition, assume that $v$ is of positive type - that is, that it has a nonnegative Fourier transform.

Assumption 2 is clearly satisfied by any bounded potential with positive Fourier transform. Moreover, by Hardy's inequality, it is fulfilled by the repulsive Coulomb potential in $d=3$ dimensions.

Remark 2.1. (a) Note that inequality (2.3) implies that

$$
2\left|v\left(x_{1}-x_{2}\right)\right| \leq 1+\left|v\left(x_{1}-x_{2}\right)\right|^{2} \leq \mathfrak{C}\left(-\Delta_{1}-\Delta_{2}+1\right) \leq \mathfrak{C}\left(T_{1}+T_{2}+1\right)
$$

in the sense of operators on $\mathcal{Q}\left(T_{1}+T_{2}\right) \subset \mathfrak{H}^{2}$, because $V^{\text {ext }} \geq 0$. In particular,

$$
\begin{aligned}
\left\|v * \phi^{2}\right\|_{\infty} & \leq \mathfrak{C}\left(\|\nabla \phi\|^{2}+1\right), \\
\left\langle\phi \otimes \phi,|v(x-y)|^{2} \phi \otimes \phi\right\rangle_{\mathfrak{S}^{2}} & \leq \mathfrak{C}\langle\phi,(T+1) \phi\rangle
\end{aligned}
$$

for any normalised $\phi \in \mathcal{Q}(T)$. Moreover, $v$ being of positive type implies that

$$
\int_{\mathbb{R}^{2 d}} \mathrm{~d} x \mathrm{~d} y \overline{\phi(x)} v(x-y) \phi(y) \geq 0 .
$$


(b) Assumptions 1 and 2 imply that $|v|^{2} \leq \varepsilon T^{2}+C^{2} \varepsilon^{-1}+C$ for any $\varepsilon>0$, hence $H_{N}$ is (for each $N$ ) self-adjoint on its domain $\mathcal{D}\left(\sum_{j=1}^{N} T_{j}\right)$ by the Kato-Rellich theorem.

(c) Since $V^{\text {ext }}$ is measurable and locally bounded and tends to infinity, it is bounded below, and we take its lower bound to be 0 only for convenience.

Next, we recall the Hartree energy functional, which is defined as

$$
\mathcal{E}_{\mathrm{H}}[\phi]:=\int_{\mathbb{R}^{d}}\left(|\nabla \phi(x)|^{2}+V^{\mathrm{ext}}(x)|\phi(x)|^{2}\right) \mathrm{d} x+\frac{1}{2} \int_{\mathbb{R}^{2 d}} v(x-y)|\phi(x)|^{2}|\phi(y)|^{2} \mathrm{~d} x \mathrm{~d} y
$$

for $\phi \in \mathcal{D}_{\mathrm{H}}$, with

$$
\mathcal{D}_{\mathrm{H}}:=\left\{\phi \in \mathcal{Q}(T):\|\phi\|_{\mathfrak{H}}=1\right\} \subset \mathfrak{H}
$$

Its infimum is denoted by

$$
e_{\mathrm{H}}:=\inf _{\phi \in \mathcal{D}_{\mathrm{H}}} \mathcal{E}_{\mathrm{H}}[\phi]
$$

Under Assumptions 1 and 2, $\mathcal{E}_{\mathrm{H}}$ admits a unique, strictly positive minimiser $\varphi$, which solves the stationary Hartree equation:

Lemma 2.2. Let Assumptions 1 and 2 hold.

(a) There exists a unique (up to a phase) $\varphi \in \mathcal{D}_{H}$ such that

$$
\mathcal{E}_{H}[\varphi]=e_{H},
$$

and we choose $\varphi$ strictly positive. The minimiser $\varphi$ solves the stationary Hartree equation,

$$
h \varphi=0
$$

in the sense of distributions, where

$$
h: \mathfrak{H} \supset \mathcal{D}(T) \rightarrow \mathfrak{H}, \quad h: T+v * \varphi^{2}-\mu_{H},
$$

with Lagrange multiplier $\mu_{H} \in \mathbb{R}$ given by

$$
\mu_{H}:=\left\langle\varphi,\left(T+v * \varphi^{2}\right) \varphi\right\rangle
$$

(b) The operator $h$ is self-adjoint on its domain $\mathcal{D}(T)$ and its spectrum is purely discrete. The minimiser $\varphi$ of $\mathcal{E}_{H}$ is the unique ground state $\varphi$ of $h$, and there exists a complete set of normalised eigenfunctions $\left\{\varphi_{j}\right\}_{j \geq 0}$ for $h$. The spectrum and eigenstates of $h$ are denoted as

$$
h \varphi_{j}=\varepsilon^{(j)} \varphi_{j}, \quad 0=\varepsilon^{(0)}<\varepsilon^{(1)}<\cdots, \quad \varphi_{0}:=\varphi .
$$

In particular, the spectral gap $g_{H}$ above the ground state of $h$ is positive:

$$
g_{H}:=\varepsilon^{(1)}-\varepsilon^{(0)}=\varepsilon^{(1)}>0 .
$$

(c) Define $K: \mathfrak{H} \rightarrow \mathfrak{G}$ as the operator with kernel

$$
K(x ; y):=v(x-y) \varphi(x) \varphi(y) .
$$


Then $K$ is positive and Hilbert-Schmidt. Moreover,

$$
\mathcal{A}:=\left(\begin{array}{cc}
h+q K q & q K q \\
q K q & h+q K q
\end{array}\right) \geq g_{H}>0 \text { on } \mathfrak{H}_{\perp} \oplus \mathfrak{H}_{\perp}
$$

for $\mathfrak{H}_{\perp}:=\{\varphi\}^{\perp}$ and where $q$ denotes the orthogonal projection onto $\mathfrak{H}_{\perp}$-that is,

$$
p:=|\varphi\rangle\langle\varphi|, \quad q:=\mathbb{1}_{\mathfrak{Y}}-p .
$$

Proof. For part (a), note first that $\mathcal{E}_{\mathrm{H}} \geq 0$ on $\mathcal{D}_{\mathrm{H}}$, hence there exists a sequence $\left\{\phi_{n}\right\}_{n} \subset \mathcal{D}_{\mathrm{H}}$ such that $\mathcal{E}_{\mathrm{H}}\left[\phi_{n}\right] \rightarrow e_{\mathrm{H}}$. Moreover, $\left\langle\phi_{n}, T \phi_{n}\right\rangle \leq C$, because $D\left(\left|\phi_{n}\right|^{2},\left|\phi_{n}\right|^{2}\right) \geq 0$ by inequality (2.7), where $D(f, g):=\frac{1}{2} \int_{\mathbb{R}^{2 d}} \mathrm{~d} x \mathrm{~d} y \overline{f(x)} v(x-y) g(y)$. Since $T$ has a compact resolvent by Assumption 1 , $\mathcal{D}_{C}:=\{\psi \in \mathcal{Q}(T):\|\psi\| \leq 1,\langle\psi, T \psi\rangle \leq C\}$ is compact [62, Theorems XIII.16 and XIII.64], and there exists a subsequence such that $\phi_{n} \rightarrow \phi \in \mathcal{D}_{C}$ strongly in $\mathfrak{H}$. For $\varrho:=|\phi|^{2}$ and $\varrho_{n}:=\left|\phi_{n}\right|^{2}$, $\|\varrho * v\|_{\infty} \leq C$ by the bound (2.5) and $\int \rho_{n} \rightarrow \int \rho$, hence

$$
\lim _{n \rightarrow \infty} D\left(\varrho_{n}, \varrho_{n}\right) \geq 2 \lim _{n \rightarrow \infty} D\left(\varrho_{n}-\varrho, \varrho\right)+D(\varrho, \varrho)=D(\varrho, \varrho)
$$

Since $\mathcal{D}_{C}$ is weakly compact in both $H^{1}\left(\mathbb{R}^{d}\right)$ and the $L^{2}$-space with norm $\|\psi\|_{V}^{2}:=\int V^{\text {ext }}|\psi|^{2}$, we find, passing again to a subsequence, that $\lim _{n \rightarrow \infty}\left\langle\phi_{n}, T \phi_{n}\right\rangle \geq\langle\phi, T \phi\rangle$ by weak lower semicontinuity of both norms. With this, part (a) can be shown as in [40, Lemmas A.1-4]. We denote the unique strictly positive minimiser by $\varphi$.

Part (b) is a consequence of the estimate (2.5) and Assumption 1, by Kato-Rellich and [62, Theorems XIII.16 and XIII.64]. Finally, the first part of (c) is implied by the bound (2.6), and the second part follows since $K \geq 0$ by inequality (2.7) and $h \geq g_{\mathrm{H}}$ on $\mathfrak{H}_{\perp}$ by part (b).

In summary, Assumptions 1 and 2 provide all the necessary properties of the effective one-body operator $h$, in particular the existence of a finite spectral gap above the ground state. In addition, we require the Hartree functional to be a valid description for the $N$-body energy as $N \rightarrow \infty$. Put differently, we assume that $N$-body states with an energy of order 1 above the ground state exhibit complete BoseEinstein condensation in the Hartree minimiser $\varphi$. This is implied by the following statement:

Assumption 3. Assume that there exist constants $C_{1} \geq 0$ and $0<C_{2} \leq 1$, as well as a function $\varepsilon: \mathbb{N} \rightarrow \mathbb{R}_{0}^{+}$with

$$
\lim _{N \rightarrow \infty} N^{-\frac{1}{3}} \varepsilon(N) \leq C_{1},
$$

such that

$$
H_{N}-N e_{H} \geq C_{2} \sum_{j=1}^{N} h_{j}-\varepsilon(N)
$$

in the sense of operators on $\mathcal{D}\left(H_{N}\right)$.

We do not know how to prove inequality (2.20) under our generic Assumptions 1 and 2. However, it is known to be true for the examples we have in mind: any bounded and positive definite interaction potential $v$ satisfies Assumption 3 with optimal rate $\varepsilon(N)=\mathcal{O}(1)$ [27, Lemma 1 and Remark 2]. Moreover, the repulsive three-dimensional Coulomb potential fulfils Assumption 3 with $\varepsilon(N)=\mathcal{O}\left(N^{1 / 3}\right)$ [36, Lemma 3.1].

\subsection{Excitation Fock space and excitation Hamiltonian}

In this section, we review the excitation map $U_{N, \varphi}$ from definition (1.5), which was introduced in [36] and maps an $N$-body wave function to the corresponding excitation vector. Recall that any $\Psi \in \mathfrak{H}_{\text {sym }}^{N}$ 
can be decomposed into condensate and excitations as

$$
\Psi=\sum_{k=0}^{N} \varphi^{\otimes(N-k)} \otimes_{s} \chi^{(k)}, \quad \chi^{(k)} \in \bigotimes_{\text {sym }}^{k} \mathfrak{H}_{\perp}
$$

with $\otimes_{S}$ the symmetric tensor product, which is for $\psi_{a} \in \mathfrak{H}^{a}$ and $\psi_{b} \in \mathfrak{H}^{b}$ defined as

$$
\begin{aligned}
&\left(\psi_{a} \otimes_{s} \psi_{b}\right)\left(x_{1}, \ldots, x_{a+b}\right):= \\
& \frac{1}{\sqrt{a ! b !(a+b) !}} \sum_{\sigma \in \mathfrak{S}_{a+b}} \psi_{a}\left(x_{\sigma(1)}, \ldots, x_{\sigma(a)}\right) \psi_{b}\left(x_{\sigma(a+1)}, \ldots, x_{\sigma(a+b)}\right),
\end{aligned}
$$

with $\mathfrak{S}_{a+b}$ the set of all permutations of $a+b$ elements. The sequence

$$
\chi_{\leq N}:=\left(\chi^{(k)}\right)_{k=0}^{N}
$$

of $k$-particle excitations forms a vector in the truncated excitation Fock space over $\mathfrak{H}_{\perp}$,

$$
\mathcal{F}_{\perp}^{\leq N}=\bigoplus_{k=0}^{N} \bigotimes_{\mathrm{sym}}^{k} \mathfrak{H}_{\perp} \subset \mathcal{F}_{\perp}=\bigoplus_{k=0}^{\infty} \bigotimes_{\mathrm{sym}}^{k} \mathfrak{H}_{\perp}
$$

and vectors in $\mathcal{F}_{\perp}$ are denoted as

$$
\boldsymbol{\phi}=\left(\phi^{(0)}, \phi^{(1)}, \ldots, \phi^{(k)}, \ldots\right), \quad \phi_{\leq N}=\left(\phi^{(0)}, \phi^{(1)}, \ldots, \phi^{(N)}\right) .
$$

We consider the decomposition of $\mathcal{F}_{\perp}$ into the subspaces

$$
\mathcal{F}_{\perp}=\mathcal{F}_{\perp}^{\leq N} \oplus \mathcal{F}_{\perp}^{>N}
$$

and in the following all direct sums are understood with respect to this decomposition. The creation and annihilation operators on $\mathcal{F}_{\perp}$ are

$$
\left(a^{\dagger}(f) \phi\right)^{(k)}\left(x_{1}, \ldots, x_{k}\right)=\frac{1}{\sqrt{k}} \sum_{j=1}^{k} f\left(x_{j}\right) \phi^{(k-1)}\left(x_{1}, \ldots, x_{j-1}, x_{j+1}, \ldots, x_{k}\right)
$$

for $k \geq 1$ and

$$
(a(f) \phi)^{(k)}\left(x_{1}, \ldots, x_{k}\right)=\sqrt{k+1} \int \mathrm{d} x \overline{f(x)} \phi^{(k+1)}\left(x_{1}, \ldots, x_{k}, x\right)
$$

for $k \geq 0$, where $f \in \mathfrak{H}_{\perp}$ and $\phi \in \mathcal{F}_{\perp}$. They can be expressed in terms of the operator-valued distributions $a_{x}^{\dagger}$ and $a_{x}$,

$$
a^{\dagger}(f)=\int \mathrm{d} x f(x) a_{x}^{\dagger}, \quad a(f)=\int \mathrm{d} x \overline{f(x)} a_{x},
$$

which satisfy the canonical commutation relations

$$
\left[a_{x}, a_{y}^{\dagger}\right]=\delta(x-y), \quad\left[a_{x}, a_{y}\right]=\left[a_{x}^{\dagger}, a_{y}^{\dagger}\right]=0 .
$$


We denote the second quantisation in $\mathcal{F}_{\perp}$ of an $m$-body operator $T^{(m)}$ by

$$
\begin{aligned}
\mathrm{d} \Gamma_{\perp} & \left(T^{(m)}\right) \\
= & 0 \oplus \cdots \oplus 0 \oplus \bigoplus_{k \geq 0} \sum_{\substack{1 \leq j_{1}<\cdots<j_{m} \leq m+k \\
=}} T_{j_{1}, \ldots, j_{m}}^{(m)} \sum_{\substack{i_{1}, \ldots, i_{m} \geq 1 \\
j_{1}, \ldots, j_{m} \geq 1}}\left\langle\psi_{i_{1}} \otimes \cdots \otimes \psi_{i_{m}}, T^{(m)} \psi_{j_{1}} \otimes \cdots \otimes \psi_{j_{m}}\right\rangle \\
& \times a^{\dagger}\left(\psi_{i_{1}}\right) \cdots a^{\dagger}\left(\psi_{i_{m}}\right) a\left(\psi_{j_{1}}\right) \cdots a\left(\psi_{j_{m}}\right)
\end{aligned}
$$

for any orthonormal basis $\left\{\psi_{j}\right\}_{j \geq 1}$ of $\mathfrak{H}_{\perp}$. Equivalently,

$$
\mathrm{d} \Gamma_{\perp}\left(T^{(m)}\right)=\mathrm{d} \Gamma_{\perp}\left(q^{\otimes m} T^{(m)} q^{\otimes m}\right)=\mathrm{d} \Gamma\left(q^{\otimes m} T^{(m)} q^{\otimes m}\right),
$$

where $\mathrm{d} \Gamma$ denotes the usual second quantisation in the Fock space over the full space $\mathfrak{H}$. Finally, the number operator on $\mathcal{F}_{\perp}$ is given by

$$
\mathcal{N}_{\perp}:=\mathrm{d} \Gamma_{\perp}(\mathbb{1})=\mathrm{d} \Gamma_{\perp}(q), \quad\left(\mathcal{N}_{\perp} \phi\right)^{(k)}=k \phi^{(k)} \text { for } \phi \in \mathcal{F}_{\perp} .
$$

An $N$-body state $\Psi$ is mapped onto its corresponding excitation vector $\chi_{\leq N}$ by

$$
U_{N, \varphi}: \mathfrak{H}^{N} \rightarrow \mathcal{F}_{\perp}^{\leq N}, \quad \Psi \mapsto U_{N, \varphi} \Psi:=\chi_{\leq N},
$$

which is unitary and acts as

$$
U_{N, \varphi} \Psi=\bigoplus_{j=0}^{N} q^{\otimes j}\left(\frac{a(\varphi)^{N-j}}{\sqrt{(N-j) !}} \Psi\right) \text { for } \Psi \in \mathfrak{H}^{N}
$$

by [36, Proposition 4.2]. Note that the product state $\varphi^{\otimes N}$ is mapped to the vacuum of $\mathcal{F}_{\perp}^{\leq N}$,

$$
U_{N, \varphi} \varphi^{\otimes N}=(1,0,0, \ldots, 0)=:|\Omega\rangle .
$$

For $f, g \in \mathfrak{H}_{\perp}$, equation (2.35) yields the substitution rules

$$
\begin{aligned}
& U_{N, \varphi} a^{\dagger}(\varphi) a(\varphi) U_{N, \varphi}^{*}=N-\mathcal{N}_{\perp}, \\
& U_{N, \varphi} a^{\dagger}(f) a(\varphi) U_{N, \varphi}^{*}=a^{\dagger}(f) \sqrt{N-\mathcal{N}_{\perp},} \\
& U_{N, \varphi} a^{\dagger}(\varphi) a(g) U_{N, \varphi}^{*}=\sqrt{N-\mathcal{N}_{\perp}} a(g), \\
& U_{N, \varphi} a^{\dagger}(f) a(g) U_{N, \varphi}^{*}=a^{\dagger}(f) a(g)
\end{aligned}
$$

as identities on $\mathcal{F}_{\perp}^{\leq N}$. As explained in the introduction, conjugating $H_{N}$ with $U_{N, \varphi}$ extracts the contribution to the energy which is due to excitations from the condensate.

Definition 2.3. Define

$$
\mathbb{H}_{\leq N}:=U_{N, \varphi}\left(H_{N}-N e_{\mathrm{H}}\right) U_{N, \varphi}^{*}
$$

as an operator on $\mathcal{F}_{\perp}^{\leq N}$. The eigenvalues $E^{(n)}$ of $\mathbb{H}_{\leq N}$ relate to the eigenvalues $\mathscr{E}_{N}^{(n)}$ of $H_{N}$ as

$$
E^{(n)}=\mathscr{E}_{N}^{(n)}-N e_{\mathrm{H}}, \quad n \in \mathbb{N}_{0}
$$


As a consequence of the substitution rules (2.37), $\mathbb{H}_{\leq N}$ can be expressed as

$$
\begin{aligned}
\mathbb{H}_{\leq N}= & \mathbb{K}_{0}+\left(\frac{N-\mathcal{N}_{\perp}}{N-1}\right) \mathbb{K}_{1} \\
& +\left(\mathbb{K}_{2} \frac{\sqrt{\left(N-\mathcal{N}_{\perp}\right)\left(N-\mathcal{N}_{\perp}-1\right)}}{N-1}+\frac{\sqrt{\left(N-\mathcal{N}_{\perp}\right)\left(N-\mathcal{N}_{\perp}-1\right)}}{N-1} \mathbb{K}_{2}^{*}\right) \\
& +\left(\mathbb{K}_{3} \frac{\sqrt{N-\mathcal{N}_{\perp}}}{N-1}+\frac{\sqrt{N-\mathcal{N}_{\perp}}}{N-1} \mathbb{K}_{3}^{*}\right)+\frac{1}{N-1} \mathbb{K}_{4},
\end{aligned}
$$

where we used the shorthand notation

$$
\begin{aligned}
& \mathbb{K}_{0}:=\int \mathrm{d} x a_{x}^{\dagger} h_{x} a_{x}, \\
& \mathbb{K}_{1}:=\int \mathrm{d} x_{1} \mathrm{~d} x_{2} K_{1}\left(x_{1} ; x_{2}\right) a_{x_{1}}^{\dagger} a_{x_{2}}, \\
& \mathbb{K}_{2}:=\frac{1}{2} \int \mathrm{d} x_{1} \mathrm{~d} x_{2} K_{2}\left(x_{1}, x_{2}\right) a_{x_{1}}^{\dagger} a_{x_{2}}^{\dagger}, \\
& \mathbb{K}_{3}:=\int \mathrm{d} x^{(3)} K_{3}\left(x_{1}, x_{2} ; x_{3}\right) a_{x_{1}}^{\dagger} a_{x_{2}}^{\dagger} a_{x_{3}} \\
& \mathbb{K}_{4}:=\frac{1}{2} \int \mathrm{d} x^{(4)} K_{4}\left(x_{1}, x_{2} ; x_{3}, x_{4}\right) a_{x_{1}}^{\dagger} a_{x_{2}}^{\dagger} a_{x_{3}} a_{x_{4}},
\end{aligned}
$$

with

$$
\begin{aligned}
K_{1}: \mathfrak{H}_{\perp} & \rightarrow \mathfrak{H}_{\perp}, \quad K_{1}:=q K q, \\
K_{2} \in \mathfrak{H}_{\perp} & \otimes \mathfrak{H}_{\perp}, \quad K_{2}\left(x_{1}, x_{2}\right):=\left(q_{1} q_{2} K\right)\left(x_{1}, x_{2}\right), \\
K_{3}: \mathfrak{H}_{\perp} & \rightarrow \mathfrak{H}_{\perp} \otimes \mathfrak{H}_{\perp}, \\
\psi & \mapsto\left(K_{3} \psi\right)\left(x_{1}, x_{2}\right):=q_{1} q_{2} W\left(x_{1}, x_{2}\right) \varphi\left(x_{1}\right)\left(q_{2} \psi\right)\left(x_{2}\right), \\
K_{3}^{*}: \mathfrak{H}_{\perp} & \otimes \mathfrak{H}_{\perp} \rightarrow \mathfrak{H}_{\perp}, \\
\psi & \mapsto\left(K_{3}^{*} \psi\right)\left(x_{1}\right)=q_{1} \int \mathrm{d} x_{2} \varphi\left(x_{2}\right) W\left(x_{1}, x_{2}\right)\left(q_{1} q_{2} \psi\right)\left(x_{1}, x_{2}\right), \\
K_{4}: \mathfrak{H}_{\perp} & \otimes \mathfrak{S}_{\perp} \rightarrow \mathfrak{H}_{\perp} \otimes \mathfrak{H}_{\perp}, \\
\psi & \mapsto\left(K_{4} \psi\right)\left(x_{1}, x_{2}\right):=q_{1} q_{2} W\left(x_{1}, x_{2}\right)\left(q_{1} q_{2} \psi\right)\left(x_{1}, x_{2}\right) .
\end{aligned}
$$

Here, $K\left(x_{1}, x_{2}\right)$ is defined as in definition (2.16), $K$ is the operator with kernel $K\left(x_{1}, x_{2}\right)$ and $W$ is the multiplication operator on $\mathfrak{H}_{\perp} \otimes \mathfrak{G}_{\perp}$ defined by

$$
W\left(x_{1}, x_{2}\right):=v\left(x_{1}-x_{2}\right)-\left(v * \varphi^{2}\right)\left(x_{1}\right)-\left(v * \varphi^{2}\right)\left(x_{2}\right)+\left\langle\varphi, v * \varphi^{2} \varphi\right\rangle .
$$

The notation is understood such that the projections $q_{1}, q_{2}$ act on the respective functions on their right. For example, the function $K_{3} \psi \in \mathfrak{S}_{\perp} \otimes \mathfrak{H}_{\perp}$ is obtained from $\psi \in \mathfrak{H}_{\perp}$ by taking the tensor product of $q \psi$ and $\varphi$, acting on it with the multiplication operator $W$ and finally projecting the resulting function onto the subspace $\mathfrak{S}_{\perp} \otimes \mathfrak{H}_{\perp}$. Note that $q \psi=\psi$ for $\psi \in \mathfrak{Y}_{\perp}$, hence the projection $q$ in front of $\psi$ is not necessary here, but it allows us to extend $K_{3}$ to a map on the full space $\mathfrak{h}$. An analogous observation applies to $K_{1}, K_{3}^{*}$ and $K_{4}$. An explicit formula for $\mathbb{H}_{\leq N}$ was first derived in [36, Section 4], and we rewrote it in a way that is more convenient for our analysis (see Appendix A).

Finally, we recall the Bogoliubov Hamiltonian $\mathbb{H}_{0}$ and introduce some notation: 
Definition 2.4. The Bogoliubov Hamiltonian $\mathbb{H}_{0}$ for the model (1.1) is defined as

$$
\mathbb{H}_{0}:=\mathbb{K}_{0}+\mathbb{K}_{1}+\mathbb{K}_{2}+\mathbb{K}_{2}^{*}
$$

with $\mathbb{K}_{j}$ as in definition (2.41). The eigenvalues of $\mathbb{H}_{0}$ are denoted as

$$
E_{0}^{(0)}<E_{0}^{(1)}<\cdots<E_{0}^{(n)}<\cdots,
$$

with associated eigenspaces

$$
\mathfrak{E}_{0}^{(n)}:=\left\{\phi \in \mathcal{F}_{\perp}: \mathbb{H}_{0} \phi=E_{0}^{(n)} \phi\right\}, \quad \delta_{0}^{(n)}:=\operatorname{dim} \mathfrak{E}_{0}^{(n)} .
$$

The spectral gap of $\mathbb{H}_{0}$ above $E_{0}^{(n)}$ is defined as

$$
g_{0}^{(n)}:=E_{0}^{(n+1)}-E_{0}^{(n)}, \quad n \in \mathbb{N}_{0},
$$

and the projections onto $\mathfrak{E}_{0}^{(n)}$ and its orthogonal complement are given by

$$
\mathbb{P}_{0}^{(n)}:=\mathbb{1}_{\mathfrak{E}_{0}^{(n)}}, \quad \mathbb{Q}_{0}^{(n)}:=\mathbb{1}_{\mathcal{F}_{\perp}}-\mathbb{P}_{0}^{(n)}
$$

We denote normalised elements of $\mathfrak{E}_{0}^{(n)}$ as $\chi_{0}^{(n)}$.

\section{Results}

\subsection{Main results}

Our goal is a perturbative expansion of the spectral projectors of $\mathbb{H}_{\leq N}=U_{N, \varphi}\left(H_{N}-N e_{\mathrm{H}}\right) U_{N, \varphi}^{*}$ around the spectral projectors of $\mathbb{H}_{0}$. For our analysis, it is crucial that the low-energy eigenvalues of $\mathbb{H}_{\leq N}$ converge to the corresponding eigenvalues of $\mathbb{H}_{0}$, and the same holds true (in a suitable sense) for the respective eigenstates. This was proven in $[65,27,36]$, and we collect the rigorous results in Lemma 4.8. If different eigenvalues of $H_{N}-N e_{\mathrm{H}}$ converge to the same limiting eigenvalue of $\mathbb{H}_{0}$ as $N \rightarrow \infty$, we consider the sum of all corresponding spectral projections of $H_{N}$ :

Definition 3.1. Define

$$
\iota^{(n)}:=\left\{v \in \mathbb{N}_{0}: \lim _{N \rightarrow \infty}\left(\mathscr{E}_{N}^{(v)}-N e_{\mathrm{H}}\right)=E_{0}^{(n)}\right\}
$$

and

$$
\mathfrak{E}_{N}^{(n)}:=\bigoplus_{\nu \in \iota^{(n)}} \widetilde{\mathfrak{E}}_{N}^{(\nu)},
$$

with

$$
\widetilde{\mathfrak{E}}_{N}^{(v)}:=\left\{\Psi \in \mathfrak{H}_{\mathrm{sym}}^{N}: H_{N} \Psi=\mathscr{E}_{N}^{(v)} \Psi\right\}, \quad \delta_{N}^{(v)}:=\operatorname{dim} \widetilde{\mathfrak{E}}_{N}^{(v)}
$$

The corresponding orthogonal projections are denoted by

$$
P_{N}^{(n)}:=\mathbb{1}_{\mathfrak{E}_{N}^{(n)}}
$$

By [36], the set $\iota^{(n)}$, which collects all eigenvalues of $H_{N}-N e_{\mathrm{H}}$ that converge to the eigenvalue $E_{0}^{(n)}$ of $\mathbb{H}_{0}$, is of the form $\{\ell, \ldots, \ell+j\}$ for some $\ell, j \geq 0$. Moreover, $1 \leq\left|\iota^{(n)}\right| \leq \delta_{0}^{(n)}$, where the second 
inequality is strict if at least one of the eigenvalues $\mathscr{E}_{N}^{(v)}$ is degenerate. The space $\mathfrak{E}_{N}^{(n)}$ is the direct sum of all eigenspaces of $H_{N}$ associated with eigenvalues with label $v \in \iota^{(n)}$, hence $\sum_{v \in \iota}(n) \delta_{N}^{(v)}=\delta_{0}^{(n)}$.

We consider expectation values with respect to $P_{N}^{(n)}$ for a natural class of $m$-body operators, namely for all operators that are relatively bounded with respect to $\sum_{j=1}^{m} T_{j}$. We use the following notation:

Definition 3.2. For $m \in \mathbb{N}$, let $A^{(m)}$ be some operator acting on $\mathfrak{S}^{m}$. We denote the corresponding symmetrised operator on $\mathfrak{H}^{N}$ by

$$
\mathcal{A}_{N}^{(m)}:=\left(\begin{array}{l}
N \\
m
\end{array}\right)^{-1} \sum_{1 \leq j_{1}<\cdots<j_{m} \leq N} A_{j_{1}, \ldots, j_{m}}^{(m)},
$$

where $A_{j_{1}, \ldots, j_{m}}^{(m)}$ is the operator acting as $A^{(m)}$ on the variables $x_{j_{1}}, \ldots, x_{j_{m}}$ and as identity on all other variables. Further, we define the corresponding operator $\mathbb{A}_{N}^{(m)}$ on $\mathcal{F}_{\perp}$ as

$$
\mathbb{A}_{N}^{(m)}:=U_{N, \varphi} \mathcal{A}_{N}^{(m)} U_{N, \varphi}^{*} \oplus 0
$$

We construct an asymptotic expansion of $P_{N}^{(n)}$, in the sense that

$$
\operatorname{Tr}_{\mathfrak{H}^{N}} A_{1, \ldots, m}^{(m)} P_{N}^{(n)}=\operatorname{Tr}_{\mathcal{F}_{\perp}} \mathbb{A}_{N}^{(m)} \mathbb{P}_{0}^{(n)}+\lambda_{N}^{\frac{1}{2}} \operatorname{Tr}_{\mathcal{F}_{\perp}} \mathbb{A}_{N}^{(m)} \mathbb{P}_{1}^{(n)}+\lambda_{N} \operatorname{Tr}_{\mathcal{F}_{\perp}} \mathbb{A}_{N}^{(m)} \mathbb{P}_{2}^{(n)}+\cdots
$$

The coefficients $\mathbb{P}_{\ell}^{(n)}$ in the expansion of the projector are defined as follows:

Definition 3.3. Define

$$
\mathbb{P}_{\ell}^{(n)}:= \begin{cases}\mathbb{P}_{0}^{(n)} & \text { if } \ell=0, \\ -\sum_{\nu=1}^{\ell} \sum_{\substack{\boldsymbol{j} \in \mathbb{N}^{v} \\|\boldsymbol{j}|=\ell}} \sum_{\substack{\boldsymbol{k} \in \mathbb{N}_{0}^{v+1} \\|\boldsymbol{k}|=v}} \mathbb{O}_{k_{1}}^{(n)} \mathbb{H}_{j_{1}} \mathbb{O}_{k_{2}}^{(n)} \mathbb{H}_{j_{2}} \cdots \mathbb{O}_{k_{v}}^{(n)} \mathbb{H}_{j_{v}} \mathbb{O}_{k_{v+1}}^{(n)} & \text { if } \ell \geq 1,\end{cases}
$$

with $\mathbb{P}_{0}^{(n)}$ as in Definition 2.4. Here, we have abbreviated

$$
\mathbb{O}_{k}^{(n)}:= \begin{cases}-\mathbb{P}_{0}^{(n)} & k=0 \\ \frac{\mathbb{Q}_{0}^{(n)}}{\left(E_{0}^{(n)}-\mathbb{H}_{0}\right)^{k}} & k>0\end{cases}
$$

and

$$
\begin{aligned}
\mathbb{H}_{1} & :=\mathbb{K}_{3}+\mathbb{K}_{3}^{*}, \\
\mathbb{H}_{2} & :=-\left(\mathcal{N}_{\perp}-1\right) \mathbb{K}_{1}-\left(\mathbb{K}_{2}\left(\mathcal{N}_{\perp}-\frac{1}{2}\right)+\text { h.c. }\right)+\mathbb{K}_{4}, \\
\mathbb{H}_{2 j-1} & :=c_{j-1}\left(\mathbb{K}_{3}\left(\mathcal{N}_{\perp}-1\right)^{j-1}+\text { h.c. }\right), \\
\mathbb{H}_{2 j}: & : \sum_{v=0}^{j} d_{j, v}\left(\mathbb{K}_{2}\left(\mathcal{N}_{\perp}-1\right)^{v}+\text { h.c. }\right)
\end{aligned}
$$


for $j \geq 2$, with $\mathbb{K}_{j}$ as in definition (2.41). The coefficients $c_{j}$ and $d_{j, v}$ are given as

$$
\begin{aligned}
c_{0}^{(\ell)} & :=1, \\
c_{j}^{(\ell)} & :=\frac{\left(\ell-\frac{1}{2}\right)\left(\ell+\frac{1}{2}\right)\left(\ell+\frac{3}{2}\right) \cdots\left(\ell+j-\frac{3}{2}\right)}{j !}, \quad c_{j}:=c_{j}^{(0)} \quad(j \geq 1), \\
d_{j, v} & :=\sum_{\ell=0}^{v} c_{\ell}^{(0)} c_{v-\ell}^{(0)} c_{j-v}^{(\ell)} \quad(j \geq v \geq 0) .
\end{aligned}
$$

Our main result is the following:

Theorem 1. Let Assumptions 1,2 and 3 be satisfied and let $a \in \mathbb{N}_{0}$. Let $m \in \mathbb{N}$ and let $A^{(m)}$ be $a$ self-adjoint operator on $\mathfrak{S}^{m}$ such that

$$
\left\|A^{(m)} \psi\right\|_{\mathfrak{S}^{m}} \leq \mathfrak{C}\left\|\sum_{j=1}^{m}\left(T_{j}+1\right) \psi\right\|_{\mathfrak{S}^{m}} \quad \text { for } \psi \in \mathcal{D}\left(\sum_{j=1}^{m} T_{j}\right) .
$$

Then, for sufficiently large $N$, there exists a constant $\mathfrak{C}(n, m, a)$ such that

$$
\left|\operatorname{Tr}_{\mathfrak{Y}^{N}} \mathcal{A}_{N}^{(m)} P_{N}^{(n)}-\sum_{\ell=0}^{a} \lambda_{N}^{\frac{\ell}{2}} \operatorname{Tr}_{\mathcal{F}_{\perp}} \mathbb{A}_{N}^{(m)} \mathbb{P}_{\ell}^{(n)}\right| \leq \mathfrak{C}(n, m, a) \lambda_{N}^{\frac{a+2}{2}} .
$$

In particular, Theorem 1 proves the validity of Bogoliubov theory up to an error of order $\mathcal{O}\left(N^{-1}\right)-$ that is,

$$
\operatorname{Tr}_{\mathfrak{Y}^{N}} \mathcal{A}_{N}^{(m)} P_{N}^{(n)}=\operatorname{Tr}_{\mathcal{F}_{\perp}} \mathbb{A}_{N}^{(m)} \mathbb{P}_{0}^{(n)}+\mathcal{O}\left(\lambda_{N}\right)
$$

which improves previously known error estimates of order $\mathcal{O}\left(\lambda_{N}^{1 / 2}\right)$.

The coefficients $\operatorname{Tr}_{\mathcal{F}_{\perp}} \mathbb{A}_{N}^{(m)} \mathbb{P}_{\ell}^{(n)}$ in formula (3.12) are not necessarily $N$-independent, because $\mathbb{A}_{N}^{(m)}$ arises from conjugating an operator $\mathcal{A}_{N}^{(m)}$ on the $N$-body Hilbert space with the $N$-dependent unitary map $U_{N, \varphi}$. Unless $A^{(m)}$ is an operator acting only on $\mathfrak{H}_{\perp}^{m}$ (such as, e.g., $A^{(1)}=q$ ), this conjugation yields factors $\sqrt{N-\mathcal{N}_{\perp}}$ comparable to equation (2.40). Hence, to extract the $N$-independent contributions in each order, one needs to expand $\mathbb{A}_{N}^{(m)}$ in $\lambda_{N}^{1 / 2}$ up to the order of the approximation. Equivalently, one derives in this way an expansion of the reduced $m$-particle density matrices of $P_{N}^{(n)}$. For example, the one-particle reduced density matrix

$$
\gamma_{1 ; N}^{(n)}:=\operatorname{Tr}_{\mathfrak{H}^{N-1}} P_{N}^{(n)}
$$

admits the asymptotic expansion

$$
\operatorname{Tr}_{\mathfrak{W}}\left|\gamma_{1 ; N}^{(n)}-\sum_{\ell=0}^{a} \lambda_{N}^{\ell} \widetilde{\gamma}_{1 ; \ell}^{(n)}\right| \leq \mathfrak{C}(n, a) \lambda_{N}^{a+1}
$$

where the coefficients $\widetilde{\gamma}_{1 ; \ell}^{(n)} \in \mathcal{L}(\mathfrak{H})$ are independent of $N$ and can be retrieved as already described. For example, the first correction to the leading order $\widetilde{\gamma}_{1 ; 0}^{(n)}=\delta_{0}^{(n)}|\varphi\rangle\langle\varphi|$ is given by

$$
\begin{aligned}
\widetilde{\gamma}_{1 ; 1}^{(n)}(x ; y) & =\varphi(x) \operatorname{Tr}_{\mathcal{F}_{\perp}} a_{y}^{\dagger} \mathbb{P}_{1}^{(n)}+\varphi(y) \operatorname{Tr}_{\mathcal{F}_{\perp}} a_{x} \mathbb{P}_{1}^{(n)} \\
& +\operatorname{Tr}_{\mathcal{F}_{\perp}} a_{y}^{\dagger} a_{x} \mathbb{P}_{0}^{(n)}-\varphi(x) \varphi(y) \operatorname{Tr}_{\mathcal{F}_{\perp}} \mathbb{P}_{0}^{(n)} \mathcal{N}_{\perp}
\end{aligned}
$$


(see also [11, Theorem 2] for the dynamical counterpart of this statement). For the ground state of a homogeneous Bose gas on the torus, a corresponding result was recently shown in [51], using different methods. Note that in this case, the first line in equation (3.15) vanishes by translation invariance. ${ }^{2}$

Theorem 1 yields an asymptotic expansion of the projector $\mathbb{P}^{(n)}$ onto the subspace $\mathfrak{E}^{(n)}$ of the excitation Fock space, which is defined as

$$
\mathfrak{E}^{(n)}=\bigoplus_{\nu \in \iota^{(n)}} \widetilde{\mathfrak{E}}^{(\nu)}, \quad \widetilde{\mathfrak{E}}^{(v)}=\left\{\phi \oplus 0: \boldsymbol{\phi} \in \mathcal{F}_{\perp}^{\leq N}, \mathbb{H}_{\leq N} \boldsymbol{\phi}=E^{(\nu)} \phi\right\}
$$

(see Definition 3.10). The following statement is proven in Section 5.3.2:

Corollary 3.4. Let $a \in \mathbb{N}_{0}$. Under Assumptions 1,2 and 3 , there exists a constant $\mathfrak{C}(n, a)$ such that

$$
\operatorname{Tr}_{\mathcal{F}_{\perp}}\left|\mathbb{P}^{(n)}-\sum_{\ell=0}^{a} \lambda_{N}^{\frac{\ell}{2}} \mathbb{P}_{\ell}^{(n)}\right| \leq \mathfrak{C}(n, a) \lambda_{N}^{\frac{a+1}{2}}
$$

for sufficiently large $N$.

By means of Bogoliubov transformations, the operators $\mathbb{P}_{\ell}^{(n)}$ can be brought into a more explicit form. For example, the first-order correction for the ground state $(n=0)$ is given by

$$
\mathbb{P}_{1}^{(0)}=\mathbb{U}_{\mathcal{V}_{0}}^{*}\left(\mathbb{U}_{\mathcal{V}_{0}} \mathbb{O}_{1}^{(0)} \mathbb{U}_{\mathcal{V}_{0}}^{*}\right)\left(\mathbb{U}_{\mathcal{V}_{0}} \mathbb{H}_{1} \mathbb{U}_{\mathcal{V}_{0}}^{*}\right)|\Omega\rangle\left\langle\chi_{0}^{(0)}\right|+\text { h.c. }
$$

where $\mathbb{U}_{\mathcal{V}_{0}}$ is the Bogoliubov transformation diagonalising $\mathbb{H}_{0}$ such that $\chi_{0}^{(0)}=\mathbb{U}_{\mathcal{V}_{0}}^{*}|\Omega\rangle$. As the action of $\mathbb{U}_{\mathcal{V}_{0}}$ on creation/annihilation operators is known (see equation (4.15)), it follows that $\mathbb{U}_{\mathcal{V}_{0}} \mathbb{H}_{1} \mathbb{U}_{\mathcal{V}_{0}}^{*}|\Omega\rangle$ is a superposition of one- and three-particle states. Moreover,

$$
\mathbb{U}_{\mathcal{V}_{0}} \mathbb{O}_{1}^{(0)} \mathbb{U}_{\mathcal{V}_{0}}^{*}=\sum_{\ell>0} \sum_{m=1}^{\delta_{0}^{(\ell)}} \frac{1}{E_{0}^{(0)}-E_{0}^{(\ell)}} \mathbb{U}_{\mathcal{V}_{0}}\left|\chi_{0}^{(\ell, m)}\right\rangle\left\langle\chi_{0}^{(\ell, m)}\right| \mathbb{U}_{\mathcal{V}_{0}}^{*},
$$

where $\left\{\chi_{0}^{(\ell, m)}\right\}_{1 \leq m \leq \delta_{0}^{(\ell)}}$ denotes a basis of the eigenspace $\mathfrak{E}_{0}^{(\ell)}$ of $\mathbb{H}_{0}$ and can be written as

$$
\chi_{0}^{(\ell, m)}=\mathbb{U}_{\mathcal{V}_{0}^{*}}^{*} \frac{\left(a^{\dagger}\left(\xi_{0}\right)\right)^{\nu_{0}}}{\sqrt{v_{0} !}} \frac{\left(a^{\dagger}\left(\xi_{1}\right)\right)^{\nu_{1}}}{\sqrt{v_{1} !}} \cdots \frac{\left(a^{\dagger}\left(\xi_{k}\right)\right)^{v_{k}}}{\sqrt{v_{k} !}}|\Omega\rangle
$$

for suitable $\xi_{j} \in \mathfrak{H}_{\perp}, k \in \mathbb{N}_{0}$ and $\left(v_{0}, \ldots, v_{k}\right) \in \mathbb{N}_{0}^{k+1}$ depending on $\ell$ and $m$ (see Lemma 4.7c). Since $\mathbb{U}_{\mathcal{V}_{0}} \mathbb{O}_{1}^{(0)} \mathbb{U}_{\mathcal{V}_{0}}^{*}$ is particle-number preserving, only the basis elements $\chi_{0}^{(\ell, m)}$ with one and three particles contribute to equation (3.17), and applying $\mathbb{U}_{\mathcal{V}_{0}}^{*}$ to the result yields an explicit formula for $\mathbb{P}_{1}^{(0)}$. The general case $(n \geq 0, \ell \geq 1)$ can be treated analogously.

In our second main result, we derive from Theorem 1 an expansion of the low-energy spectrum of $H_{N}$ with $N$-independent coefficients:

Theorem 2. Let $n \in \mathbb{N}_{0}$. Under Assumptions 1,2 and 3 , it holds for any $a \in \mathbb{N}_{0}$ and sufficiently large $N$ that

$$
\left|\frac{1}{\delta_{0}^{(n)}} \sum_{v \in \iota} \delta_{N}^{(v)} \mathscr{E}_{N}^{(v)}-N e_{H}-\sum_{\ell=0}^{a} \lambda_{N}^{\ell} E_{\ell}^{(n)}\right| \leq \mathfrak{C}(n, a) \lambda_{N}^{a+1}
$$

\footnotetext{
${ }^{2}$ In this case, one computes $\tilde{\gamma}_{1 ; 1}^{(0)}=-\sum_{k \neq 0} \gamma_{k}^{2}|\varphi\rangle\left\langle\varphi\left|+\sum_{k \neq 0} \gamma_{k}^{2}\right| \varphi_{k}\right\rangle\left\langle\varphi_{k}\right|$, where $\varphi_{k}=\mathrm{e}^{\mathrm{i} k \cdot x}, \varphi=\varphi_{0}, \gamma_{k}=$ $\alpha_{k}\left(1-\alpha_{k}^{2}\right)^{-1 / 2}$ and $\alpha_{k}=\widehat{v}(k)\left(k^{2}+\widehat{v}(k)+\sqrt{k^{4}+2 k^{2} \widehat{v}(k)}\right)^{-1}$, where $\widehat{v}$ denotes the Fourier transform of $v$.
} 
for some constant $\mathfrak{C}(n, a)$ and for $\iota^{(n)}, \delta_{N}^{(v)}, \mathscr{E}_{N}^{(v)}$ and $\delta_{0}^{(n)}$ as in Definitions 2.4 and 3.1. The coefficients are given as

$$
E_{\ell}^{(n)}:=\frac{1}{\delta_{0}^{(n)}} \sum_{\nu=1}^{2 \ell} \sum_{\substack{\boldsymbol{j} \in \mathbb{N}^{v} \\|\boldsymbol{j}|=2 \ell}} \sum_{\substack{\boldsymbol{m} \in \mathbb{N}_{0}^{v-1} \\|\boldsymbol{m}|=\nu-1}} \frac{1}{\kappa(\boldsymbol{m})} \operatorname{Tr}_{\mathcal{F}_{\perp}} \mathbb{P}_{0}^{(n)} \mathbb{H}_{j_{1}} \mathbb{O}_{m_{1}}^{(n)} \cdots \mathbb{H}_{j_{v-1}} \mathbb{O}_{m_{\nu-1}}^{(n)} \mathbb{H}_{j_{\nu}}
$$

for $\mathbb{O}_{m}^{(n)}$ as in Definition 3.3, and where

$$
\kappa(\boldsymbol{m}):=1+\left|\left\{\mu: m_{\mu}=0\right\}\right| \in\{1, \ldots, v-1\}
$$

is the number of operators $\mathbb{P}_{0}^{(n)}$ within the trace.

All half-integer powers of $\lambda_{N}$ vanish by parity. Equivalently, this can be understood as a consequence of Wick's rule (Lemma 4.6) and the fact that the eigenstates of $\mathbb{H}_{0}$ are given explicitly as Bogoliubov transformations of states with fixed particle number (Lemma $4.7 \mathrm{c}$ ). Moreover, note that the contribution to definition (3.21) from each $v$ decomposes into products of $\kappa(\boldsymbol{m})$ inner products.

Theorem 2 recovers the expressions from perturbation theory as discussed in the introduction. In particular, for any $n \in \mathbb{N}_{0}$ such that $\delta_{0}^{(n)}=1$ (which applies, e.g., to the ground state), $\mathscr{C}_{N}^{(n)}$ is a nondegenerate eigenvalue of $H_{N}$, and the estimate (3.20) reduces to

$$
\mathscr{E}_{N}^{(n)}=N e_{\mathrm{H}}+\sum_{\ell=0}^{a} \lambda_{N}^{\ell} E_{\ell}^{(n)}+\mathcal{O}\left(\lambda_{N}^{a+1}\right) .
$$

In this case, the first two coefficients in the estimate (3.20) simplify to

$$
\begin{aligned}
E_{1}^{(n)}= & \left\langle\chi_{0}^{(n)}, \mathbb{H}_{2} \chi_{0}^{(n)}\right\rangle+\left\langle\chi_{0}^{(n)}, \mathbb{H}_{1} \frac{\mathbb{Q}_{0}^{(n)}}{E_{0}^{(n)}-\mathbb{H}_{0}} \mathbb{H}_{1} \chi_{0}^{(n)}\right\rangle \\
E_{2}^{(n)}= & \sum_{\substack{\nu=1 \\
j}}^{4} \sum_{\substack{|\boldsymbol{j}|=4\\
}}\left\langle\chi_{0}^{(n)}, \mathbb{H}_{j_{1}} \frac{\mathbb{Q}_{0}^{(n)}}{E_{0}^{(n)}-\mathbb{H}_{0}} \mathbb{H}_{j_{2}} \cdots \frac{\mathbb{Q}_{0}^{(n)}}{E_{0}^{(n)}-\mathbb{H}_{0}} \mathbb{H}_{j_{v}} \chi_{0}^{(n)}\right\rangle \\
& -E_{1}^{(n)}\left\langle\chi_{0}^{(n)}, \mathbb{H}_{1} \frac{\mathbb{Q}_{0}^{(n)}}{\left(E_{0}^{(n)}-\mathbb{H}_{0}\right)^{2}} \mathbb{H}_{1} \chi_{0}^{(n)}\right\rangle .
\end{aligned}
$$

Remark 3.5. Theorem 1 holds for any fixed $n \in \mathbb{N}_{0}, a \in \mathbb{N}_{0}$ and $m \in \mathbb{N}$ for sufficiently large $N$, with an error $\mathfrak{C}(n, m, a)$ that is not uniform in $n, m$ or $a$. In particular, $\mathfrak{C}(n, m, a)$ depends on $\left|E_{0}^{(n)}\right|$, hence the statement is nontrivial only for eigenvalues of $H_{N}$ of order 1 above the ground-state energy.

Moreover, $\mathfrak{C}(n, m, a)$ grows rapidly in the order $a$ of the approximation. In the special case where $v \in L^{\infty}\left(\mathbb{R}^{d}\right)$, our estimates imply that

$$
\mathfrak{C}(n, m, a) \leq(\mathfrak{C}(n, m)(a+1))^{(a+6)^{2}},
$$

and the bound is certainly worse in the general case (see Remark 3.15). We do not expect this estimate to be optimal, especially as Borel summability was proven for a comparable perturbative expansion of the mean-field dynamics on Fock space for bounded interactions [25]. Also in that setting, the available estimates for unbounded potentials are worse and, in particular, insufficient to conclude Borel summability [24]. 
Remark 3.6. As explained in Section 2.1, Assumptions 1, 2 and 3 are satisfied, for example, by bounded positive definite potentials and by the repulsive Coulomb potential in $d=3$. These assumptions ensure that Bogoliubov theory is valid for our model - that is, that all assumptions in [36] are satisfied. In that work, it is shown that $\mathbb{H}_{0}$ approximates $\mathbb{H}$ to leading order for any self-adjoint $T$ that is bounded from below, and for interaction potentials

$$
-c_{1}\left(T_{1}+T_{2}+c_{2}\right) \leq v\left(x_{1}-x_{2}\right) \leq c_{3}\left(T_{1}+T_{2}+1\right), \quad 0<c_{1}<1, \quad c_{2}, c_{3}>0
$$

[36, (A1)], such that there exists a unique nondegenerate minimiser for the Hartree functional and such that the operators $K_{1}$ and $K_{2}$ from definitions (2.42) $\left(K_{2}\right.$ as operator $\left.\mathfrak{H}^{*} \rightarrow \mathfrak{G}\right)$ are Hilbert-Schmidt [36, (A2)]. Moreover, it is required that

$$
H_{N}-N e_{\mathrm{H}} \geq c \sum_{j=1}^{N} h_{j}+\mathcal{O}(N)
$$

for some $0<c<1$ [36, (A3s)]. Our analysis, which can be understood as a perturbative expansion of $\mathbb{H}$ around the leading order $\mathbb{H}_{0}$, relies on the result proven in [36]: we need $E^{(v)} \approx E_{0}^{(n)}$ (for sufficiently large $N$ ) to find a suitable contour $\gamma^{(n)}$ enclosing $E_{0}^{(n)}$ as well as all $E^{(v)}$ with $v \in \iota^{(n)}$, and we require that $\chi^{(n)} \rightarrow \chi_{0}^{(n)}$ strongly in the norm induced by the quadratic form of $\mathbb{H}_{0}$ to conclude that $\left\langle\chi^{(n)}, \mathcal{N}_{\perp} \chi^{(n)}\right\rangle$ is bounded uniformly in $N$ (see Lemma 4.8).

In contrast to the generic setting from [36], we choose $T=-\Delta+V^{\text {ext }}$ and consider a positive definite interaction $v$ satisfying the stronger bound (2.3), which implies [34, (A1-A2)] (see Lemma 2.2). In particular, the bound (2.3) is crucial to bounding $\mathbb{K}_{3}$ by powers of $\mathcal{N}_{\perp}$, and $\mathbb{K}_{4}$ in terms of $\mathrm{d} \Gamma_{\perp}(h)^{1 / 2}$ and powers of $\mathcal{N}_{\perp}$. Moreover, Assumption 3 is stronger than [36, (A3s)] since we require an error of at most $\mathcal{O}\left(N^{1 / 3}\right)$ to control arbitrary moments of $\mathcal{N}_{\perp}$ with respect to $\chi^{(n)}$, as explained later.

Our analysis generalises to certain interactions $v$ which are not of positive type, and to a class of confining potentials $V^{\text {ext }}$ that do not diverge at infinity. More precisely, we can cover all potentials $v$ and $V^{\text {ext }}$ such that all assumptions in [36] and Assumption 3 are satisfied. For example, it is shown in [36, Section 3.2] that a trapped two-dimensional gas with repulsive Coulomb interactions and $V^{\text {ext }}$ diverging sufficiently fast at infinity,

$$
H_{N}=\sum_{j=1}^{N}\left(-\Delta_{j}+V^{\mathrm{ext}}\left(x_{j}\right)\right)-\lambda_{N} \sum_{i<j} \ln \left|x_{i}-x_{j}\right|, \quad d=2,
$$

satisfies [36, (A1-A3s)] as well as Assumption 3 [36, Lemma 3.7], although $v(x)=-\ln |x|$ is not of positive type. Moreover, it is explained in [36, Section 3.2] that bosonic atoms below a critical binding number $t_{c}$, which are described by the rescaled Hamiltonian

$$
H_{t, N}=\sum_{j=1}^{N}\left(-\Delta_{j}-\frac{1}{t\left|x_{j}\right|}\right)+\lambda_{N} \sum_{i<j} \frac{1}{\left|x_{i}-x_{j}\right|}, \quad t<t_{c} \in(1,2), \quad d=3,
$$

meet all criteria, including our Assumption 3. Other viable choices for $T$ are the Laplace operator on a bounded subset of $\mathbb{R}^{d}$ with Dirichlet, Neumann or periodic boundary conditions, or relativistic kinetic terms.

Finally, we construct an asymptotic expansion of the $N$-body eigenstates $\Psi_{N}^{(n)}$ of $H_{N}$ that correspond to nondegenerate eigenvalues of $\mathbb{H}_{0}$ :

Theorem 3. Let $a \in \mathbb{N}_{0}$ and let Assumptions 1,2 and 3 be satisfied. Assume that $n \in \mathbb{N}_{0}$ such that $\delta_{0}^{(n)}=1$ and let $\Psi_{N}^{(n)} \in \mathfrak{E}_{N}^{(n)}$. Then for a suitable choice of the phase of $\chi_{0}^{(n)}$, there exists a constant 
$\mathfrak{C}(n, a)$ such that

$$
\left\|\Psi_{N}^{(n)}-\sum_{\ell=0}^{a} \lambda_{N}^{\frac{\ell}{2}} \sum_{k=0}^{N} \varphi^{\otimes(N-k)} \otimes_{S}\left(\chi_{\ell}^{(n)}\right)^{(k)}\right\|_{\mathfrak{H}^{N}} \leq \mathfrak{C}(n, a) \lambda_{N^{\frac{a+1}{2}}}
$$

for sufficiently large $N$, where

$$
\begin{aligned}
\chi_{\ell}^{(n)} & :=\sum_{j=0}^{\ell} \alpha_{j} \widetilde{\chi}_{\ell-j}^{(n)} \quad(\ell \geq 1), \\
\widetilde{\chi}_{\ell}^{(n)} & :=\sum_{v=1}^{\ell} \sum_{\substack{j \in \mathbb{N}^{v} \\
|j|=\ell}} \mathbb{P}_{j_{1}}^{(n)} \cdots \mathbb{P}_{j_{v}}^{(n)} \chi_{0}^{(n)} \quad(\ell \geq 1),
\end{aligned}
$$

and with

$$
\alpha_{0}:=1, \quad \alpha_{2 n-1}:=0, \quad \alpha_{2 n}:=-\frac{1}{2} \sum_{\substack{\boldsymbol{j} \in \mathbb{N}_{0}^{4} \\ j_{1}, j_{2}<2 n \\ j|\boldsymbol{j}|=2 n}} \alpha_{j_{1}} \alpha_{j_{2}}\left\langle\widetilde{\boldsymbol{\chi}}_{j_{3}}^{(n)}, \widetilde{\boldsymbol{\chi}}_{j_{4}}^{(n)}\right\rangle
$$

for $n \geq 1$.

Theorem 3 is an immediate consequence of a much more general statement: if a rank 1 projector admits an asymptotic expansion in a small parameter $\varepsilon$, this implies an asymptotic expansion of the corresponding wave function. Since we could not find any proof of this seemingly obvious assertion, we prove it for a generic perturbative setting in Appendix B. By parity, the parameters $\alpha_{\ell}$ vanish for $\ell$ odd, which can be seen analogously to the vanishing of the half-integer powers of $\lambda_{N}$ in Theorem 2 . Note that the estimate (3.25) also holds with $\chi_{\ell}^{(n)}$ replaced by $\widetilde{\chi}_{\ell}^{(n)}$ times an overall factor $\alpha^{(a)}=\sum_{\ell=0}^{a} \lambda_{N}^{\ell / 2} \alpha_{\ell}$.

Remark 3.7. Recall that each Bogoliubov eigenstate $\chi_{0}^{(n)}$ can be expressed as a Bogoliubov transformation $\mathbb{U}_{\mathcal{V}_{0}}^{*}$ of a wave function with fixed particle number $m_{n} \in \mathbb{N}_{0}$ (see Lemma $4.7 \mathrm{c}$ ). Consequently, $\chi_{\ell}^{(n)}$ can be written as $\mathbb{U}_{\mathcal{V}_{0}}^{*}$ acting on a superposition of wave functions with $\mu \leq m_{n}+3 \ell$ particles, with $\mu+\ell+m_{n}$ even. To see this, note that $\mathbb{U}_{\mathcal{V}_{0}} \mathbb{O}_{k}^{(n)} \mathbb{U}_{\mathcal{V}_{0}}^{*}$ is particle-number preserving, and $\mathbb{U}_{\mathcal{V}_{0}} \mathbb{H}_{j} \mathbb{U}_{\mathcal{V}_{0}}^{*}$ has even (resp., odd) parity for $j$ even (resp., odd) and contains at most $j+2$ creation operators. Hence, the maximum number of creation operators in definition (3.26b) is contributed by $v=1$, namely by the term containing exclusively operators $\mathbb{H}_{1}$ and exactly one operator $\mathbb{P}_{0}$ (i.e., $v=\ell, \boldsymbol{j}=(1,1, \ldots, 1)$ and $\boldsymbol{k}=(1,1, \ldots, 1,0)$ in definition (3.7)). Such initial data are used for a perturbative expansion of the dynamics of the Bose gas in the mean-field limit in [11].

Remark 3.8. For any given $\ell \in \mathbb{N}$, expression (3.26b) can be simplified further, since many terms vanish by parity and most of the remaining terms can be grouped into summands which differ only by a prefactor (compare equations (3.28)); for example,

$$
\widetilde{\chi}_{2}^{(n)}=\left(\mathbb{P}_{2}^{(n)}+\mathbb{P}_{1}^{(n)} \mathbb{P}_{1}^{(n)}\right) \chi_{0}^{(n)}=\left(\mathbb{O}_{2}^{(n)} \mathbb{H}_{2}+\mathbb{O}_{1}^{(n)} \mathbb{H}_{1} \mathbb{O}_{1}^{(n)} \mathbb{H}_{1}\right) \chi_{0}^{(n)}
$$

The approximating wave functions in Theorem 3 are constructed via the eigenvalue equation $\sum_{\ell=0}^{\infty} \lambda_{N}^{\ell / 2} \mathbb{P}_{\ell}^{(n)} \chi^{(n)}=\chi^{(n)}$ (see Appendix B). Alternatively, one can (formally) derive simpler formulas for both $\widetilde{\chi}_{\ell}^{(n)}$ and the coefficients $E_{\ell}^{(n)}$ from Theorem 2 by an analogous construction for the eigenvalue 
equation $\mathbb{H} \mathcal{\chi}^{(n)}=E^{(n)} \chi^{(n)}$. A formal computation yields

$$
\begin{aligned}
\tilde{\chi}_{\ell}^{(n)} & =\sum_{v=1}^{\ell} \sum_{\substack{\boldsymbol{j} \in \mathbb{N}^{v} \\
|\boldsymbol{j}|=\ell}} \mathbb{O}_{1}^{(n)} \mathbb{H}_{j_{1}}^{\prime} \cdots \mathbb{O}_{1}^{(n)} \mathbb{H}_{j_{v-1}}^{\prime} \mathbb{O}_{1}^{(n)} \mathbb{H}_{j_{v}} \chi_{0}^{(n)}, \\
E_{\ell}^{(n)} & =\sum_{\nu=1}^{2 \ell} \sum_{\substack{\boldsymbol{j} \in \mathbb{N}^{v} \\
|\boldsymbol{j}|=2 \ell}}\left\langle\chi_{0}^{(n)}, \mathbb{H}_{j_{1}} \mathbb{O}_{1}^{(n)} \mathbb{H}_{j_{2}}^{\prime} \cdots \mathbb{O}_{1}^{(n)} \mathbb{H}_{j_{v-1}^{\prime}} \mathbb{O}_{1}^{(n)} \mathbb{H}_{j_{v}} \chi_{0}^{(n)}\right\rangle,
\end{aligned}
$$

where $\mathbb{H}_{j}^{\prime}=\mathbb{H}_{j}$ for $j$ odd and $\mathbb{H}_{j}^{\prime}=\mathbb{H}_{j}-E_{j / 2}^{(n)}$ for $j$ even. Here, $\widetilde{\chi}_{\ell}^{(n)}$ is given in terms of the coefficients $E_{\ell}^{(n)}$, which are determined iteratively. For the first few orders, one easily verifies that the equations (3.28) coincide with the expressions from Theorems 3 and 2 for $\delta_{0}^{(n)}=1$.

\subsection{Strategy of proof}

In the remainder of this section, we explain the proof of Theorems 1 and 2. We begin with extending $\mathbb{H}_{\leq N}$ to the full excitation Fock space $\mathcal{F}_{\perp}$ in the following way:

Definition 3.9. We extend $\mathbb{H}_{\leq N}$ (see Definition 2.3) from $\mathcal{F}_{\perp}^{\leq N}$ to the full Fock space $\mathcal{F}_{\perp}$ as

$$
\mathbb{H}:=\mathbb{H}_{\leq N} \oplus E^{(-1)},
$$

where

$$
E^{(-1)}:=E^{(0)}-\left(E^{(1)}-E^{(0)}\right)
$$

with $E^{(n)}$ the eigenvalues of $\mathbb{H}_{\leq N}$ (see again Definition 2.3). Consequently, the low-energy spectrum of $\mathbb{H}$ consists of the eigenvalues

$$
E^{(-1)}<E^{(0)}<E^{(1)}<\cdots<E^{(n)}<\cdots .
$$

Note that we could have extended $\mathbb{H}_{\leq N}$ to $\mathcal{F}_{\perp}$ in many ways. To motivate the choice of definition (3.29), recall that our aim is to expand the spectral projectors of $\mathbb{H}$ around the corresponding spectral projectors of $\mathbb{H}_{0}$, which we do by expressing them as contour integrals over the resolvent of $\mathbb{H}$ and subsequently expanding $(z-\mathbb{H})^{-1}$ around $\left(z-\mathbb{H}_{0}\right)^{-1}$. Let us first consider the case where the eigenvalues $E^{(n)}$ and $E_{0}^{(n)}$ of $\mathbb{H}$ and $\mathbb{H}_{0}$, respectively, are nondegenerate. In view of equation (1.15), we require an $\mathcal{O}(1)$ contour $\gamma^{(n)}$ that encloses both $E^{(n)}$ and $E_{0}^{(n)}$ and leaves the remaining spectrum of $\mathbb{H}$ outside. The choice $\mathbb{H}=\mathbb{H}_{\leq N} \oplus c$, for $c$ a finite distance away from any point in the spectrum of $\mathbb{H}_{\leq N}$, ensures that $\mathbb{H}$ has precisely one (infinitely degenerate) additional eigenvalue $c$ compared to $\mathbb{H}_{\leq N}$. Since the spectrum of $\mathbb{H}_{\leq N}$ is bounded from below by $E^{(0)}$, we place $c$ at a finite distance below $E^{(0)}$, for simplicity such that the spectral gaps below and above $E^{(0)}$ have the same size.

If $E_{0}^{(n)}$ is degenerate, the expansion must be done carefully, because we cannot exclude the possibility that nondegenerate eigenvalues of $\mathbb{H}$ become degenerate in the limit $N \rightarrow \infty$. By [36], every low-energy eigenvalue of $\mathbb{H}$ converges to an eigenvalue of $\mathbb{H}_{0}$ (see Lemma 4.8a), but the situation may occur that an eigenvalue $E_{0}^{(n)}$ of $\mathbb{H}_{0}$ is, for instance, twice degenerate, and there exist (for any finite $N$ ) two eigenvalues $E^{\left(n_{1}\right)} \neq E^{\left(n_{2}\right)}$ of $\mathbb{H}$ such that

$$
\lim _{N \rightarrow \infty} E^{\left(n_{1}\right)}=E_{0}^{(n)}=\lim _{N \rightarrow \infty} E^{\left(n_{2}\right)}
$$

In this case, it makes sense to expand the sum of the corresponding projectors around $\mathbb{P}_{0}^{(n)}$, which becomes apparent when recalling equation (1.15): since each closed curve of order 1 around $E_{0}^{(n)}$ must 
enclose both poles $E^{\left(n_{1}\right)}$ and $E^{\left(n_{2}\right)}$ of $(z-\mathbb{H})^{-1}$, the contour integral gives precisely the sum of the two spectral projections. This motivates the following definition:

Definition 3.10. For any $n \in \mathbb{N}_{0}$, we define the path

$$
\gamma^{(n)}:=\left\{E_{0}^{(n)}+\mathfrak{g}^{(n)} \mathrm{e}^{\mathrm{i} t}: t \in[0,2 \pi)\right\} \subset \mathbb{C},
$$

where

$$
\mathfrak{g}^{(n)}:=\frac{1}{2} \min \left\{g_{0}^{(n-1)}, g_{0}^{(n)}\right\}
$$

for $g_{0}^{(n)}$ as in definition (2.47). For $n \in \mathbb{N}_{0}$, define

$$
\mathbb{P}^{(n)}:=\frac{1}{2 \pi \mathrm{i}} \oint_{\gamma^{(n)}} \frac{1}{z-\mathbb{H}} \mathrm{d} z, \quad \mathbb{Q}^{(n)}:=\mathbb{1}_{\mathcal{F}_{\perp}}-\mathbb{P}^{(n)}
$$

and

$$
\mathfrak{E}^{(n)}:=\mathbb{P}^{(n)} \mathcal{F}_{\perp}=\bigoplus_{\nu \in \iota^{(n)}} \widetilde{\mathfrak{F}}^{(v)} \subset \mathcal{F}_{\perp}^{\leq N} \oplus 0
$$

with $\iota^{(n)}$ as in definition (3.1) and where $\widetilde{\mathfrak{E}}^{(v)}$ denotes the eigenspace of $\mathbb{H}$ at $E^{(v)}$,

$$
\widetilde{\mathfrak{E}}^{(v)}:=\left\{\phi \oplus 0: \boldsymbol{\phi} \in \mathcal{F}_{\perp}^{\leq N}, \mathbb{H}_{\leq N} \boldsymbol{\phi}=E^{(v)} \boldsymbol{\phi}\right\},
$$

with dimension $\delta_{N}^{(v)}$ as in definition (3.3). We denote normalised elements of $\mathfrak{E}^{(n)}$ as

$$
\chi^{(n)}:=\chi_{\leq N}^{(n)} \oplus 0 .
$$

For $n=-1$, we define $\mathbb{P}^{(-1)}$ as the projector onto the eigenspace of $\mathbb{H}$ associated with $E^{(-1)}$,

$$
\mathfrak{E}^{(-1)}:=\left\{0 \oplus \boldsymbol{\phi}: \boldsymbol{\phi} \in \mathcal{F}_{\perp}^{>N}\right\}, \quad \mathbb{P}^{(-1)}:=\mathbb{1}_{\mathfrak{E}^{(-1)}} .
$$

Next, we expand $\mathbb{H}$ in powers of $\lambda_{N}^{1 / 2}$. The $N$-dependence in $\mathbb{H}$ has two sources: first, $\mathbb{H}$ is defined as the direct sum of $\mathbb{H}_{\leq N}$ on $\mathcal{F}_{\perp}^{\leq N}$ and a conveniently chosen constant on $\mathcal{F}_{\perp}^{>N}$; second, the operators in $\mathbb{H}_{\leq N}$ come with $N$-dependent prefactors. To deal with the first point, we write $\mathbb{H}$ on $\mathcal{F}_{\perp}$ as

$$
\mathbb{H}=\mathbb{H}^{<}+\mathbb{H}^{>},
$$

with

$$
\begin{aligned}
& \mathbb{H}^{<}:=\mathbb{K}_{0}+\left(1-\frac{\mathcal{N}_{\perp}-1}{N-1}\right) \mathbb{K}_{1}+\left(\mathbb{K}_{2} \frac{\sqrt{\left[\left(N-\mathcal{N}_{\perp}\right)\left(N-\mathcal{N}_{\perp}-1\right)\right]_{+}}}{N-1}+\text { h.c. }\right) \\
&+\left(\mathbb{K}_{3} \frac{\sqrt{\left[N-\mathcal{N}_{\perp}\right]_{+}}}{N-1}+\text { h.c. }\right)+\frac{1}{N-1} \mathbb{K}_{4}, \\
& \mathbb{H}^{>}:=0 \oplus\left(E^{(-1)}-\mathbb{K}_{0}-\left(1-\frac{\mathcal{N}_{\perp}-1}{N-1}\right) \mathbb{K}_{1}-\frac{1}{N-1} \mathbb{K}_{4}\right),
\end{aligned}
$$

where $[\cdot]_{+}$denotes the positive part. Note that $\mathbb{K}_{0}, \mathbb{K}_{1}$ and $\mathbb{K}_{4}$ conserve the particle number, so the restriction to $\mathcal{F}_{\perp}^{>N}$ in definition (3.40b) makes sense. The first term $\mathbb{H}^{<}$is defined on the full space $\mathcal{F}_{\perp}$. To obtain $\mathbb{H}^{<}$, we add to $\mathbb{H}$ the missing contributions to $\mathbb{K}_{0}, \mathbb{K}_{1}$ and $\mathbb{K}_{4}$ on the sectors $\mathcal{F}_{\perp}^{>}$, and subtract them again in $\mathbb{H}^{>}$. Finally, we expand the square roots from $\mathbb{H}^{<}$in a Taylor series (see [11, Appendix C] for a proof). 
Lemma 3.11. Let $a \in \mathbb{N}_{0}$ and $c_{\ell}^{(j)}$ and $d_{\ell, j}$ as in definition (3.10).

(a) Define the operator $\widetilde{\mathbb{R}}_{a}^{(3)}$ on $\mathcal{F}_{\perp}$ via the identity

$$
\frac{\sqrt{\left[N-\mathcal{N}_{\perp}\right]_{+}}}{N-1}=\sum_{\ell=0}^{a} c_{\ell} \lambda_{N}^{\ell+\frac{1}{2}}\left(\mathcal{N}_{\perp}-1\right)^{\ell}+\lambda_{N}^{a+\frac{3}{2}} \widetilde{\mathbb{R}}_{a}^{(3)}
$$

Then $\left[\widetilde{\mathbb{R}}_{a}^{(3)}, \mathcal{N}_{\perp}\right]=0$ and

$$
\left\|\widetilde{\mathbb{R}}_{a}^{(3)} \phi\right\|_{\mathcal{F}_{\perp}} \leq 2^{a+1}\left\|\left(\mathcal{N}_{\perp}+1\right)^{a+1} \phi\right\|_{\mathcal{F}_{\perp}}
$$

for $\phi \in \mathcal{F}_{\perp}$.

(b) Define the operator $\widetilde{\mathbb{R}}_{a}^{(2)}$ on $\mathcal{F}_{\perp}$ through

$$
\frac{\sqrt{\left[\left(N-\mathcal{N}_{\perp}\right)\left(N-\mathcal{N}_{\perp}-1\right)\right]_{+}}}{N-1}=\sum_{\ell=0}^{a} \lambda_{N}^{\ell} \sum_{j=0}^{\ell} d_{\ell, j}\left(\mathcal{N}_{\perp}-1\right)^{j}+\lambda_{N}^{a+1} \widetilde{\mathbb{R}}_{a}^{(2)}
$$

Then $\left[\widetilde{\mathbb{R}}_{a}^{(2)}, \mathcal{N}_{\perp}\right]=0$ and

$$
\left\|\widetilde{\mathbb{R}}_{a}^{(2)} \phi\right\|_{\mathcal{F}_{\perp}} \leq(a+1)^{2} 4^{a+1}\left\|\left(\mathcal{N}_{\perp}+1\right)^{a+1} \phi\right\|_{\mathcal{F}_{\perp}}
$$

for $\phi \in \mathcal{F}_{\perp}$.

With this, we can expand $\mathbb{H}^{<}$in powers of $\lambda_{N}^{1 / 2}$ :

Proposition 3.12. Let $a \in \mathbb{N}_{0}$. In the sense of operators on $\mathcal{F}_{\perp}$, it holds that

$$
\mathbb{H}^{<}=\sum_{j=0}^{a} \lambda_{N}^{\frac{j}{2}} \mathbb{H}_{j}+\lambda_{N}^{\frac{a+1}{2}} \mathbb{R}_{a}
$$

with $\mathbb{H}_{j}$ as in Definitions 2.4 and 3.3. The remainders are given as

$$
\begin{aligned}
& \mathbb{R}_{0}:=\mathbb{R}_{0}^{(1)}+\lambda_{N}^{\frac{1}{2}} \mathbb{K}_{4}, \\
& \mathbb{R}_{1}:=\mathbb{R}_{1}^{(1)}+\mathbb{K}_{4}
\end{aligned}
$$

and

$$
\begin{aligned}
\mathbb{R}_{0}^{(1)} & :=\left(\mathbb{K}_{3} \sqrt{\frac{\left[N-\mathcal{N}_{\perp}\right]_{+}}{N-1}}+\text { h.c. }\right)+\lambda_{N}^{\frac{1}{2}}\left(\left(\mathbb{K}_{2} \widetilde{\mathbb{R}}_{0}^{(2)}+\text { h.c. }\right)-\left(\mathcal{N}_{\perp}-1\right) \mathbb{K}_{1}\right), \\
\mathbb{R}_{1}^{(1)} & :=-\left(\mathcal{N}_{\perp}-1\right) \mathbb{K}_{1}+\left(\mathbb{K}_{2} \widetilde{\mathbb{R}}_{0}^{(2)}+\text { h.c. }\right)+\lambda_{N}^{\frac{1}{2}}\left(\mathbb{K}_{3} \widetilde{\mathbb{R}}_{0}^{(3)}+\text { h.c. }\right), \\
\mathbb{R}_{2 j} & :=\mathbb{K}_{3} \widetilde{\mathbb{R}}_{j-1}^{(3)}+\lambda_{N}^{\frac{1}{2}} \mathbb{K}_{2} \widetilde{\mathbb{R}}_{j}^{(2)}+\text { h.c. } \\
\mathbb{R}_{2 j+1} & :=\mathbb{K}_{2} \widetilde{\mathbb{R}}_{j}^{(2)}+\lambda_{N}^{\frac{1}{2}} \mathbb{K}_{3} \widetilde{\mathbb{R}}_{j}^{(3)}+\text { h.c. }
\end{aligned}
$$

for $j \geq 1$, with $\widetilde{\mathbb{R}}_{j}^{(2)}$ and $\widetilde{\mathbb{R}}_{j}^{(3)}$ from Lemma 3.11. 
The next step is to expand $\mathbb{P}^{(n)}$ around $\mathbb{P}_{0}^{(n)}$, using the fact that

$$
\mathbb{P}_{0}^{(n)}=\frac{1}{2 \pi \mathrm{i}} \oint_{\gamma^{(n)}} \frac{1}{z-\mathbb{H}_{0}} \mathrm{~d} z
$$

because $\gamma^{(n)}$ from definition (3.32) encloses $E_{0}^{(n)}$. In view of the definition (3.34) of $\mathbb{P}^{(n)}$, we first expand $(z-\mathbb{H})^{-1}$ around $\left(z-\mathbb{H}_{0}\right)^{-1}$ and integrate the resulting expressions along $\gamma^{(n)}$.

Lemma 3.13. Let $a \in \mathbb{N}_{0}$ and $z \in \varrho(\mathbb{H}) \cap \varrho\left(\mathbb{H}_{0}\right)$, where $\varrho$ denotes the resolvent set. Then

$$
\frac{1}{z-\mathbb{H}}=\frac{1}{z-\mathbb{H}_{0}} \sum_{\ell=0}^{a} \lambda_{N}^{\frac{\ell}{2}} \mathbb{T}_{\ell}(z)+\lambda_{N}^{\frac{a+1}{2}} \frac{1}{z-\mathbb{H}^{<}} \mathbb{S}_{a}(z)+\frac{1}{z-\mathbb{H}^{<}} \mathbb{H}^{>} \frac{1}{z-\mathbb{H}},
$$

where

$$
\begin{aligned}
& \mathbb{T}_{\ell}(z)=\sum_{v=1}^{\ell} \sum_{\substack{\boldsymbol{j} \in \mathbb{N}^{v} \\
|\boldsymbol{j}|=\ell}} \mathbb{H}_{j_{1}} \frac{1}{z-\mathbb{H}_{0}} \mathbb{H}_{j_{2}} \frac{1}{z-\mathbb{H}_{0}} \cdots \mathbb{H}_{j_{v}} \frac{1}{z-\mathbb{H}_{0}}, \\
& \mathbb{S}_{a}(z)=\sum_{\nu=0}^{a} \mathbb{R}_{v} \frac{1}{z-\mathbb{H}_{0}} \mathbb{T}_{a-v}(z) .
\end{aligned}
$$

Here, the notation is understood such that $\mathbb{T}_{0}(z)=\mathbb{1}$.

The proof of Lemma 3.13 is postponed to Section 5.1.1. Essentially, one uses the identities

$$
\mathbb{H}^{<}=\mathbb{H}_{0}+\lambda_{N}^{\frac{1}{2}} \mathbb{R}_{0}, \quad \mathbb{R}_{0}=\mathbb{H}_{1}+\lambda_{N}^{\frac{1}{2}} \mathbb{R}_{1},
$$

which follow from Proposition 3.12, to conclude that

$$
\begin{aligned}
\frac{1}{z-\mathbb{H}^{<}} & =\frac{1}{z-\mathbb{H}_{0}}+\lambda_{N}^{\frac{1}{2}} \frac{1}{z-\mathbb{H}^{<}} \mathbb{R}_{0} \frac{1}{z-\mathbb{H}_{0}} \\
& =\frac{1}{z-\mathbb{H}_{0}}+\lambda_{N}^{\frac{1}{2}} \frac{1}{z-\mathbb{H}_{0}} \mathbb{H}_{1} \frac{1}{z-\mathbb{H}_{0}}+\mathcal{O}\left(\lambda_{N}\right),
\end{aligned}
$$

and iterating this procedure up to order $\mathcal{O}\left(\lambda_{N}^{(a+1) / 2}\right)$ concludes the proof.

The next step is to integrate equation (3.48) along the contour $\gamma^{(n)}$ as in equation (3.47). The first term in equation (3.48) gives an integral over products of alternately $\left(z-\mathbb{H}_{0}\right)^{-1}$ and $\mathbb{H}_{j}$. After decomposing $1=\mathbb{P}_{0}^{(n)}+\mathbb{Q}_{0}^{(n)}$ in each resolvent, we note that the term with exclusively $\mathbb{Q}_{0}^{(n)}$ vanishes because the integrand is, by construction, holomorphic in the interior of $\gamma^{(n)}$. The remaining terms, all of which contain at least one projection $\mathbb{P}_{0}^{(n)}$, can be simplified using the residue theorem. Note that $\mathbb{P}_{0}^{(n)} /\left(z-\mathbb{H}_{0}\right)=\mathbb{P}_{0}^{(n)} /\left(z-E_{0}^{(n)}\right)$, hence the number of operators $\mathbb{P}_{0}^{(n)}$ determines the order of the pole at $z=E_{0}^{(n)}$.

The second term in equation (3.48) is of the same structure as the first one but starts with $\left(z-\mathbb{H}^{<}\right)^{-1}$ instead of $\left(z-\mathbb{H}_{0}\right)^{-1}$. For later convenience, we decompose the first identity as $1=\mathbb{P}^{(n)}+\mathbb{Q}^{(n)}$. Moreover, in case of $\mathbb{Q}^{(n)}$, we resolve all remaining identities as $1=\mathbb{P}_{0}^{(n)}+\mathbb{Q}_{0}^{(n)}$ and note that the contribution with $\mathbb{Q}^{(n)}$ and exclusively $\mathbb{Q}_{0}^{(n)}$ vanishes, as the integrand is holomorphic.

Finally, in the last term of equation (3.48) we decompose both identities as $1=\mathbb{P}^{(n)}+\mathbb{Q}^{(n)}$ and observe that $\mathbb{P}^{(n)} \mathbb{H}^{>}=0$ because $\mathbb{P}^{(n)}$ projects onto a subset of $\mathcal{F}_{\perp}^{\leq N}$, where $\mathbb{H}^{>}$equals zero. This leaves 
only the term with twice $\mathbb{Q}^{(n)}$, which vanishes upon integration. In summary, we obtain the following formula for $\mathbb{P}^{(n)}$ :

Proposition 3.14. Let $a \in \mathbb{N}_{0}, n \in \mathbb{N}_{0}$ and $\gamma^{(n)}$ as in definition (3.32). Then

$$
\mathbb{P}^{(n)}=\sum_{\ell=0}^{a} \lambda_{N}^{\frac{\ell}{2}} \mathbb{P}_{\ell}^{(n)}+\lambda_{N}^{\frac{a+1}{2}}\left(\mathbb{B}_{P}^{(n)}(a)+\mathbb{B}_{Q}^{(n)}(a)\right)
$$

for $\mathbb{P}_{\ell}^{(n)}$ as in Definition 3.3 and where

$$
\mathbb{B}_{P}^{(n)}(a)=\sum_{\nu=0}^{a} \sum_{m=1}^{a-v} \sum_{\substack{\boldsymbol{j} \in \mathbb{N}^{m} \\|\boldsymbol{j}|=a-v}} \frac{1}{2 \pi \mathrm{i}} \oint_{\gamma^{(n)}} \frac{\mathbb{P}^{(n)}}{z-\mathbb{H}^{<}} \mathbb{R}_{v} \frac{1}{z-\mathbb{H}_{0}} \mathbb{H}_{j_{1}} \frac{1}{z-\mathbb{H}_{0}} \cdots \mathbb{H}_{j_{m}} \frac{1}{z-\mathbb{H}_{0}} \mathrm{~d} z
$$

and

$$
\begin{aligned}
\mathbb{B}_{Q}^{(n)}(a)= & \sum_{\nu=0}^{a} \sum_{m=1}^{a-v} \sum_{\substack{\boldsymbol{j} \in \mathbb{N}^{m} \\
|\boldsymbol{j}|=a-v}} \sum_{\ell=0}^{m} \sum_{\substack{\boldsymbol{k} \in\{0,1\}^{m+1} \\
|\boldsymbol{k}|=\ell}} \frac{1}{2 \pi \mathrm{i}} \oint_{\gamma^{(n)}} \frac{\mathbb{Q}^{(n)}}{z-\mathbb{H}^{<}} \mathbb{R}_{\nu} \\
& \times \frac{\mathbb{I}_{k_{1}}^{(n)}}{z-\mathbb{H}_{0}} \mathbb{H}_{j_{1}} \frac{\mathbb{I}_{k_{2}}^{(n)}}{z-\mathbb{H}_{0}} \cdots \mathbb{H}_{j_{m}} \frac{\mathbb{I}_{k_{m+1}}^{(n)}}{z-\mathbb{H}_{0}} \mathrm{~d} z,
\end{aligned}
$$

with

$$
\mathbb{I}_{k}^{(n)}= \begin{cases}\mathbb{P}_{0}^{(n)}, & k=0 \\ \mathbb{Q}_{0}^{(n)}, & k=1\end{cases}
$$

To derive the coefficients $E_{\ell}^{(n)}$ of the energy expansion in Theorem 2, we observe that

$$
\begin{aligned}
\operatorname{Tr}_{\mathcal{F}_{\perp}} \mathbb{H} \mathbb{P}^{(n)} & =\frac{1}{2 \pi \mathrm{i}} \operatorname{Tr}_{\mathcal{F}_{\perp}} \oint_{\gamma^{(n)}} \frac{\mathbb{H}}{z-\mathbb{H}} \mathrm{d} z=\frac{1}{2 \pi \mathrm{i}} \operatorname{Tr}_{\mathcal{F}_{\perp}} \oint_{\gamma^{(n)}} \frac{z}{z-\mathbb{H}} \mathrm{d} z \\
& =\delta_{0}^{(n)} E_{0}^{(n)}+\frac{1}{2 \pi \mathrm{i}} \operatorname{Tr}_{\mathcal{F}_{\perp}} \oint_{\gamma^{(n)}} \frac{z-E_{0}^{(n)}}{z-\mathbb{H}} \mathrm{d} z,
\end{aligned}
$$

then expand $(z-\mathbb{H})^{-1}$ as in Lemma 3.13 and use the residue theorem to evaluate the resulting expressions.

It remains to show that the difference

$$
\left|\operatorname{Tr}_{\mathcal{F}_{\perp}} \mathbb{A}_{N}^{(m)} \mathbb{P}^{(n)}-\sum_{\ell=0}^{a} \lambda_{N}^{\frac{\ell}{2}} \operatorname{Tr}_{\mathcal{F}_{\perp}} \mathbb{A}_{N}^{(m)} \mathbb{P}_{\ell}^{(n)}\right|
$$

is of order $\lambda_{N}^{(a+2) / 2}$. We prove this in four steps.

Step 1. First, recall that all low-energy eigenstates of $H_{N}$ exhibit condensation in $\varphi$, hence the leadingorder contribution to $\operatorname{Tr}_{\mathfrak{Y}^{N}} A^{(m)} P_{N}^{(n)}$ is determined by the condensate. To take this into account, we define the auxiliary operator

$$
\mathbb{A}_{\text {red }}^{(m)}:=\mathbb{A}_{N}^{(m)}-\langle A\rangle^{(m)} \oplus 0, \quad\langle A\rangle^{(m)}:=\left\langle\varphi^{\otimes m}, A^{(m)} \varphi^{\otimes m}\right\rangle_{\mathfrak{S}^{m}},
$$

where we have already subtracted the leading order - that is,

$$
\operatorname{Tr}_{\mathfrak{Y}^{N}} A^{(m)} P_{N}^{(n)}=\operatorname{Tr}_{\mathcal{F}_{\perp}} \mathbb{A}_{N}^{(m)} \mathbb{P}^{(n)}=\delta_{0}^{(n)}\langle A\rangle^{(m)}+\operatorname{Tr}_{\mathcal{F}_{\perp}} \mathbb{A}_{\text {red }}^{(m)} \mathbb{P}^{(n)} .
$$


Our goal is to conclude from Proposition 3.14 that

$$
\operatorname{Tr}_{\mathcal{F}_{\perp}} \mathbb{A}_{\text {red }}^{(m)} \mathbb{P}^{(n)}=\sum_{\ell=0}^{a} \lambda_{N}^{\frac{\ell}{2}} \operatorname{Tr}_{\mathcal{F}_{\perp}} \mathbb{A}_{\text {red }}^{(m)} \mathbb{P}_{\ell}^{(n)}+\mathcal{O}\left(\lambda_{N}^{\frac{a+2}{2}}\right)
$$

that is, we must show that the error terms in equation (3.52) are of the right order. Given equation (3.59), the statement of the theorem can be inferred as follows: by the definition of $\mathbb{A}_{\text {red }}^{(m)}$, equation (3.59) implies that

$$
\operatorname{Tr}_{\mathcal{F}_{\perp}} \mathbb{A}_{\text {red }}^{(m)} \mathbb{P}^{(n)}=\sum_{\ell=0}^{a} \lambda_{N}^{\frac{\ell}{2}} \operatorname{Tr}_{\mathcal{F}_{\perp}} \mathbb{A}_{N}^{(m)} \mathbb{P}_{\ell}^{(n)}-\langle A\rangle^{(m)} \sum_{\ell=0}^{a} \lambda_{N}^{\frac{\ell}{2}} \operatorname{Tr}_{\mathcal{F}_{\perp}} \mathbb{P}_{\ell}^{(n)}+\mathcal{O}\left(\lambda_{N}^{\frac{a+2}{2}}\right)
$$

Due to Proposition 3.14 and the fact that $\operatorname{Tr}_{\mathcal{F}_{\perp}} \mathbb{P}^{(n)}=\operatorname{Tr}_{\mathcal{F}_{\perp}} \mathbb{P}_{0}^{(n)}=\delta_{0}^{(n)}$ by definition, one can show that

$$
\delta_{0}^{(n)}=\operatorname{Tr}_{\mathcal{F}_{\perp}} \mathbb{P}^{(n)}=\delta_{0}^{(n)}+\sum_{\ell=1}^{a} \lambda_{N}^{\frac{\ell}{2}} \operatorname{Tr}_{\mathcal{F}_{\perp}} \mathbb{P}_{\ell}^{(n)}+\mathcal{O}\left(\lambda_{N}^{\frac{a+1}{2}}\right)
$$

for any $a \in \mathbb{N}$, which implies that $\operatorname{Tr}_{\mathcal{F}_{\perp}} \mathbb{P}_{\ell}^{(n)}=0$ for any $\ell \geq 1$. Alternatively, this can be inferred directly from the definition of $\mathbb{P}_{\ell}^{(n)}$. Hence, equation (3.60) yields

$$
\operatorname{Tr}_{\mathcal{F}_{\perp}} \mathbb{A}_{N}^{(m)} \mathbb{P}^{(n)}=\operatorname{Tr}_{\mathcal{F}_{\perp}} \mathbb{A}_{\text {red }}^{(m)} \mathbb{P}^{(n)}+\delta_{0}^{(n)}\langle A\rangle^{(m)}=\sum_{\ell=0}^{a} \lambda_{N}^{\frac{\ell}{2}} \operatorname{Tr}_{\mathcal{F}_{\perp}} \mathbb{A}_{N}^{(m)} \mathbb{P}_{\ell}^{(n)}+\mathcal{O}\left(\lambda_{N}^{\frac{a+2}{2}}\right)
$$

It remains to prove the two estimates (3.59) and (3.61). To deal with both problems simultaneously, let us consider

$$
\mathbb{A} \in\left\{\mathbb{A}_{\text {red }}^{(m)}, \mathbb{1}\right\}
$$

Step 2. First we show that $\mathbb{A}$ satisfies an estimate of the form

$$
\|\mathbb{A} \phi\|_{\mathcal{F}_{\perp}} \leq \mathfrak{C} N^{\alpha}\left(\left\|\left(\mathcal{N}_{\perp}+1\right) \phi\right\|_{\mathcal{F}_{\perp}}+\left\|\mathbb{H} \mathbb{H}_{0} \phi\right\|_{\mathcal{F}_{\perp}}\right) .
$$

For $\mathbb{A}=\mathbb{1}$, this holds trivially with $\alpha=0$; for $\mathbb{A}=\mathbb{A}_{\text {red }}^{(m)}$, we prove the bound (3.63) with $\alpha=-\frac{1}{2}$ (Lemma 5.4). Let us explain the main idea of the proof for the simplest case $m=1$. First, we use $U_{N, \varphi}$ to reduce the problem to an estimate on $\mathfrak{S}^{N}$ and insert identities $1=p_{1}+q_{1}$ (see definition (2.18)),

$$
\left\|\mathbb{A}_{\text {red }}^{(1)} \phi\right\|_{\mathcal{F}_{\perp}}=\|\left(p_{1} A_{1}^{(1)} p_{1}-\langle A\rangle^{(1)}+\left(q_{1} A_{1}^{(1)} p_{1}+\text { h.c. }\right)+q_{1} A_{1}^{(1)} q_{1}\right) U_{N, \varphi}^{*} \phi \|_{\mathfrak{S}^{N}}
$$

for any $\phi \in \mathcal{F}_{\perp}^{\leq N} \oplus 0$. For the first term, one observes that

$$
p_{1} A_{1}^{(1)} p_{1}-\langle A\rangle^{(1)}=-q_{1}\langle A\rangle^{(1)}
$$

hence every contribution to equation (3.64) contains at least one projection $q$ onto the orthogonal complement of the condensate wave function. This gives a prefactor $N^{-1 / 2}$ because

$$
\left\|q_{1} U_{N, \varphi}^{*} \phi\right\|_{\mathfrak{S}^{N}}=N^{-\frac{1}{2}}\left\|\mathrm{~d} \Gamma_{\perp}(q)^{\frac{1}{2}} U_{N, \varphi}^{*} \phi\right\|_{\mathfrak{S}^{N}}=N^{-\frac{1}{2}}\left\|\mathcal{N}_{\perp}^{\frac{1}{2}} \phi\right\|_{\mathcal{F}_{\perp}^{\leq N}} .
$$


To control the action of $A^{(1)}$ on $U_{N, \varphi}^{*} \phi$, note that $A^{(1)}$ is relatively bounded by $h$ by assumption, and, for any $\psi_{N} \in \mathfrak{H}_{\mathrm{sym}}^{N}$,

$$
\begin{aligned}
\left\|h_{1} \psi_{N}\right\|_{\mathfrak{S}^{N}}^{2} & =N^{-1} \sum_{j=1}^{N}\left\langle\psi_{N}, h_{j} h_{j} \psi_{N}\right\rangle_{\mathfrak{H}^{N}} \\
& \leq N^{-1} \sum_{1 \leq j, \ell \leq N}\left\langle\psi_{N}, h_{j} h_{\ell} \psi_{N}\right\rangle_{\mathfrak{H}^{N}}=N^{-1}\left\|\mathbb{K}_{0} \psi_{N}\right\|_{\mathfrak{H}^{N}}^{2}
\end{aligned}
$$

by permutation symmetry of $\psi_{N}$ and as $h \geq 0$. The full argument is given in Section 5.2.2.

Step 3. Proposition 3.14 implies that

$$
\operatorname{Tr}_{\mathcal{F}_{\perp}} \mathbb{A P}^{(n)}-\sum_{\ell=0}^{a} \lambda_{N}^{\frac{\ell}{2}} \operatorname{Tr}_{\mathcal{F}_{\perp}} \mathbb{A P}_{\ell}^{(n)}=\lambda_{N}^{\frac{a+1}{2}}\left(\operatorname{Tr}_{\mathcal{F}_{\perp}} \mathbb{A B}_{P}^{(n)}(a)+\operatorname{Tr}_{\mathcal{F}_{\perp}} \mathbb{A B}_{Q}^{(n)}(a)\right),
$$

with $\mathbb{B}_{P}^{(n)}$ and $\mathbb{B}_{Q}^{(n)}$ as defined in equations (3.53) and (3.54). Let us sketch the estimate of the remainders for the leading order $a=0$ and the simplest case of a nondegenerate eigenvalue of $\mathbb{H}_{0}$ (and thus $\mathbb{H}$ ). In this case,

$$
\begin{aligned}
& \operatorname{Tr}_{\mathcal{F}_{\perp}} \mathbb{A B}_{Q}^{(n)}(0)=\frac{1}{2 \pi \mathrm{i}} \operatorname{Tr}_{\mathcal{F}_{\perp}} \oint_{\gamma^{(n)}} \frac{1}{z-E_{0}^{(n)}} \frac{\mathbb{Q}^{(n)}}{z-\mathbb{H}<} \mathbb{R}_{0} \mathbb{P}_{0}^{(n)} \mathbb{A} \mathrm{d} z, \\
& \operatorname{Tr}_{\mathcal{F}_{\perp}} \mathbb{A B}_{P}^{(n)}(0)=\frac{1}{2 \pi \mathrm{i}} \operatorname{Tr}_{\mathcal{F}_{\perp}} \oint_{\gamma^{(n)}} \frac{\mathbb{P}^{(n)}}{z-E^{(n)}} \mathbb{R}_{0} \frac{1}{z-\mathbb{H}_{0}} \mathbb{A} \mathrm{d} z,
\end{aligned}
$$

both of which contain at least one rank 1 projection. By construction, the circumference of $\gamma^{(n)}$ and its distance to $E^{(n)}$ and $E_{0}^{(n)}$ are of order 1. Hence, after interchanging trace and integral, it remains to control

$$
\begin{aligned}
& \left\|\left.\left\langle\chi_{0}^{(n)}, \mathbb{A} \frac{\mathbb{Q}^{(n)}}{z-\mathbb{H}^{<}} \mathbb{R}_{0} \chi_{0}^{(n)}\right\rangle\right|_{\mathcal{F}_{\perp}} \leq\right\| \mathbb{A}_{0}^{(n)}\left\|_{\mathcal{F}_{\perp}}\right\| \frac{\mathbb{Q}^{(n)}}{z-\mathbb{H}^{<}}\left\|_{\text {op }}\right\| \mathbb{R}_{0} \chi_{0}^{(n)} \|_{\mathcal{F}_{\perp}}, \\
& \left\|\left.\left\langle\chi^{(n)}, \mathbb{R}_{0} \frac{1}{z-\mathbb{H}_{0}} \mathbb{A} \chi^{(n)}\right\rangle\right|_{\mathcal{F}_{\perp}} \leq\right\| \chi^{(n)}\left\|_{\mathcal{F}_{\perp}}\right\| \mathbb{A} \frac{1}{z-\mathbb{H}_{0}} \mathbb{R}_{0} \chi^{(n)} \|_{\mathcal{F}_{\perp}}
\end{aligned}
$$

for $z \in \gamma^{(n)}$. To estimate these expressions, recall that $\mathbb{R}_{0}$ is constructed out of the operators $\mathbb{K}_{j}$ from definition (2.41) and the Taylor remainders in Lemma 3.11. By inequalities (2.5) and (2.6), $\mathbb{K}_{1}$ to $\mathbb{K}_{3}$ are bounded by powers of $\left(\mathcal{N}_{\perp}+1\right)$. Concerning $\mathbb{K}_{4}$, note that it can be written as

$$
\mathbb{K}_{4}=\mathrm{d} \Gamma_{\perp}(v)+\mathrm{d} \Gamma_{\perp}\left(v * \varphi^{2} \otimes \mathbb{1}+\mathbb{1} \otimes v * \varphi^{2}+\mathbb{1} \otimes \mathbb{1}\left\langle\varphi, v * \varphi^{2} \varphi\right\rangle\right) .
$$

Whereas the second term can be controlled by powers of $\left(\mathcal{N}_{\perp}+1\right)$, this is not true for $\mathrm{d} \Gamma_{\perp}(v)$, since $v$ may be unbounded. However, due to the estimate (2.3), it can be bounded in terms of $\mathbb{K}_{0}^{1 / 2}$ and $\left(\mathcal{N}_{\perp}+1\right)$ (Lemma 5.2). In summary, we find (see Lemma 5.3) that

$$
\left\|\mathbb{R}_{0} \chi_{0}^{(n)}\right\|_{\mathcal{F}_{\perp}} \leq \mathbb{C}\left(\left\|\left(\mathcal{N}_{\perp}+1\right)^{2} \chi_{0}^{(n)}\right\|+\left\|\left(\mathcal{N}_{\perp}+1\right)^{\frac{3}{2}} \mathbb{H}_{0} \chi_{0}^{(n)}\right\|\right) \leq \mathfrak{C}(n),
$$

because $\left\|\left(\mathcal{N}_{\perp}+1\right)^{\frac{3}{2}} \mathbb{H}_{0} \chi_{0}^{(n)}\right\| \leq \mathfrak{C}\left\|\left(\mathcal{N}_{\perp}+1\right)^{\frac{3}{2}} \chi_{0}^{(n)}\right\|$ and because finite moments of $\mathcal{N}_{\perp}$ with respect to $\chi_{0}^{(n)}$ are bounded uniformly in $N$ (Lemma 4.7d). Analogously, the estimate (3.63) yields

$$
(3.70 a) \leq \mathfrak{C}(n) N^{\alpha},
$$


with $\alpha=-1 / 2$ for $\mathbb{A}=\mathbb{A}_{\text {red }}^{(m)}$ and $\alpha=0$ for $\mathbb{A}=\mathbb{1}$. Moreover,

$$
(3.70 b) \leq \mathfrak{C} N^{\alpha}\left\|\left(\mathcal{N}_{\perp}+1\right) \frac{1}{z-\mathbb{H}_{0}} \mathbb{R}_{0} \chi^{(n)}\right\|_{\mathcal{F}_{\perp}} \leq \mathfrak{C} N^{\alpha}\left\|\mathbb{R}_{0} \chi^{(n)}\right\|_{\mathcal{F}_{\perp}} .
$$

The last inequality, which is proven in Lemma 5.5, follows essentially from the observation that $\mathcal{N}_{\perp} \leq$ $\mathfrak{C} \mathbb{U}_{\mathcal{V}_{0}}\left(\mathbb{H}_{0}-E_{0}^{(0)}+1\right) \mathbb{U}_{\mathcal{V}_{0}}^{*}$, for $\mathbb{U}_{\mathcal{V}_{0}}$ the Bogoliubov transformation diagonalising $\mathbb{H}_{0}$ (Lemma 4.7e), because one can control the action of $\mathbb{U}_{\mathcal{V}_{0}}$ on the number operator (Lemma 4.4) sufficiently well. As opposed to the estimate (3.70a), we do not a priori know this to be of order $N^{\alpha}$, since we do not have sufficient control of $\left(\mathcal{N}_{\perp}+1\right)^{b} \chi^{(n)}$ for $b>1 / 2$ and of $\mathbb{R}_{0} \chi^{(n)}$, which contains a contribution $\mathbb{K}_{4} \chi^{(n)}$.

Step 4. To prove a uniform bound for $\operatorname{Tr}_{\mathcal{F}_{\perp}}\left(\mathcal{N}_{\perp}+1\right)^{b} \mathbb{P}^{(n)}$ for any $b \geq 1$, we make use of the a priori bound

$$
\begin{aligned}
& \operatorname{Tr}_{\mathcal{F}_{\perp}}\left(\mathcal{N}_{\perp}+1\right) \mathbb{P}^{(n)} \leq \mathfrak{C}(n), \\
& \operatorname{Tr}_{\mathcal{F}_{\perp}}\left(\mathcal{N}_{\perp}+1\right)^{b} \mathbb{P}^{(n)} \leq \mathfrak{C}(b, n) N^{\frac{1}{3}} \operatorname{Tr}_{\mathcal{F}_{\perp}}\left(\mathcal{N}_{\perp}+1\right)^{b-1} \mathbb{P}^{(n)}
\end{aligned}
$$

(Lemma 4.8c) to close a bootstrap argument. Let us explain the strategy for the simplest case $b=2$ and a nondegenerate eigenvalue $E_{0}^{(n)}$. First, we expand $\mathbb{P}^{(n)}$ one step around $\mathbb{P}_{0}^{(n)}$ - that is, we apply equation (3.68) to $\mathbb{A}=\left(\mathcal{N}_{\perp}+1\right)^{2}$ for $a=0$. Since $\operatorname{Tr}_{\mathcal{F}_{\perp}}\left(\mathcal{N}_{\perp}+1\right)^{2} \mathbb{P}_{0}^{(n)}$ is bounded uniformly in $N$, it remains to show that the error terms corresponding to expressions (3.70a) and (3.70b) are bounded. Whereas expression (3.70a) is clearly bounded uniformly in $N$, we make use of the foregoing a priori bound to estimate expression (3.70b). The positive powers of $N$ arising from this can be compensated for by the prefactor $\lambda_{N}^{1 / 2}$ in equation (3.68) - which, however, requires some manipulations since we do not yet have a sufficient bound for $\mathbb{K}_{4} \chi^{(n)}$. This cancellation is precisely the point where the restriction $\varepsilon(N) \leq C N^{\frac{1}{3}}$ in Assumption 3 enters. The full argument is given in Lemma 5.6. Note that for the $d$ dimensional torus, a uniform bound for $\operatorname{Tr}_{\mathcal{F}_{\perp}}\left(\mathcal{N}_{\perp}+1\right)^{b}$ was shown in [44, Corollary 3.2] by a different argument.

Finally, the estimate $\operatorname{Tr}_{\mathcal{F}_{\perp}} \mathbb{K}_{4}^{2} \mathbb{P}^{(n)} \leq \mathfrak{C}$ follows from a similar bootstrap argument, using the a priori bound

$$
\mathbb{K}_{4} \leq \mathfrak{C}\left(\left(\mathcal{N}_{\perp}+1\right)^{\frac{3}{2}} \mathrm{~d} \Gamma_{\perp}(h)\left(\mathcal{N}_{\perp}+1\right)^{\frac{3}{2}}+\left(\mathcal{N}_{\perp}+1\right)^{4}\right)
$$

together with Assumption 3 and the previous estimate of $\operatorname{Tr}_{\mathcal{F}_{\perp}}\left(\mathcal{N}_{\perp}+1\right)^{b} \mathbb{P}(n)$.

Remark 3.15. For interactions $v \in L^{\infty}\left(\mathbb{R}^{d}\right)$, step 4 is not necessary. In this case, Assumption 3 holds with $\varepsilon(N)=\mathcal{O}(1)$ [27, Lemma 1], hence the a priori bound (3.75) is already uniform in $N$ (see Lemma 4.8c), and moreover, $\mathbb{K}_{4}$ is bounded by powers of $\mathcal{N}_{\perp}$.

The latter also explains why the estimate of the growth of $\mathfrak{C}(n, m, a)$ in $a$ is better than for generic $v$ (Remark 3.5): since all operators $\mathbb{H}_{j}$ and $\mathbb{R}_{j}$ from the expansion of $\mathbb{H}^{<}$are bounded by powers of $\mathcal{N}_{\perp}$ (and not by $\mathbb{H}_{0}$ ), each commuting with a resolvent $\left(z-\mathbb{H}_{0}\right)^{-1}$ cancels one of these powers as in the estimate (3.74). Consequently, the final power of $\mathcal{N}_{\perp}$ acting on $\chi^{(n)}$ and $\chi_{0}^{(n)}$ is less than in the generic case, where this effect is cancelled by $\mathbb{H}_{0}$ hitting the resolvent. Since conjugating powers of $\mathcal{N}_{\perp}$ with Bogoliubov transformations is the main source for the growth in $a$ (see Lemma 4.4), this leads to a better estimate.

\section{Bogoliubov theory}

In this section, we summarise some known results concerning the Bogoliubov Hamiltonian $\mathbb{H}_{0}$ and its connection to the $N$-body Hamiltonian $H_{N}$. As preparation, recall that

$$
a_{x}^{\dagger} F\left(\mathcal{N}_{\perp}\right)=F\left(\mathcal{N}_{\perp}-1\right) a_{x}^{\dagger}, \quad a_{x} F\left(\mathcal{N}_{\perp}\right)=F\left(\mathcal{N}_{\perp}+1\right) a_{x}
$$

for any function $F$. Moreover, normal ordered expressions can be bounded in terms of $\mathcal{N}_{\perp}$ : 
Lemma 4.1. Let $n, p \geq 0$ and let $f: \mathfrak{H}_{\perp}^{p} \rightarrow \mathfrak{H}_{\perp}^{n}$ be a bounded operator with (Schwartz) kernel $f\left(x^{(n)} ; y^{(p)}\right)$ and $\phi \in \mathcal{F}_{\perp}$. Then

$$
\left\|\int \mathrm{d} x^{(n)} \mathrm{d} y^{(p)} f\left(x^{(n)} ; y^{(p)}\right) a_{x_{1}}^{\dagger} \cdots a_{x_{n}}^{\dagger} a_{y_{1}} \cdots a_{y_{p}} \phi\right\|_{\mathcal{F}_{\perp}} \leq\|f\|_{\mathfrak{G}_{\perp}^{p} \rightarrow \mathfrak{H}_{\perp}^{n}}\left\|\left(\mathcal{N}_{\perp}+n\right)^{\frac{n+p}{2}} \phi\right\|_{\mathcal{F}_{\perp}} .
$$

A proof is given in [11, Lemma 5.1]. In the following, we will always assume that Assumptions 1, 2 and 3 are satisfied.

\subsection{Bogoliubov transformations}

We begin by briefly recalling the concept of Bogoliubov transformations, mainly following [67, 11]. Let us consider

$$
F=f \oplus J g=f \oplus \bar{g}=\left(\begin{array}{l}
f \\
\bar{g}
\end{array}\right) \in \mathfrak{H}_{\perp} \oplus \mathfrak{H}_{\perp},
$$

where $J: \mathfrak{H}_{\perp} \rightarrow \mathfrak{H}_{\perp},(J f)(x)=\overline{f(x)}$, denotes complex conjugation, and define the generalised creation and annihilation operators $A(F)$ and $A^{\dagger}(F)$ as

$$
A(F)=a(f)+a^{\dagger}(g), \quad A^{\dagger}(F)=A(\mathcal{J} F)=a^{\dagger}(f)+a(g)
$$

for $\mathcal{J}=\left(\begin{array}{ll}0 & J \\ J & 0\end{array}\right)$. An operator $\mathcal{V}$ on $\mathfrak{H}_{\perp} \oplus \mathfrak{H}_{\perp}$ such that $F \mapsto A(\mathcal{V} F)$ has the same properties as $F \mapsto A(F)$, that is,

$$
A^{\dagger}(\mathcal{V} F)=A(\mathcal{V} \mathcal{J} F), \quad\left[A\left(\mathcal{V} F_{1}\right), A^{\dagger}\left(\mathcal{V} F_{2}\right)\right]=\left[A\left(F_{1}\right), A^{\dagger}\left(F_{2}\right)\right]
$$

is called a (bosonic) Bogoliubov map.

Definition 4.2. A bounded operator $\mathcal{V}: \mathfrak{H}_{\perp} \oplus \mathfrak{H}_{\perp} \rightarrow \mathfrak{H}_{\perp} \oplus \mathfrak{H}_{\perp}$ is a Bogoliubov map if

$$
\mathcal{V}^{*} \mathcal{S} \mathcal{V}=\mathcal{S}=\mathcal{V} S \mathcal{V}^{*}, \quad \mathcal{J V} \mathcal{J}=\mathcal{V},
$$

for $\mathcal{S}=\left(\begin{array}{cc}1 & 0 \\ 0 & -1\end{array}\right)$. Equivalently, $\mathcal{V}$ has the block form

$$
\mathcal{V}:=\left(\begin{array}{ll}
U & \bar{V} \\
V & \bar{U}
\end{array}\right), \quad U, V: \mathfrak{H}_{\perp} \rightarrow \mathfrak{H}_{\perp},
$$

where $U$ and $V$ satisfy the relations

$$
U^{*} U=\mathbb{1}+V^{*} V, \quad U U^{*}=\mathbb{1}+\overline{V V}^{*}, \quad V^{*} \bar{U}=U^{*} \bar{V}, \quad U V^{*}=\overline{V U}^{*} .
$$

We denote the set of Bogoliubov maps on $\mathfrak{H}_{\perp} \oplus \mathfrak{H}_{\perp}$ as

$$
\mathfrak{B}\left(\mathfrak{H}_{\perp}\right):=\left\{\mathcal{V} \in \mathcal{L}\left(\mathfrak{H}_{\perp} \oplus \mathfrak{H}_{\perp}\right) \mid \mathcal{V} \text { is a Bogoliubov map }\right\} .
$$

The adjoint and inverse of $\mathcal{V} \in \mathfrak{B}\left(\mathfrak{H}_{\perp}\right)$ with block form (4.7) are given as

$$
\mathcal{V}^{*}=\left(\begin{array}{ll}
U^{*} & V^{*} \\
\bar{V}^{*} & \bar{U}^{*}
\end{array}\right), \quad \mathcal{V}^{-1}=\mathcal{S} \mathcal{V}^{*} \mathcal{S}=\left(\begin{array}{cc}
U^{*} & -V^{*} \\
-\bar{V}^{*} & \bar{U}^{*}
\end{array}\right) .
$$

Under certain conditions, Bogoliubov maps can be unitarily implemented on $\mathcal{F}_{\perp}$ (see, e.g., [67, Theorem 9.5]): 
Lemma 4.3. Let $\mathcal{V} \in \mathfrak{B}\left(\mathfrak{H}_{\perp}\right)$. Then there exists a unitary transformation $\mathbb{U}_{\mathcal{V}}: \mathcal{F}_{\perp} \rightarrow \mathcal{F}_{\perp}$ such that

$$
\mathbb{U}_{\mathcal{V}} A(F) \mathbb{U}_{\mathcal{V}}^{*}=A(\mathcal{V} F)
$$

for all $F \in \mathfrak{H}_{\perp} \oplus \mathfrak{H}_{\perp}$ if and only if

$$
\|V\|_{H S\left(\mathfrak{H}_{\perp}\right)}^{2}:=\operatorname{Tr}_{\mathfrak{H}_{\perp}}\left(V^{*} V\right)<\infty
$$

(Shale-Stinespring condition). In this case, $v$ is called (unitarily) implementable. We refer to the unitary implementation of a Bogoliubov map as Bogoliubov transformation.

If $V$ is Hilbert-Schmidt, the map $\mathcal{V} \mapsto \mathbb{U}_{\mathcal{V}}$ is a group homomorphism, which in particular implies that

$$
U_{\mathcal{V}^{-1}}=\left(\mathbb{U}_{\mathcal{V}}\right)^{-1}=\mathbb{U}_{\mathcal{V}}^{*}
$$

Writing $U, V$ as integral operators with (Schwartz) kernels $U(x ; y)$ and $V(x ; y)$, that is,

$$
(U f)(x)=\int U(x ; y) f(y) \mathrm{d} y, \quad(V f)(x)=\int V(x ; y) f(y) \mathrm{d} y
$$

for any $f \in \mathfrak{H}_{\perp}$, we can express the transformation rule (4.11) as

$$
\begin{aligned}
& \mathbb{U}_{\mathcal{V}} a_{x} \mathbb{U}_{\mathcal{V}}^{*}=\int \mathrm{d} y \overline{U(y ; x)} a_{y}+\int \mathrm{d} y \overline{V(y ; x)} a_{y}^{\dagger}, \\
& \mathbb{U}_{\mathcal{V}} a_{x}^{\dagger} \mathbb{U}_{\mathcal{V}}^{*}=\int \mathrm{d} y V(y ; x) a_{y}+\int \mathrm{d} y U(y ; x) a_{y}^{\dagger} .
\end{aligned}
$$

In particular, powers of $\mathcal{N}_{\perp}$ conjugated with $\mathbb{U}_{\mathcal{V}}$ can be bound as follows (see [11, Lemma 4.4] for a proof):

Lemma 4.4. Let $\mathcal{V} \in \mathfrak{B}\left(\mathfrak{H}_{\perp}\right)$ be unitarily implementable and denote by $\mathbb{U}_{\mathcal{V}}$ the corresponding Bogoliubov transformation on $\mathcal{F}_{\perp}$. Then it holds for any $b \in \mathbb{N}$ that

$$
\mathbb{U}_{\mathcal{V}}\left(\mathcal{N}_{\perp}+1\right)^{b} \mathbb{U}_{\mathcal{V}}^{*} \leq C_{\mathcal{V}}^{b} b^{b}\left(\mathcal{N}_{\perp}+1\right)^{b}
$$

in the sense of operators on $\mathcal{F}_{\perp}$, where

$$
C_{V}:=2\|V\|_{H S}^{2}+\|U\|_{o p}^{2}+1
$$

for $\mathcal{V}=\left(\begin{array}{ll}U & \bar{V} \\ V & \bar{U}\end{array}\right)$ and with $\|\cdot\|_{o p}:=\|\cdot\|_{\mathcal{L}\left(\mathfrak{H}_{\perp}\right)}$ and $\|\cdot\|_{H S}:=\|\cdot\|_{H S\left(\mathfrak{H}_{\perp}\right)}$.

Finally, we recall the notion of quasi-free states:

Definition 4.5. A normalised state $\phi \in \mathcal{F}_{\perp}$ is called a quasi-free (pure) state if there exists some $\nu \in \mathfrak{B}\left(\mathfrak{H}_{\perp}\right)$ such that

$$
\phi=\mathbb{U}_{\mathcal{V}}|\Omega\rangle
$$

Alternatively, quasi-free states can be defined via Wick's rule (e.g. [46, Theorem 1.6]):

Lemma 4.6. Let $\phi \in \mathcal{F}_{\perp}$ be normalised. Then $\phi$ is quasi-free if and only if

$$
\langle\phi, \mathcal{N} \phi\rangle_{\mathcal{F}_{\perp}}<\infty
$$


and

$$
\begin{aligned}
\left\langle\boldsymbol{\phi}, a^{\sharp}\left(f_{1}\right) \cdots a^{\sharp}\left(f_{2 n-1}\right) \boldsymbol{\phi}\right\rangle_{\mathcal{F}_{\perp}} & =0, \\
\left\langle\boldsymbol{\phi}, a^{\sharp}\left(f_{1}\right) \cdots a^{\sharp}\left(f_{2 n}\right) \boldsymbol{\phi}\right\rangle_{\mathcal{F}_{\perp}} & =\sum_{\sigma \in P_{2 n}} \prod_{j=1}^{n}\left\langle\boldsymbol{\phi}, a^{\sharp}\left(f_{\sigma(2 j-1)}\right) a^{\sharp}\left(f_{\sigma(2 j)}\right) \boldsymbol{\phi}\right\rangle_{\mathcal{F}_{\perp}}
\end{aligned}
$$

for $a^{\sharp} \in\left\{a^{\dagger}, a\right\}, n \in \mathbb{N}$ and $f_{1}, \ldots, f_{2 n} \in \mathfrak{H}_{\perp}$. Here, $P_{2 n}$ denotes the set of pairings

$$
P_{2 n}:=\left\{\sigma \in \mathfrak{S}_{2 n}: \sigma(2 a-1)<\min \{\sigma(2 a), \sigma(2 a+1)\} \forall a \in\{1,2, \ldots, 2 n\}\right\},
$$

where $\mathfrak{S}_{2 n}$ denotes the symmetric group on the set $\{1,2, \ldots, 2 n\}$.

\subsection{Properties of $\mathbb{H}$ and $\mathbb{H}_{0}$}

Since $\mathbb{H}_{0}$ is a quadratic Hamiltonian, it can be diagonalised by Bogoliubov transformations, which makes it possible to compute its spectrum:

\section{Lemma 4.7.}

(a) There exists a unitarily implementable Bogoliubov map

$$
\mathcal{V}_{0}=\left(\begin{array}{ll}
U_{0} & \bar{V}_{0} \\
V_{0} & \bar{U}_{0}
\end{array}\right) \in \mathfrak{B}\left(\mathfrak{G}_{\perp}\right)
$$

such that the corresponding Bogoliubov transformation $\mathbb{U}_{\mathcal{V}_{0}}: \mathcal{F}_{\perp} \rightarrow \mathcal{F}_{\perp}$ diagonalises $\mathbb{H}_{0}-$ that is, there exists a self-adjoint operator $D>0$ on $\mathfrak{H}_{\perp}$ such that

$$
\mathbb{U}_{\mathcal{V}_{0}} \mathbb{H}_{0} \mathbb{U}_{\mathcal{V}_{0}}^{*}=\mathrm{d} \Gamma_{\perp}(D)+\inf \sigma\left(\mathbb{H}_{0}\right)
$$

The spectrum of $D$ is purely discrete, and we denote its eigenvalues as

$$
0<d^{(0)}<d^{(1)}<\cdots<d^{(j)}<\cdots .
$$

In particular, D admits a complete set of normalised eigenfunctions, denoted as $\left\{\xi_{j}\right\}_{j \geq 0}$.

(b) The spectrum of $\mathbb{H}_{0}$ is purely discrete, and the ground-state energy of $\mathbb{H}_{0}$ is negative. For any $n \in \mathbb{N}$, there exists some $k \in \mathbb{N}_{0}$ and some tuple $\left(v_{0}, \ldots, v_{k}\right) \in \mathbb{N}_{0}^{k+1}$ such that

$$
E_{0}^{(n)}=E_{0}^{(0)}+v_{0} d^{(0)}+v_{1} d^{(1)}+\cdots+v_{k} d^{(k)} .
$$

Further, $\mathfrak{g}^{(n)}>0$, for $\mathfrak{g}^{(n)}$ as in definition (3.33).

(c) The ground state of $\mathbb{H}_{0}$ is unique and given by

$$
\chi_{0}^{(0)}=\mathbb{U}_{\mathcal{V}_{0}}^{*}|\Omega\rangle
$$

For each $n \in \mathbb{N}$, there exists a basis $\left\{\chi_{0}^{(n, m)}\right\}_{1 \leq m \leq \delta_{0}^{(n)}}$ of $\mathfrak{E}_{0}^{(n)}$ such that

$$
\chi_{0}^{(n, m)}=\mathbb{U}_{\mathcal{V}_{0}}^{*} \frac{\left(a^{\dagger}\left(\xi_{0}\right)\right)^{v_{0}}}{\sqrt{v_{0} !}} \frac{\left(a^{\dagger}\left(\xi_{1}\right)\right)^{v_{1}}}{\sqrt{v_{1} !}} \cdots \frac{\left(a^{\dagger}\left(\xi_{k}\right)\right)^{v_{k}}}{\sqrt{v_{k} !}}|\Omega\rangle
$$

for some $k \in \mathbb{N}_{0}$ and some tuple $\left(v_{0}, \ldots, v_{k}\right) \in \mathbb{N}_{0}^{k+1}$ depending on $m$. 
(d) Let $b \in \mathbb{N}_{0}$ and let $\chi_{0}^{(n, m)} \in \mathfrak{E}_{0}^{(n)}$ be given by equation (4.25). Then

$$
\left\langle\chi_{0}^{(n, m)},\left(\mathcal{N}_{\perp}+1\right)^{b} \chi_{0}^{(n, m)}\right\rangle_{\mathcal{F}_{\perp}} \leq\left(\mathfrak{C} b\left(1+v_{0}+\cdots+v_{k}\right)\right)^{b} \leq(\mathfrak{C}(n) b)^{b},
$$

and

$$
\left\|\left(\mathcal{N}_{\perp}+1\right)^{b} \mathbb{P}_{0}^{(n)}\right\|_{\mathcal{L}\left(\mathcal{F}_{\perp}\right)} \leq(\mathfrak{C}(n) b)^{b} .
$$

(e) In the sense of operators on $\mathcal{F}_{\perp}$, it holds that

$$
\mathcal{N}_{\perp}+1 \leq \mathfrak{C} \mathbb{U}_{\mathcal{V}_{0}}^{*}\left(\mathcal{N}_{\perp}+1\right) \mathbb{U}_{\mathcal{V}_{0}} \leq \mathfrak{C}\left(\mathbb{H}_{0}-E_{0}^{(0)}+1\right)
$$

All statements of Lemma 4.7 are well known and are proven for various models in, for example, [67, $47,36,53,56]$. In the following, we summarise a proof for our model:

Proof. Part (a). Let us abbreviate $\widetilde{K}:=q K q$ for $K$ as in definition (2.16). By Lemma 2.2, $\widetilde{K}(h+\widetilde{K})^{-1}$ is Hilbert-Schmidt on $\mathfrak{H}_{\perp}$, since

$$
\left\|\widetilde{K}(h+\widetilde{K})^{-1}\right\|_{\mathrm{HS}} \leq\|K\|_{\mathrm{HS}}\left\|(h+\widetilde{K})^{-1}\right\|_{\mathrm{op}} \leq g_{\mathrm{H}}^{-1}\|K\|_{\mathrm{HS}}
$$

as $K \geq 0$ and $h \geq g_{\mathrm{H}}>0$ on $\mathfrak{H}_{\perp}$. Moreover, $G:=(h+\widetilde{K})^{-\frac{1}{2}} \widetilde{K}(h+\widetilde{K})^{-\frac{1}{2}}$ is Hilbert-Schmidt on $\mathfrak{H}_{\perp}$, since

$$
\operatorname{Tr}\left(G^{*} G\right)=\operatorname{Tr}\left(\left(\widetilde{K}(h+\widetilde{K})^{-1}\right)^{2}\right) \leq\left\|\widetilde{K}(h+\widetilde{K})^{-1}\right\|_{\mathrm{HS}}^{2},
$$

and $\|G\|_{\text {op }}=\left\|\widetilde{K}^{\frac{1}{2}}(h+\widetilde{K})^{-1} \widetilde{K}^{\frac{1}{2}}\right\|_{\text {op }}<1$ because

$$
\widetilde{K}^{\frac{1}{2}}(h+\widetilde{K})^{-1} \widetilde{K}^{\frac{1}{2}} \leq \frac{\widetilde{K}}{g_{\mathrm{H}}+\widetilde{K}} \leq \frac{\|\widetilde{K}\|_{\mathrm{op}}}{g_{\mathrm{H}}+\|\widetilde{K}\|_{\text {op }}} \mathbb{1},
$$

where we use the fact that the inverse is operator monotone and that $x \mapsto x\left(g_{\mathrm{H}}+x\right)^{-1}$ is increasing. Hence, by [53, Theorems 1 and 2], there exists a unitarily implementable $\mathcal{V}_{0} \in \mathfrak{B}\left(\mathfrak{H}_{\perp}\right)$ such that

$$
\mathcal{V}_{0} \mathcal{A} \mathcal{V}_{0}^{*}=\mathcal{V}_{0}\left(\begin{array}{cc}
h+\widetilde{K} & \widetilde{K} \\
\widetilde{K} & h+\widetilde{K}
\end{array}\right) \mathcal{V}_{0}^{*}=\left(\begin{array}{cc}
D & 0 \\
0 & J D J
\end{array}\right)
$$

for some self-adjoint operator $D>0$ on $\mathfrak{H}_{\perp}$, and

$$
\mathbb{U}_{\mathcal{V}_{0}} \mathbb{H}_{0} \mathbb{U}_{\mathcal{V}_{0}}^{*}=\mathrm{d} \Gamma_{\perp}(D)+\inf \sigma\left(\mathbb{H}_{0}\right),
$$

where $\mathbb{U}_{\mathcal{V}_{0}}$ denotes the unitary implementation of $\mathcal{V}_{0}$ on $\mathcal{F}_{\perp}$. Finally, one can show as in Step 6 in the proof of [36, Theorem A.1] that $D$ has purely discrete spectrum.

Parts (b) and (c). By [36, Theorem A.1(iii-iv)], $\sigma\left(\mathbb{H}_{0}\right)=\sigma_{\text {disc }}\left(\mathbb{H}_{0}\right)$ and inf $\sigma\left(\mathbb{H}_{0}\right)<0$. Since $D>0$, $|\Omega\rangle$ is the unique ground state of $\mathrm{d} \Gamma_{\perp}(D)$ with eigenvalue 0 , hence $\mathbb{U}_{\mathcal{V}_{0}}^{*}|\Omega\rangle$ is the unique ground state 
of $\mathbb{H}_{0}$ with eigenvalue $E_{0}^{(0)}=\inf \sigma\left(\mathbb{H}_{0}\right)$ by equation (4.21). By part (a), there is a complete set of normalised eigenstates $\left\{\xi_{j}\right\}_{j \geq 0}$ for $D$, hence

$$
\mathrm{d} \Gamma_{\perp}(D)=\sum_{j \geq 0} d^{(j)} a^{\dagger}\left(\xi_{j}\right) a\left(\xi_{j}\right)
$$

Consequently, all eigenstates of $\mathrm{d} \Gamma_{\perp}(D)$ can be written as

$$
\frac{\left(a^{\dagger}\left(\xi_{0}\right)\right)^{v_{0}}}{\sqrt{v_{0} !}} \cdots \frac{\left(a^{\dagger}\left(\xi_{k}\right)\right)^{v_{k}}}{\sqrt{v_{k} !}}|\Omega\rangle
$$

for some $k \in \mathbb{N}_{0}$, and all eigenvalues of $\mathrm{d} \Gamma_{\perp}(D)$ are of the form

$$
v_{0} d^{(0)}+v_{1} d^{(1)}+\cdots+v_{k} d^{(k)}
$$

for some $k \in \mathbb{N}_{0}$ and $\left(v_{0}, \ldots, v_{k}\right) \in \mathbb{N}_{0}^{k+1}$. Finally, equations (4.25) and (4.23) follow from equation (4.21).

Part (d). For $\chi_{0}^{(n, m)}$ as in equation (4.25), we compute by Lemma 4.4 that

$$
\begin{gathered}
\left\langle\chi_{0}^{(n, m)},\left(\mathcal{N}_{\perp}+1\right)^{b} \chi_{0}^{(n, m)}\right\rangle_{\mathcal{F}_{\perp}} \\
=\|\left(\mathcal{N}_{\perp}+1\right)^{\frac{b}{2}} \mathbb{U}_{\mathcal{V}_{0}}^{*} \frac{\left(a^{\dagger}\left(\xi_{0}\right)\right)^{v_{0}}}{\sqrt{v_{0} !}} \cdots \frac{\left(a^{\dagger}\left(\xi_{k}\right)\right)^{v_{k}}}{\sqrt{v_{k} !}}|\Omega\rangle \|_{\mathcal{F}_{\perp}}^{2} \\
\quad \leq b^{b} C_{\mathcal{V}_{0}}^{b} \|\left(\mathcal{N}_{\perp}+1\right)^{\frac{b}{2}} \frac{\left(a^{\dagger}\left(\xi_{0}\right)\right)^{v_{0}}}{\sqrt{v_{0} !}} \cdots \frac{\left(a^{\dagger}\left(\xi_{k}\right)\right)^{v_{k}}}{\sqrt{v_{k} !}}|\Omega\rangle \|_{\mathcal{F}_{\perp}}^{2},
\end{gathered}
$$

where $C \mathcal{V}_{0}$ denotes the constant from Lemma 4.4 for $\mathcal{V}=\mathcal{V}_{0}$. This proves the estimate (4.26), because

$$
\begin{aligned}
& \left(\mathcal{N}_{\perp}+1\right)^{\frac{b}{2}} \frac{\left(a^{\dagger}\left(\xi_{0}\right)\right)^{v_{0}}}{\sqrt{v_{0} !}} \cdots \frac{\left(a^{\dagger}\left(\xi_{k}\right)\right)^{v_{k}}}{\sqrt{v_{k} !}}|\Omega\rangle \\
& \quad=\left(v_{0}+\cdots+v_{k}+1\right)^{\frac{b}{2}} \frac{\left(a^{\dagger}\left(\xi_{0}\right)\right)^{v_{0}}}{\sqrt{v_{0} !}} \cdots \frac{\left(a^{\dagger}\left(\xi_{k}\right)\right)^{v_{k}}}{\sqrt{v_{k} !}}|\Omega\rangle,
\end{aligned}
$$

and the bound (4.27) follows from the decomposition $\mathbb{P}_{0}^{(n)}=\sum_{m=1}^{\delta_{0}^{(n)}}\left|\chi_{0}^{(n, m)}\right\rangle\left\langle\chi_{0}^{(n, m)}\right|$.

Part (e). This follows from parts (a) and (c) and by Lemma 4.4, since

$$
\begin{aligned}
\left\langle\boldsymbol{\phi},\left(\mathbb{H}_{0}-E_{0}^{(0)}\right) \boldsymbol{\phi}\right\rangle_{\mathcal{F}_{\perp}} & =\left\langle\mathbb{U}_{\mathcal{V}_{0}} \phi, \sum_{j \geq 0} d^{(j)} a^{\dagger}\left(\xi_{j}\right) a\left(\xi_{j}\right) \mathbb{U}_{\mathcal{V}_{0}} \phi\right\rangle_{\mathcal{F}_{\perp}} \\
& \geq g_{0}^{(0)}\left\langle\phi, \mathbb{U}_{\mathcal{V}_{0}}^{*} \mathcal{N}_{\perp} \mathbb{U}_{\mathcal{V}_{0}} \phi\right\rangle_{\mathcal{F}_{\perp}} .
\end{aligned}
$$

Next, we recall that for excitation energies of order 1 , the eigenvalues of $\mathbb{H}_{\leq N}$ converge to eigenvalues of $\mathbb{H}_{0}$ as $N \rightarrow \infty$. Statements of this kind have been proven in $[65,27,36,44]$. 


\section{Lemma 4.8.}

(a) For any $v \in \mathbb{N}_{0}$ and $E^{(v)}$ as in Definition 2.3, there exists some $n \in \mathbb{N}_{0}$ such that

$$
\lim _{N \rightarrow \infty} E^{(v)}=E_{0}^{(n)}
$$

(b) In the sense of operators on $\mathcal{F}_{\perp}^{\leq N}$,

$$
\mathcal{N}_{\perp}+1 \leq \mathfrak{C}\left(\mathbb{H}_{\leq N}+N^{\frac{1}{3}}\right)
$$

(c) Let $\chi^{(n)} \in \mathfrak{E}^{(n)}$ for $n \in \mathbb{N}_{0}$. Then

$$
\left\langle\chi^{(n)},\left(\mathcal{N}_{\perp}+1\right) \chi^{(n)}\right\rangle_{\mathcal{F}_{\perp}} \leq \mathfrak{C}(n)
$$

and

$$
\left\langle\chi^{(n)},\left(\mathcal{N}_{\perp}+1\right)^{b} \chi^{(n)}\right\rangle_{\mathcal{F}_{\perp}} \leq \mathfrak{C}(b, n) N^{\frac{\ell}{3}}\left\langle\chi^{(n)},\left(\mathcal{N}_{\perp}+1\right)^{b-\ell} \chi^{(n)}\right\rangle_{\mathcal{F}_{\perp}}
$$

for $b \in \mathbb{N}_{0}$ and any $0 \leq \ell \leq b$. If $\varepsilon(N)=\mathcal{O}(1)$ in Assumption 3, one obtains the improved bound

$$
\left\langle\chi^{(n)},\left(\mathcal{N}_{\perp}+1\right)^{b} \chi^{(n)}\right\rangle_{\mathcal{F}_{\perp}} \leq\left(\mathfrak{C}(n)+3^{\frac{b}{2}}\right)^{b}
$$

Proof. Part (a). By Lemma 2.2 and Assumption 3, all assumptions (A1), (A2) and (A3s) in [36] are satisfied, hence part (a) follows from [36, Theorem 2.2(ii)].

Part (b). By Assumption 3, there exist constants $C_{1} \geq 0$ and $0<C_{2} \leq 1$ such that, for sufficiently large $N$,

$$
H_{N}-N e_{\mathrm{H}} \geq C_{2} \mathrm{~d} \Gamma_{\perp}(h)-C_{1} N^{\frac{1}{3}}
$$

in the sense of operators on $\mathfrak{S}^{N}$. Since $\varphi$ is the unique ground state of $h$ with eigenvalue 0 , it follows that

$$
\mathrm{d} \Gamma_{\perp}(h)=\sum_{j \geq 0} \varepsilon^{(j)} a^{\dagger}\left(\varphi_{j}\right) a\left(\varphi_{j}\right)=\sum_{j \geq 1} \varepsilon^{(j)} a^{\dagger}\left(\varphi_{j}\right) a\left(\varphi_{j}\right) \geq g_{\mathrm{H}} \mathcal{N}_{\perp}
$$

on $\mathfrak{H}^{N}$, where $0<\varepsilon^{(1)} \leq \varepsilon^{(2)} \leq \cdots$. Consequently, it holds for $\phi \in \mathcal{F}_{\perp}^{\leq N}$ that

$$
\begin{aligned}
\left\langle\boldsymbol{\phi}, \mathcal{N}_{\perp} \boldsymbol{\phi}\right\rangle_{\mathcal{F}_{\perp}^{\leq N}} & =\left\langle U_{N, \varphi}^{*} \boldsymbol{\phi}, \mathcal{N}_{\perp} U_{N, \varphi}^{*} \phi\right\rangle_{\mathfrak{H}^{N}} \\
& \leq \frac{1}{C_{2} g_{\mathrm{H}}}\left\langle\boldsymbol{\phi},\left(\mathbb{H}_{\leq N}+C_{1} N^{\frac{1}{3}}\right) \boldsymbol{\phi}\right\rangle_{\mathcal{F}_{\perp}^{\leq N}} .
\end{aligned}
$$

Part (c). By Lemma 2.2 and Assumption 3, [36, Theorem 2.2(iv)] implies that there exists some $\chi_{0}^{(n)} \in \mathfrak{E}_{0}^{(n)}$ such that, up to a subsequence,

$$
\lim _{N \rightarrow \infty}\left\|\chi^{(n)}-\chi_{0}^{(n)}\right\|_{\mathcal{F}_{\perp}}=0, \quad \lim _{N \rightarrow \infty}\left\langle\left(\chi^{(n)}-\chi_{0}^{(n)}\right), \mathbb{H}_{0}\left(\chi^{(n)}-\chi_{0}^{(n)}\right)\right\rangle_{\mathcal{F}_{\perp}}=0,
$$


hence, by Lemma 4.7e,

$$
\begin{aligned}
\left\langle\chi^{(n)},\left(\mathcal{N}_{\perp}+1\right) \chi^{(n)}\right\rangle_{\mathcal{F}_{\perp}} \\
\leq \mathbb{C}\left\langle\left(\chi^{(n)}-\chi_{0}^{(n)}\right),\left(\mathbb{H}_{0}-E_{0}^{(0)}+1\right)\left(\chi^{(n)}-\chi_{0}^{(n)}\right)\right\rangle_{\mathcal{F}_{\perp}} \\
\quad+\mathfrak{C}\left\langle\chi_{0}^{(n)},\left(\mathbb{H}_{0}-E_{0}^{(0)}+1\right) \chi_{0}^{(n)}\right\rangle_{\mathcal{F}_{\perp}} \\
\quad+2 \mathfrak{C}\left\|\chi^{(n)}-\chi_{0}^{(n)}\right\|_{\mathcal{F}_{\perp}}\left\|\left(\mathbb{H}_{0}-E_{0}^{(0)}+1\right) \chi_{0}^{(n)}\right\|_{\mathcal{F}_{\perp}} \\
\leq \mathbb{C}\left(E_{0}^{(n)}-E_{0}^{(0)}+1\right)
\end{aligned}
$$

for sufficiently large $N$. Further, part (b) implies that

$$
\begin{aligned}
\left\langle\chi^{(n)},\left(\mathcal{N}_{\perp}+1\right)^{b+1} \chi^{(n)}\right\rangle_{\mathcal{F}_{\perp}} \\
=\left\langle\left(\mathcal{N}_{\perp}+1\right)^{\frac{b}{2}} \chi^{(n)},\left(\mathcal{N}_{\perp}+1\right)\left(\mathcal{N}_{\perp}+1\right)^{\frac{b}{2}} \chi^{(n)}\right\rangle_{\mathcal{F}_{\perp}^{\leq N}} \\
\leq \mathbb{C}\left\langle\left(\mathcal{N}_{\perp}+1\right)^{\frac{b}{2}} \chi^{(n)},\left(\mathbb{H}_{\leq N}+N^{\frac{1}{3}}\right)\left(\mathcal{N}_{\perp}+1\right)^{\frac{b}{2}} \chi^{(n)}\right\rangle_{\mathcal{F}_{\perp}^{\leq N}} \\
\leq \mathbb{C}\left\langle\left(\mathcal{N}_{\perp}+1\right)^{b} \chi^{(n)},\left(\mathbb{H}_{\leq N}+N^{\frac{1}{3}}\right) \chi_{\leq N}^{(n)}\right\rangle_{\mathcal{F}_{\perp}^{\leq N}} \\
\quad+\mathbb{C}\left\|\left(\mathcal{N}_{\perp}+1\right)^{\frac{b}{2}} \chi^{(n)}\right\|_{\mathcal{F}_{\perp}^{\leq N}}\left\|\left[\mathbb{H}_{\leq N},\left(\mathcal{N}_{\perp}+1\right)^{\frac{b}{2}}\right] \chi^{(n)}\right\|_{\mathcal{F}_{\perp}^{\leq N}} \\
\leq \mathbb{C}\left(\left|E_{0}^{(n)}\right|+N^{\frac{1}{3}}+3^{\frac{b}{2}}\right)\left\langle\chi^{(n)},\left(\mathcal{N}_{\perp}+1\right)^{b} \chi^{(n)}\right\rangle_{\mathcal{F}_{\perp}}
\end{aligned}
$$

by Lemma $5.2 \mathrm{~b}$ and since $\chi^{(n)} \in \mathfrak{E}^{(n)}$. Iterating over $b$ concludes the proof.

\section{Proofs}

In the remainder of the paper, we abbreviate

$$
\|\cdot\|_{\mathcal{F}_{\perp}} \equiv\|\cdot\|, \quad\langle\cdot, \cdot\rangle_{\mathcal{F}_{\perp}} \equiv\langle\cdot, \cdot\rangle, \quad\|\cdot\|_{\mathcal{L}\left(\mathcal{F}_{\perp}\right)} \equiv\|\cdot\|_{\text {op }}, \quad \operatorname{Tr}_{\mathcal{F}_{\perp}} \equiv \operatorname{Tr}
$$

We will always assume that Assumptions 1,2 and 3 are satisfied.

\subsection{Asymptotic expansion of $\mathbb{P}^{(n)}$}

\subsubsection{Proof of Lemma 3.13}

Recall that $\mathbb{H}=\mathbb{H}^{<}+\mathbb{H}^{>}$, by equation (3.39), hence

$$
\frac{1}{z-\mathbb{H}}=\frac{1}{z-\mathbb{H}^{<}}\left(z-\mathbb{H}+\mathbb{H}^{>}\right) \frac{1}{z-\mathbb{H}}=\frac{1}{z-\mathbb{H}^{<}}+\frac{1}{z-\mathbb{H}^{<}} \mathbb{H}^{>} \frac{1}{z-\mathbb{H}} .
$$

Next, we prove by induction over $a \in \mathbb{N}_{0}$ that

$$
\frac{1}{z-\mathbb{H}^{<}}=\frac{1}{z-\mathbb{H}_{0}} \sum_{\ell=0}^{a} \lambda_{N}^{\frac{\ell}{2}} \mathbb{T}_{\ell}(z)+\lambda_{N}^{\frac{a+1}{2}} \frac{1}{z-\mathbb{H}^{<}} \sum_{\nu=0}^{a} \mathbb{R}_{v} \frac{1}{z-\mathbb{H}_{0}} \mathbb{T}_{a-v}(z),
$$


where

$$
\mathbb{T}_{\ell}(z)=\sum_{v=1}^{\ell} \mathbb{H}_{v} \frac{1}{z-\mathbb{H}_{0}} \mathbb{T}_{a-v}(z), \quad \mathbb{T}_{0}(z)=\mathbb{1}
$$

Base case. Proposition 3.12 implies that

$$
\mathbb{H}^{<}=\mathbb{H}_{0}+\lambda_{N}^{\frac{1}{2}} \mathbb{R}_{0}
$$

hence

$$
\begin{aligned}
\frac{1}{z-\mathbb{H}^{<}} & =\frac{1}{z-\mathbb{H}^{<}}\left(z-\mathbb{H}^{<}+\lambda_{N}^{\frac{1}{2}} \mathbb{R}_{0}\right) \frac{1}{z-\mathbb{H}_{0}} \\
& =\frac{1}{z-\mathbb{H}_{0}}+\lambda_{N}^{\frac{1}{2}} \frac{1}{z-\mathbb{H}^{<}} \mathbb{R}_{0} \frac{1}{z-\mathbb{H}_{0}} .
\end{aligned}
$$

Induction step. Assume equation (5.2) holds for $a-1 \in \mathbb{N}$. Since

$$
\mathbb{H}^{<}=\sum_{j=0}^{v} \lambda_{N}^{\frac{j}{2}} \mathbb{H}_{j}+\lambda_{N}^{\frac{v+1}{2}} \mathbb{R}_{\nu}=\sum_{j=0}^{v} \lambda_{N}^{\frac{j}{2}} \mathbb{H}_{j}+\lambda_{N}^{\frac{v+1}{2}} \mathbb{H}_{\nu+1}+\lambda_{N}^{\frac{v+2}{2}} \mathbb{R}_{\nu+1},
$$

it follows that

$$
\mathbb{R}_{v}=\mathbb{H}_{v+1}+\lambda_{N}^{\frac{1}{2}} \mathbb{R}_{v+1}
$$

hence we conclude with equation (5.5) and by the induction hypothesis that

$$
\begin{aligned}
\frac{1}{z-\mathbb{H}^{<}}= & \frac{1}{z-\mathbb{H}_{0}} \sum_{\ell=0}^{a-1} \lambda_{N}^{\frac{\ell}{2}} \mathbb{T}_{\ell}(z) \\
& +\lambda_{N}^{\frac{a}{2}} \frac{1}{z-\mathbb{H}^{<}} \sum_{\nu=0}^{a-1}\left(\mathbb{H}_{v+1}+\lambda_{N}^{\frac{1}{2}} \mathbb{R}_{v+1}\right) \frac{1}{z-\mathbb{H}_{0}} \mathbb{T}_{a-v-1}(z) \\
= & \frac{1}{z-\mathbb{H}_{0}} \sum_{\ell=0}^{a-1} \lambda_{N}^{\frac{\ell}{2}} \mathbb{T}_{\ell}(z)+\lambda_{N}^{\frac{a}{2}} \frac{1}{z-\mathbb{H}_{0}} \sum_{v=0}^{a-1} \mathbb{H}_{v+1} \frac{1}{z-\mathbb{H}_{0}} \mathbb{T}_{a-v-1}(z) \\
& +\lambda_{N}^{\frac{a+1}{2}} \frac{1}{z-\mathbb{H}^{<}} \mathbb{R}_{0} \frac{1}{z-\mathbb{H}_{0}} \sum_{v=0}^{a-1} \mathbb{H}_{v+1} \frac{1}{z-\mathbb{H}_{0}} \mathbb{T}_{a-v-1}(z) \\
& +\lambda_{N}^{\frac{a+1}{2}} \frac{1}{z-\mathbb{H}^{<}} \sum_{v=0}^{a-1} \mathbb{R}_{v+1} \frac{1}{z-\mathbb{H}_{0}} \mathbb{T}_{a-v-1}(z) \\
= & \frac{1}{z-\mathbb{H}_{0}} \sum_{\ell=0}^{a} \lambda_{N}^{\frac{\ell}{2}} \mathbb{T}_{\ell}(z) \\
& +\lambda_{N}^{\frac{a+1}{2}} \frac{1}{z-\mathbb{H}^{<}}\left(\mathbb{R}_{0} \frac{1}{z-\mathbb{H}_{0}} \mathbb{T}_{a}+\sum_{\nu=0}^{a-1} \mathbb{R}_{v+1} \frac{1}{z-\mathbb{H}_{0}} \mathbb{T}_{a-v-1}(z)\right) \\
= & \frac{1}{z-\mathbb{H}_{0}} \sum_{\ell=0}^{a} \lambda_{N}^{\frac{\ell}{2}} \mathbb{T}_{\ell}(z)+\lambda_{N}^{\frac{a+1}{2}} \frac{1}{z-\mathbb{H}^{<}} \sum_{v=0}^{a} \mathbb{R}_{v} \frac{1}{z-\mathbb{H}_{0}} \mathbb{T}_{a-v}(z),
\end{aligned}
$$


which concludes the induction. Finally, we rewrite $\mathbb{T}_{a}(z)$ as

$$
\begin{aligned}
& \mathbb{T}_{a}(z)=\sum_{j_{1}=1}^{a} \mathbb{H}_{j_{1}} \frac{1}{z-\mathbb{H}_{0}} \mathbb{T}_{a-j_{1}}(z) \\
& =\sum_{j_{1}=1}^{a} \sum_{j_{2}=1}^{a-j_{1}} \mathbb{H}_{j_{1}} \frac{1}{z-\mathbb{H}_{0}} \mathbb{H}_{j_{2}} \frac{1}{z-\mathbb{H}_{0}} \mathbb{T}_{a-\left(j_{1}+j_{2}\right)}(z) \\
& =\sum_{v=1}^{a} \sum_{\substack{\boldsymbol{j} \in \mathbb{N}^{v} \\
|\boldsymbol{j}|=a}} \mathbb{H}_{j_{1}} \frac{1}{z-\mathbb{H}_{0}} \cdots \mathbb{H}_{j_{v}} \frac{1}{z-\mathbb{H}_{0}} \mathbb{T}_{0}(z),
\end{aligned}
$$

which concludes the proof.

\subsubsection{Proof of Proposition 3.14}

Let $n \in \mathbb{N}_{0}$. The expansion of the resolvent from Lemma 3.13 yields

$$
\mathbb{P}^{(n)}=\mathbb{P}_{0}^{(n)}+\sum_{\ell=1}^{a} \lambda_{N}^{\frac{\ell}{2}} \sum_{\nu=1}^{\ell} \sum_{\substack{\boldsymbol{j} \in \mathbb{N}^{v} \\|\boldsymbol{j}|=\ell}} \mathbb{A}_{\boldsymbol{j}}^{(n)}+\lambda_{N}^{\frac{a+1}{2}} \sum_{\nu=0}^{a} \sum_{m=1}^{a-v} \sum_{\substack{\boldsymbol{j} \in \mathbb{N}^{m} \\|\boldsymbol{j}|=a-\nu}} \mathbb{B}_{j}^{(n)}+\mathbb{C}^{(n)},
$$

where

$$
\begin{aligned}
\mathbb{A}_{j}^{(n)} & :=\frac{1}{2 \pi \mathrm{i}} \oint_{\gamma^{(n)}} \frac{1}{z-\mathbb{H}_{0}} \mathbb{H}_{j_{1}} \frac{1}{z-\mathbb{H}_{0}} \mathbb{H}_{j_{2}} \frac{1}{z-\mathbb{H}_{0}} \cdots \mathbb{H}_{j_{v}} \frac{1}{z-\mathbb{H}_{0}} \mathrm{~d} z, \\
\mathbb{B}_{j}^{(n)} & :=\frac{1}{2 \pi \mathrm{i}} \oint_{\gamma^{(n)}} \frac{1}{z-\mathbb{H}^{<}} \mathbb{R}_{v} \frac{1}{z-\mathbb{H}_{0}} \mathbb{H}_{j_{1}} \frac{1}{z-\mathbb{H}_{0}} \cdots \mathbb{H}_{j_{m}} \frac{1}{z-\mathbb{H}_{0}} \mathrm{~d} z, \\
\mathbb{C}^{(n)} & :=\frac{1}{2 \pi \mathrm{i}} \oint_{\gamma^{(n)}} \frac{1}{z-\mathbb{H}^{<}} \mathbb{H}^{>} \frac{1}{z-\mathbb{H}} \mathrm{d} z .
\end{aligned}
$$

Computation of $\mathbb{A}_{j}^{(n)}$. We decompose $1=\mathbb{P}_{0}^{(n)}+\mathbb{Q}_{0}^{(n)}$ in each term in definition (5.11) and sort according to the number of projections $\mathbb{Q}_{0}^{(n)}$, which takes the values $k=0, \ldots, v+1$. This yields

$$
\begin{aligned}
\mathbb{A}_{j}^{(n)} & =\sum_{k=0}^{v+1} \sum_{\substack{\boldsymbol{m} \in\{0,1\}^{\gamma+1} \\
|\boldsymbol{m}|=k}} \frac{1}{2 \pi \mathrm{i}} \oint_{\gamma^{(n)}} \frac{1}{\left(z-E_{0}^{(n)}\right)^{v+1-k}} \widetilde{\mathbb{O}}_{m_{1}}^{(n)}(z) \mathbb{H}_{j_{1}} \cdots \widetilde{\mathbb{O}}_{m_{\nu}}^{(n)}(z) \mathbb{H}_{j_{v}} \widetilde{\mathbb{O}}_{m_{\nu+1}}^{(n)}(z) \mathrm{d} z \\
& =: \sum_{k=0}^{v+1} \widetilde{\mathbb{A}}_{k, \boldsymbol{j}}^{(n)},
\end{aligned}
$$

where we abbreviate

$$
\widetilde{\mathbb{O}}_{0}^{(n)}(z):=\mathbb{P}_{0}^{(n)}, \quad \widetilde{\mathbb{O}}_{1}^{(n)}(z):=\frac{\mathbb{Q}_{0}^{(n)}}{z-\mathbb{H}_{0}}
$$

Observe first that the contributions with exclusively $\mathbb{P}_{0}^{(n)}(k=v+1)$ or exclusively $\mathbb{Q}_{0}^{(n)}(k=0)$ vanish: 
in case of only $\mathbb{P}_{0}^{(n)}$,

$$
\widetilde{\mathbb{A}}_{0, \boldsymbol{j}}^{(n)}=\frac{1}{2 \pi \mathrm{i}}\left(\oint_{\gamma^{(n)}} \frac{1}{\left(z-E_{0}^{(n)}\right)^{\nu+1}} \mathrm{~d} z\right) \mathbb{P}_{0}^{(n)} \mathbb{H}_{j_{1}} \mathbb{P}_{0}^{(n)} \cdots \mathbb{P}_{0}^{(n)} \mathbb{H}_{j_{v}} \mathbb{P}_{0}^{(n)}=0,
$$

and in case of only $\mathbb{Q}_{0}^{(n)}$, the integrand is holomorphic in the area enclosed by $\gamma^{(n)}$, hence $\widetilde{\mathbb{A}}_{v+1, j}^{(n)}=0$.

For $1 \leq k \leq v$, the integrand in $\widetilde{\mathbb{A}}_{k, j}^{(n)}$ has a pole of order $v+1-k$ at $z=E_{0}^{(n)}$, hence the residue theorem implies that

$$
\widetilde{\mathbb{A}}_{k, j}^{(n)}=\sum_{\substack{\boldsymbol{m} \in\{0,1\}^{v+1} \\|\boldsymbol{m}|=k}} \frac{1}{(v-k) !} \lim _{z \rightarrow E_{0}^{(n)}} \frac{\mathrm{d}^{\nu-k}}{\mathrm{~d} z^{\nu-k}}\left(\widetilde{\mathbb{O}}_{m_{1}}^{(n)}(z) \mathbb{H}_{j_{1}} \cdots \widetilde{\mathbb{O}}_{m_{\nu}}^{(n)}(z) \mathbb{H}_{j_{v}} \widetilde{\mathbb{O}}_{m_{\nu+1}}^{(n)}(z)\right)
$$

Let us consider the case where $m_{j}=1$ for $j=1, \ldots, k$ and $m_{j}=0$ for $j=k+1, \ldots, v+1$. By the Leibniz rule and since

$$
\left.\frac{\mathrm{d}^{m}}{\mathrm{~d} z^{m}} \widetilde{\mathbb{O}}_{1}^{(n)}(z)\right|_{z=E_{0}^{(n)}}=(-1)^{m} m ! \mathbb{O}_{m+1}^{(n)}
$$

with $\mathbb{O}_{k}^{(n)}$ and $\mathbb{O}_{0}^{(n)}$ as in definition (3.8), that is,

$$
\mathbb{O}_{0}^{(n)}=-\mathbb{P}_{0}^{(n)}, \quad \mathbb{O}_{k}^{(n)}=\frac{\mathbb{Q}_{0}^{(n)}}{\left(E_{0}^{(n)}-\mathbb{H}_{0}\right)^{k}}
$$

we obtain for this case

$$
\begin{aligned}
& \frac{1}{(v-k) !} \lim _{z \rightarrow E_{0}^{(n)}} \frac{\mathrm{d}^{\nu-k}}{\mathrm{~d} z^{v-k}}\left(\widetilde{\mathbb{O}}_{1}^{(n)}(z) \mathbb{H}_{j_{1}} \cdots \widetilde{\mathbb{O}}_{1}^{(n)}(z)\right) \mathbb{H}_{j_{k}} \mathbb{P}_{0}^{(n)} \mathbb{H}_{j_{k+1}} \cdots \mathbb{P}_{0}^{(n)} \mathbb{H}_{j_{v}} \mathbb{P}_{0}^{(n)} \\
& =\frac{1}{(v-k) !} \sum_{\substack{\boldsymbol{m} \in \mathbb{N}_{0}^{k} \\
|\boldsymbol{m}|=v-k}}\left(\begin{array}{c}
v-k \\
\boldsymbol{m}
\end{array}\right)\left(\left.\frac{\mathrm{d}^{m_{1}}}{\mathrm{~d} z^{m_{1}}} \widetilde{\mathbb{O}}_{1}^{(n)}(z)\right|_{z=E_{0}^{(n)}}\right) \mathbb{H}_{j_{1}} \cdots \\
& \quad \ldots\left(\left.\frac{\mathrm{d}^{m_{k}}}{\mathrm{~d} z^{m_{k}}} \widetilde{\mathbb{O}}_{1}^{(n)}(z)\right|_{z=E_{0}^{(n)}}\right) \mathbb{H}_{j_{k}} \mathbb{P}_{0}^{(n)} \mathbb{H}_{j_{k+1}} \cdots \mathbb{P}_{0}^{(n)} \mathbb{H}_{j_{v}} \mathbb{P}_{0}^{(n)} \\
& =-\sum_{\substack{\boldsymbol{m} \in \mathbb{N}_{0}^{k} \\
|\boldsymbol{m}|=v-k}} \mathbb{O}_{m_{1}+1}^{(n)} \mathbb{H}_{j_{1}} \cdots \mathbb{O}_{m_{k}+1}^{(n)} \mathbb{H}_{j_{k}} \mathbb{O}_{0}^{(n)} \mathbb{H}_{j_{k+1}} \cdots \mathbb{O}_{0}^{(n)} \mathbb{H}_{j_{v}} \mathbb{O}_{0}^{(n)} \\
& =-\sum_{\substack{\boldsymbol{m} \in \mathbb{N}^{k} \\
|\boldsymbol{m}|=v}} \mathbb{O}_{m_{1}}^{(n)} \mathbb{H}_{j_{1}} \cdots \mathbb{O}_{m_{k}}^{(n)} \mathbb{H}_{j_{k}} \mathbb{O}_{0}^{(n)} \mathbb{H}_{j_{k+1}} \cdots \mathbb{O}_{0}^{(n)} \mathbb{H}_{j_{v}} \mathbb{O}_{0}^{(n)} . \\
&
\end{aligned}
$$

The other contributions to $\widetilde{\mathbb{A}}_{k, j}^{(n)}$ are related to equation (5.20) through permutations, hence

$$
\widetilde{\mathbb{A}}_{k, j}^{(n)}=-\sum_{\substack{\boldsymbol{m} \in \mathbb{N}^{k} \times\{0\}^{\nu-k+1} \\ \text { nline by Cambridge Upiryersity Press }}} \mathbb{O}_{m_{1}}^{(n)} \mathbb{H} \mathbb{H}_{j_{1}} \cdots \mathbb{O}_{m_{\nu}}^{(n)} \mathbb{H} \mathbb{H}_{j_{\nu}} \mathbb{O}_{m_{\nu+1}}^{(n)}
$$


and consequently

$$
\mathbb{A}_{j}^{(n)}=\sum_{k=1}^{v} \widetilde{\mathbb{A}}_{k, \boldsymbol{j}}^{(n)}=-\sum_{\substack{\boldsymbol{m} \in \mathbb{N}_{0}^{v+1} \\|\boldsymbol{m}|=\nu}} \mathbb{O}_{m_{1}}^{(n)} \mathbb{H}_{j_{1}} \cdots \mathbb{O}_{m_{v}}^{(n)} \mathbb{H}_{j_{v}} \mathbb{O}_{m_{v+1}}^{(n)}
$$

Computation of $\mathbb{B}_{j}^{(n)}$. Decomposing the first identity in definition $(5.12)$ as $1=\mathbb{P}^{(n)}+\mathbb{Q}^{(n)}$, we note that the term with $\mathbb{P}^{(n)}$ yields $\mathbb{B}_{P}^{(n)}$. For the term with $\mathbb{Q}^{(n)}$, we decompose in each resolvent of $\mathbb{H}_{0}$ the identity as $1=\mathbb{P}_{0}^{(n)}+\mathbb{Q}_{0}^{(n)}$. Note that the term containing exclusively $\mathbb{Q}^{(n)}$ and $\mathbb{Q}_{0}^{(n)}$ vanishes, since the integrand has no poles in the area enclosed by $\gamma^{(n)}$.

Computation of $\mathbb{C}^{(n)}$. Recall that $\mathbb{P}^{(n)}$ projects onto a subspace of $\mathcal{F}_{\perp}^{\leq N} \oplus 0$, hence

$$
\mathbb{P}^{(n)} \mathbb{H}^{>}=\mathbb{H}^{>} \mathbb{P}^{(n)}=0 .
$$

Consequently, decomposing both identities in $\mathbb{C}^{(n)}$ yields

$$
\mathbb{C}^{(n)}=\frac{1}{2 \pi \mathrm{i}} \oint_{\gamma^{(n)}} \frac{\mathbb{Q}^{(n)}}{z-\mathbb{H}^{<}} \mathbb{H}^{>} \frac{\mathbb{Q}^{(n)}}{z-\mathbb{H}} \mathrm{d} z=0,
$$

since the integrand is holomorphic in the area enclosed by $\gamma^{(n)}$.

\subsection{Auxiliary estimates}

\subsubsection{Preliminaries}

In this section, we collect some preliminary estimates. First, we provide bounds for second-quantised $m$-body operators; subsequently, we estimate $\mathbb{K}_{j}, \mathbb{H}_{j}$ and $\mathbb{R}_{j}$ as well as commutators of $\mathcal{N}_{\perp}$ with $\mathbb{H}_{\leq N}$ and $\mathbb{H}$.

Lemma 5.1. Let $m \in \mathbb{N}$ and let $O^{(m)}$ be an operator on $\mathfrak{H}^{m}$. Assume that there exist constants $c_{1}, c_{2} \geq 0$ such that

$$
\left\|O^{(m)} \psi\right\|_{\mathfrak{S}^{m}}^{2} \leq c_{1}\left\|\sum_{j=1}^{m} T_{j} \psi\right\|_{\mathfrak{Y}^{m}}^{2}+c_{2}\|\psi\|_{\mathfrak{S}^{m}}^{2}
$$

for any $\psi \in \mathcal{D}\left(\sum_{j=1}^{m} T_{j}\right)$ and with $T$ as in definition (2.2).

(a) Let $\psi \in \mathfrak{S}^{m}$. Then

$$
\left\|O^{(m)} \psi\right\|_{\mathfrak{S}^{m}}^{2} \leq 2 c_{1}\left\|\sum_{j=1}^{m} h_{j} \psi\right\|_{\mathfrak{S}^{m}}^{2}+2 c_{3}\|\psi\|_{\mathfrak{W}^{m}}^{2},
$$

where $c_{3}=\mathfrak{C}_{c_{1}} m^{2}+\frac{c_{2}}{2}$.

(b) Let $k \geq m$ and $\psi \in \mathfrak{S}_{\text {sym. }}^{k}$. Then

$$
\left\|\sum_{j=1}^{m} h_{j} \psi\right\|_{\mathfrak{S}^{k}}^{2} \leq \frac{m}{k}\left\|\sum_{j=1}^{k} h_{j} \psi\right\|_{\mathfrak{S}^{k}}^{2}
$$


(c) Let $k \geq m$. Then it follows for $\psi_{k} \in \mathfrak{S}_{\text {sym }}^{k}$ that

$$
\left\|\sum_{1 \leq j_{1}<\cdots<j_{m} \leq k} O_{j_{1}, \ldots, j_{m}}^{(m)} \psi_{k}\right\|_{\mathfrak{G}^{k}}^{2} \leq\left(\begin{array}{c}
k \\
m
\end{array}\right)^{2}\left(\frac{2 c_{1} m}{k}\left\|\sum_{j=1}^{k} h_{j} \psi_{k}\right\|_{\mathfrak{G}^{k}}^{2}+2 c_{3}\left\|\psi_{k}\right\|_{\mathfrak{S}^{k}}^{2}\right) .
$$

Proof. Part (a) follows since $h_{j}=T_{j}+\left(v * \varphi^{2}\right)\left(x_{j}\right)-\mu_{\mathrm{H}}$ and by inequality (2.5), because

$$
\begin{aligned}
\left\|O^{(m)} \psi\right\|_{\mathfrak{H}^{m}}^{2} & \leq c_{1}\left(\left\|\sum_{j=1}^{m} h_{j} \psi\right\|_{\mathfrak{T}^{m}}+\left\|\sum_{j=1}^{m}\left(v * \varphi^{2}\left(x_{j}\right)-\mu_{\mathrm{H}}\right) \psi\right\|_{\mathfrak{H}^{m}}\right)^{2}+c_{2}\|\psi\|_{\mathfrak{H}^{m}}^{2} \\
& \leq 2 c_{1}\left\|\sum_{j=1}^{m} h_{j} \psi\right\|^{2}+\left(2 c_{1} \mathfrak{C} m^{2}+c_{2}\right)\|\psi\|_{\mathfrak{H}^{m}}^{2} .
\end{aligned}
$$

For part (b), the permutation symmetry of $\psi$ leads to the estimate

$$
\begin{aligned}
\left\|\sum_{j=1}^{m} h_{j} \psi\right\|_{\mathfrak{H}^{k}}^{2} & =m\left\langle\psi, h_{1} h_{1} \psi\right\rangle_{\mathfrak{S}^{k}}+m(m-1)\left\langle\psi, h_{1} h_{2} \psi\right\rangle_{\mathfrak{H}^{k}} \\
& =\frac{m}{k} \sum_{j=1}^{k}\left\langle\psi, h_{j} h_{j} \psi\right\rangle_{\mathfrak{S}^{k}}+\frac{m(m-1)}{k(k-1)} \sum_{\substack{1 \leq j, \ell \leq k \\
\neq j}}\left\langle\psi, h_{j} h_{\ell} \psi\right\rangle_{\mathfrak{H}^{k}} \\
& \leq \frac{m}{k} \sum_{1 \leq j, \ell \leq k}\left\langle\psi, h_{j} h_{\ell} \psi\right\rangle_{\mathfrak{H}^{k}},
\end{aligned}
$$

since $m \leq k$ and $h \geq 0$. For part (c), we obtain with parts (a) and (b)

$$
\left\|O_{1, \ldots, m}^{(m)} \psi_{k}\right\|_{\mathfrak{S}^{k}}^{2} \leq \frac{2 c_{1} m}{k}\left\|\sum_{j=1}^{k} h_{j} \psi_{k}\right\|_{\mathfrak{G}^{k}}^{2}+2 c_{3}\left\|\psi_{k}\right\|_{\mathfrak{H}^{k}}^{2}
$$

which proves the claim since

$$
\left\|\sum_{1 \leq j_{1}<\cdots<j_{m} \leq k} O_{j_{1}, \ldots, j_{m}}^{(m)} \psi_{k}\right\|_{\mathfrak{g}^{k}}^{2} \leq\left(\sum_{1 \leq j_{1}<\cdots<j_{m} \leq k}\left\|O_{j_{1}, \ldots, j_{m}}^{(m)} \psi_{k}\right\|\right)^{2} .
$$

In the next lemma, we collect bounds for the operators $\mathbb{K}_{1}$ to $\mathbb{K}_{4}$ from definition (2.41):

Lemma 5.2. Let $\phi \in \mathcal{F}_{\perp}$. 
(a) $\operatorname{For} \mathbb{K}_{j}^{(*)} \in\left\{\mathbb{K}_{j}, \mathbb{K}_{j}^{*}\right\}$,

$$
\begin{aligned}
\left\|\mathbb{K}_{1} \phi\right\| & \leq \mathfrak{C}\left\|\left(\mathcal{N}_{\perp}+1\right) \phi\right\|, \\
\left\|\mathbb{K}_{2}^{(*)} \phi\right\| & \leq \mathfrak{C}\left\|\left(\mathcal{N}_{\perp}+1\right) \phi\right\|, \\
\left\|\mathbb{K}_{3}^{(*)} \phi\right\| & \leq \mathfrak{C}\left\|\left(\mathcal{N}_{\perp}+1\right)^{\frac{3}{2}} \boldsymbol{\phi}\right\|, \\
\left\|\mathbb{K}_{4} \phi\right\| & \leq \mathfrak{C}\left(\left\|\left(\mathcal{N}_{\perp}+1\right)^{2} \phi\right\|+\left\|\mathbb{K}_{0}^{\frac{1}{2}}\left(\mathcal{N}_{\perp}+1\right)^{\frac{3}{2}} \boldsymbol{\phi}\right\|\right) \\
& \leq \mathfrak{C}\left(\left\|\left(\mathcal{N}_{\perp}+1\right)^{2} \boldsymbol{\phi}\right\|+\left\|\mathbb{H}_{0}\left(\mathcal{N}_{\perp}+1\right)^{\frac{3}{2}} \boldsymbol{\phi}\right\|\right) .
\end{aligned}
$$

(b) Let $\ell \geq 0$. Then

$$
\begin{aligned}
\left\|\left[\mathbb{H}_{\leq N},\left(\mathcal{N}_{\perp}+1\right)^{\ell}\right] \boldsymbol{\phi}\right\|_{\mathcal{F}_{\perp} \leq N} & \leq 3^{\ell} \mathfrak{C}\left\|\left(\mathcal{N}_{\perp}+1\right)^{\ell} \phi\right\|_{\mathcal{F}_{\perp} \leq N} \\
\left\|\left[\mathbb{H}_{0},\left(\mathcal{N}_{\perp}+1\right)^{\ell}\right] \boldsymbol{\phi}\right\| & \leq 3^{\ell} \ell \mathfrak{C}\left\|\left(\mathcal{N}_{\perp}+1\right)^{\ell} \boldsymbol{\phi}\right\|
\end{aligned}
$$

Proof. Part (a). Since $\|K\|_{\mathfrak{H} \rightarrow \mathfrak{H}} \leq\|K\|_{\mathrm{HS}} \leq \mathfrak{C}$ by the estimate (2.6), and as inequalities (2.3) and (2.5) imply that

$$
\left\|K_{3} \psi\right\|_{\mathfrak{W}_{\perp}^{2}} \leq\left\|v\left(x_{1}-x_{2}\right) \varphi\left(x_{1}\right) \psi\left(x_{2}\right)\right\|_{\mathfrak{G}_{\perp}^{2}}+\left\|\left(v * \varphi^{2}\right)\left(x_{1}\right) \varphi\left(x_{1}\right) \psi\left(x_{2}\right)\right\|_{\mathfrak{G}_{\perp}^{2}} \leq \mathbb{C}\|\psi\|
$$

for any $\psi \in \mathfrak{H}_{\perp}$, the bounds for $\mathbb{K}_{1}, \mathbb{K}_{2}^{(*)}$ and $\mathbb{K}_{3}^{(*)}$ follow from Lemma 4.1. Finally, note that

$$
\mathbb{K}_{4}=\mathrm{d} \Gamma_{\perp}(v)-\mathrm{d} \Gamma_{\perp}\left(\widetilde{K_{4}}\right)
$$

where $\widetilde{K_{4}}$ denotes the multiplication operator on $\mathfrak{H}_{\perp} \otimes \mathfrak{H}_{\perp}$ corresponding to

$$
\widetilde{K_{4}}\left(x_{1}, x_{2}\right):=\left(v * \varphi^{2}\right)\left(x_{1}\right)+\left(v * \varphi^{2}\right)\left(x_{2}\right)-\left\langle\varphi, v * \varphi^{2} \varphi\right\rangle .
$$

As before, the bound (2.5) and Lemma 4.1 imply that $\left\|\mathrm{d} \Gamma_{\perp}\left(\widetilde{K_{4}}\right) \boldsymbol{\phi}\right\| \leq \mathbb{C}\left\|\left(\mathcal{N}_{\perp}+1\right)^{2} \phi\right\|$. Moreover,

$$
\begin{aligned}
\left\langle\psi,\left|v\left(x_{1}-x_{2}\right)\right|^{2} \psi\right\rangle_{\mathfrak{S}^{k}} & \leq \mathbb{C}\left(\|\psi\|_{\mathfrak{S}^{k}}^{2}+\left\langle\psi, h_{1} \psi\right\rangle_{\mathfrak{G}^{k}}+\left\|v * \varphi^{2}-\mu_{\mathrm{H}}\right\|_{\infty}\|\psi\|_{\mathfrak{S}^{k}}^{2}\right) \\
& \leq \mathbb{C}\left(\|\psi\|_{\mathfrak{S}^{k}}^{2}+\frac{1}{k}\left\langle\psi, \sum_{j=1}^{k} h_{j} \psi\right\rangle_{\mathfrak{Y}^{k}}\right)
\end{aligned}
$$

for $\psi \in \mathfrak{H}^{k}$ by inequalities (2.4) and (2.5); hence it follows from Lemmas 4.1 and 5.1c that

$$
\begin{aligned}
\left\|\mathrm{d} \Gamma_{\perp}(v) \boldsymbol{\phi}\right\|^{2} & \leq \sum_{k \geq 0}\left\|\sum_{1 \leq i<j \leq k} v\left(x_{i}-x_{j}\right) \phi^{(k)}\right\|_{\mathfrak{H}_{\perp}^{k}}^{2} \\
& \leq \mathbb{C}\left(\sum_{k \geq 0} k(k-1)^{2}\left\langle\phi^{(k)}, \mathbb{K}_{0} \phi^{(k)}\right\rangle_{\mathfrak{S}^{k}}+\left\|\left(\mathcal{N}_{\perp}+1\right)^{2} \boldsymbol{\phi}\right\|^{2}\right) \\
& \leq \mathbb{C}\left(\left\|\mathbb{K}_{0}^{\frac{1}{2}}\left(\mathcal{N}_{\perp}+1\right)^{\frac{3}{2}} \boldsymbol{\phi}\right\|^{2}+\left\|\left(\mathcal{N}_{\perp}+1\right)^{2} \boldsymbol{\phi}\right\|^{2}\right),
\end{aligned}
$$


where we used the fact that $\mathrm{d} \Gamma_{\perp}(h)=\mathbb{K}_{0}$. Moreover, $\mathbb{K}_{0}^{\frac{1}{2}} \leq 1+\mathbb{K}_{0}=1+\mathbb{H}_{0}-\mathbb{K}_{1}-\mathbb{K}_{2}-\mathbb{K}_{2}^{*}$ implies

$$
\begin{aligned}
\left\|\mathbb{K}_{0}^{\frac{1}{2}}\left(\mathcal{N}_{\perp}+1\right)^{\frac{3}{2}} \boldsymbol{\phi}\right\|^{2} \leq & \left\|\left(\mathbb{H}_{0}+1\right)\left(\mathcal{N}_{\perp}+1\right)^{\frac{3}{2}} \boldsymbol{\phi}\right\|^{2} \\
& +\left|\left\langle\left(\mathcal{N}_{\perp}+1\right)^{\frac{3}{2}} \boldsymbol{\phi}, \mathbb{K}_{1}\left(\mathcal{N}_{\perp}+1\right)^{\frac{3}{2}} \boldsymbol{\phi}\right\rangle\right| \\
& +2\left|\left\langle\left(\mathcal{N}_{\perp}+1\right)^{\frac{3}{2}} \boldsymbol{\phi}, \mathbb{K}_{2}\left(\mathcal{N}_{\perp}+1\right)^{\frac{3}{2}} \boldsymbol{\phi}\right\rangle\right| \\
\leq & \mathbb{C}\left(\left\|\mathbb{H}_{0}\left(\mathcal{N}_{\perp}+1\right)^{\frac{3}{2}} \boldsymbol{\phi}\right\|+\left\|\left(\mathcal{N}_{\perp}+1\right)^{2} \boldsymbol{\phi}\right\|\right)^{2},
\end{aligned}
$$

where we used the fact that $\left|\left\langle\phi, \mathbb{K}_{j} \phi\right\rangle\right| \leq \mathbb{C}\left\|\left(\mathcal{N}_{\perp}+1\right)^{\frac{1}{2}} \phi\right\|^{2}$ for $j=1,2$ by the estimate (2.6).

Part (b). Since $\left[\mathbb{K}_{0}, \mathcal{N}_{\perp}\right]=\left[\mathbb{K}_{1}, \mathcal{N}_{\perp}\right]=\left[\mathbb{K}_{4}, \mathcal{N}_{\perp}\right]=0$, equation (2.40) implies that

$$
\begin{aligned}
{\left[\mathbb{H}_{\leq N},\left(\mathcal{N}_{\perp}+1\right)^{\ell}\right]=} & {\left[\mathbb{K}_{2},\left(\mathcal{N}_{\perp}+1\right)^{\ell}\right] g_{\mathcal{N}_{\perp}}+g_{\mathcal{N}_{\perp}}\left[\mathbb{K}_{2}^{*},\left(\mathcal{N}_{\perp}+1\right)^{\ell}\right] } \\
& +\left[\mathbb{K}_{3},\left(\mathcal{N}_{\perp}+1\right)^{\ell}\right] \widetilde{g}_{\mathcal{N}_{\perp}}+\widetilde{g}_{\mathcal{N}_{\perp}}\left[\mathbb{K}_{3}^{*},\left(\mathcal{N}_{\perp}+1\right)^{\ell}\right],
\end{aligned}
$$

where $g_{\mathcal{N}_{\perp}}:=\frac{\sqrt{\left[\left(N-\mathcal{N}_{\perp}\right)\left(N-\mathcal{N}_{\perp}-1\right)\right]_{+}}}{N-1}$ and $\widetilde{g}_{\mathcal{N}_{\perp}}:=\frac{\sqrt{\left[N-\mathcal{N}_{\perp}\right]_{+}}}{N-1}$. For $N \geq 2$,

$$
\left\|g_{\mathcal{N}_{\perp}} \phi\right\|_{\mathcal{F}_{\perp} \leq N} \leq 2\|\boldsymbol{\phi}\|_{\mathcal{F}_{\perp}^{\leq N}}, \quad\left\|\widetilde{g}_{\mathcal{N}_{\perp}} \phi\right\|_{\mathcal{F}_{\perp}^{\leq N}} \leq 3(N+1)^{-\frac{1}{2}}\|\boldsymbol{\phi}\|_{\mathcal{F}_{\perp} \leq N}
$$

By equation (4.1), we find that

$$
\left[\mathbb{K}_{2},\left(\mathcal{N}_{\perp}+1\right)^{\ell}\right]=-\mathbb{K}_{2}\left(\left(\mathcal{N}_{\perp}+3\right)^{\ell}-\left(\mathcal{N}_{\perp}+1\right)^{\ell}\right)
$$

and analogously for $\mathbb{K}_{2}^{*}, \mathbb{K}_{3}$ and $\mathbb{K}_{3}^{*}$. Since it holds for $a, k \geq 0$ and $c \geq 1$ that

$$
(k+a)^{c}-k^{c} \leq c a(k+a)^{c-1} \leq c a^{c}(k+1)^{c-1},
$$

we conclude with part (a) that

$$
\begin{aligned}
\left\|\left[\mathbb{K}_{2},\left(\mathcal{N}_{\perp}+1\right)^{\ell}\right] g_{\mathcal{N}_{\perp}} \phi\right\|_{\mathcal{F}_{\perp} \leq N} & \leq \mathbb{C}\left\|\left(\left(\mathcal{N}_{\perp}+3\right)^{\ell+1}-\left(\mathcal{N}_{\perp}+1\right)^{\ell+1}\right) g_{\mathcal{N}_{\perp}} \phi\right\|_{\mathcal{F}_{\perp} \leq N} \\
& \leq 3^{\ell} \mathfrak{C}\left\|\left(\mathcal{N}_{\perp}+1\right)^{\ell} \boldsymbol{\phi}\right\|_{\mathcal{F}_{\perp}^{\leq N}}, \\
\left\|\left[\mathbb{K}_{3},\left(\mathcal{N}_{\perp}+1\right)^{\ell}\right] \widetilde{g}_{\mathcal{N}_{\perp}} \phi\right\|_{\mathcal{F}_{\perp} \leq N} & \leq 3^{\ell} \ell \mathfrak{C}\left\|\left(\frac{\mathcal{N}_{\perp}+1}{N+1}\right)^{\frac{1}{2}}\left(\mathcal{N}_{\perp}+1\right)^{\ell} \boldsymbol{\phi}\right\|_{\mathcal{F}_{\perp}^{\leq N}} \\
& \leq 3^{\ell} \ell \mathfrak{C}\left\|\left(\mathcal{N}_{\perp}+1\right)^{\ell} \boldsymbol{\phi}\right\|_{\mathcal{F}_{\perp}^{\leq N}},
\end{aligned}
$$

and similarly for $\mathbb{K}_{2}^{*}$ and $\mathbb{K}_{3}^{*}$. The proof for $\mathbb{H}_{0}$ works analogously.

Next, we observe that the operators $\mathbb{H}_{j}$ and $\mathbb{R}_{j}$ can be bounded in terms of $\mathcal{N}_{\perp}$ and $\mathbb{H}_{0}$, which follows immediately from Lemma 5.2a:

Lemma 5.3. Let $\phi \in \mathcal{F}_{\perp}$ and $b \geq 0$.

(a) For any $j \in \mathbb{N}$, it holds that

$$
\begin{aligned}
& \left\|\left(\mathcal{N}_{\perp}+1\right)^{b} \mathbb{H}_{j} \phi\right\| \\
& \quad \leq \mathfrak{C}(b+j)\left(\left\|\left(\mathcal{N}_{\perp}+1\right)^{b+\frac{j}{2}+1} \phi\right\|+\left\|\left(\mathcal{N}_{\perp}+1\right)^{b} \mathbb{H}_{0}\left(\mathcal{N}_{\perp}+1\right)^{\frac{3}{2}} \phi\right\|\right) .
\end{aligned}
$$


(b) Further,

$$
\begin{aligned}
\left\|\left(\mathcal{N}_{\perp}+1\right)^{b} \mathbb{R}_{0}^{(1)} \phi\right\| \leq & \mathbb{C}(b)\left(\left\|\left(\mathcal{N}_{\perp}+1\right)^{b+\frac{3}{2}} \boldsymbol{\phi}\right\|\right. \\
& \left.+\lambda_{N}^{\frac{1}{2}}\left\|\left(\mathcal{N}_{\perp}+1\right)^{b+2} \boldsymbol{\phi}\right\|\right), \\
\left\|\left(\mathcal{N}_{\perp}+1\right)^{b} \mathbb{R}_{0} \phi\right\| \leq & \mathbb{C}(b)\left(\left\|\left(\mathcal{N}_{\perp}+1\right)^{b+\frac{3}{2}} \phi\right\|+\lambda_{N}^{\frac{1}{2}}\left\|\left(\mathcal{N}_{\perp}+1\right)^{b+2} \boldsymbol{\phi}\right\|\right. \\
& \left.+\lambda_{N}^{\frac{1}{2}}\left\|\left(\mathcal{N}_{\perp}+1\right)^{b} \mathbb{H}_{0}\left(\mathcal{N}_{\perp}+1\right)^{\frac{3}{2}} \boldsymbol{\phi}\right\|\right), \\
\left\|\left(\mathcal{N}_{\perp}+1\right)^{b} \mathbb{R}_{1} \phi\right\| \leq & \mathbb{C}(b)\left(\left\|\left(\mathcal{N}_{\perp}+1\right)^{b+2} \boldsymbol{\phi}\right\|+\lambda_{N}^{\frac{1}{2}}\left\|\left(\mathcal{N}_{\perp}+1\right)^{b+\frac{5}{2}} \boldsymbol{\phi}\right\|\right. \\
& \left.+\left\|\left(\mathcal{N}_{\perp}+1\right)^{b} \mathbb{H}_{0}\left(\mathcal{N}_{\perp}+1\right)^{\frac{3}{2}} \phi\right\|\right),
\end{aligned}
$$

and for any $j \in \mathbb{N}_{0}$,

$$
\begin{aligned}
\left\|\left(\mathcal{N}_{\perp}+1\right)^{b} \mathbb{R}_{j} \phi\right\| \leq & \mathfrak{C}(b, j)\left(\left\|\left(\mathcal{N}_{\perp}+1\right)^{b+\frac{j+3}{2}} \phi\right\|+\lambda_{N}^{\frac{1}{2}}\left\|\left(\mathcal{N}_{\perp}+1\right)^{b+\frac{j+4}{2}} \phi\right\|\right. \\
& \left.+\left\|\left(\mathcal{N}_{\perp}+1\right)^{b} \mathbb{H}_{0}\left(\mathcal{N}_{\perp}+1\right)^{\frac{3}{2}} \phi\right\|\right)
\end{aligned}
$$

\subsubsection{Bound for $\mathbb{A}_{\text {red }}^{(m)}$}

In this section, we show that $\mathbb{A}_{\text {red }}^{(m)}$ as in definition (3.57) is bounded in terms of $\mathbb{H}_{0}$ and $\mathcal{N}_{\perp}$.

Lemma 5.4. For $A^{(m)}$ satisfying the bound (3.11) and the corresponding operator $\mathbb{A}_{\text {red }}^{(m)}$ as in definition (3.57), it holds that

$$
\left\|\mathbb{A}_{\text {red }}^{(m)} \phi\right\| \leq \mathfrak{C}(m) N^{-\frac{1}{2}}\left(\left\|\left(\mathcal{N}_{\perp}+1\right) \phi\right\|+\left\|\mathbb{H}_{0} \phi\right\|\right)
$$

Proof. In the following, we abbreviate

$$
\psi_{N}:=U_{N, \varphi}^{*} \phi
$$

Decomposing $1=p_{j_{1}} \cdots p_{j_{m}}+\left(1-p_{j_{1}} \cdots p_{j_{m}}\right)$ and observing that

$$
p_{j_{1}} \cdots p_{j_{m}} A_{j_{1}, \ldots, j_{m}}^{(m)} p_{j_{1}} \cdots p_{j_{m}}=\langle A\rangle^{(m)} p_{j_{1}} \cdots p_{j_{m}}
$$

yields

$$
\begin{aligned}
& \left\|\mathbb{A}_{\text {red }}^{(m)} \phi\right\| \leq\left(\begin{array}{c}
N \\
m
\end{array}\right)^{-1}\left\|\langle A\rangle^{(m)} \sum_{1 \leq j_{1}<\cdots<j_{m} \leq N}\left(1-p_{j_{1}} \cdots p_{j_{m}}\right) \psi_{N}\right\|_{\mathfrak{Y}^{N}} \\
& +\left(\begin{array}{l}
N \\
m
\end{array}\right)^{-1}\left\|\sum_{1 \leq j_{1}<\cdots<j_{m} \leq N} A_{j_{1}, \ldots, j_{m}}^{(m)}\left(1-p_{j_{1}} \cdots p_{j_{m}}\right) \psi_{N}\right\|_{\mathfrak{G}^{N}} \\
& +\left(\begin{array}{l}
N \\
m
\end{array}\right)^{-1}\left\|\sum_{\substack{1 \leq j_{1}<\cdots<j_{m} \leq N \\
\text { ambridge University Press }}}\left(1-p_{j_{1}} \cdots p_{j_{m}}\right) A_{j_{1}, \ldots, j_{m}}^{(m)} p_{j_{1}} \cdots p_{j_{m}} \psi_{N}\right\|_{\mathfrak{S}^{N}} .
\end{aligned}
$$


To estimate the contributions in inequality (5.51), observe first that

$$
\left\|A^{(m)} \varphi^{\otimes m}\right\|_{\mathfrak{Y}^{m}} \leq \mathfrak{C}
$$

by Lemma 5.1a, because $h \varphi=0$. Further, it was shown in [10, Lemma 3.2] that

$$
\left\|q_{1} \cdots q_{\ell} \psi_{N}\right\|_{\mathfrak{H}^{N}} \leq\left(N^{-\ell}+2^{\ell} N^{-\ell}\left\langle\boldsymbol{\phi}, \mathcal{N}_{\perp}^{\ell} \boldsymbol{\phi}\right\rangle_{\mathcal{F}_{\perp}^{\leq N}}\right)^{\frac{1}{2}} \leq N^{-\frac{1}{2}} 2^{\frac{\ell}{2}}\left\|\left(\mathcal{N}_{\perp}+1\right)^{\frac{1}{2}} \boldsymbol{\phi}\right\|_{\mathcal{F}_{\perp}^{\leq N}}
$$

for $\ell \in\{1, \ldots, N\}$, because $\frac{\mathcal{N}_{\perp}}{N} \leq 1$ as operator on $\mathcal{F}_{\perp}^{\leq N}$. Hence, by the permutation symmetry of $\psi_{N}$, it holds that

$$
\begin{aligned}
(5.51 a) & \leq \mathfrak{C}\left\|\left(1-p_{1} \cdots p_{m}\right) \psi_{N}\right\|_{\mathfrak{S}^{N}} \\
& \leq \mathfrak{C} \sum_{\ell=1}^{m}\left(\begin{array}{c}
m \\
\ell
\end{array}\right)\left\|q_{1} \cdots q_{\ell} p_{\ell+1} \cdots p_{m} \psi_{N}\right\|_{\mathfrak{S}^{N}} \\
& \leq \mathfrak{C}(m) N^{-\frac{1}{2}}\left\|\left(\mathcal{N}_{\perp}+1\right)^{\frac{1}{2}} \boldsymbol{\phi}\right\|_{\mathcal{F}_{\perp}^{\leq N}} .
\end{aligned}
$$

For expression (5.51b), Lemma 5.1a implies

$$
\begin{aligned}
(5.51 b) & \leq\left\|A_{1, \ldots, m}^{(m)}\left(1-p_{1} \cdots p_{m}\right) \psi_{N}\right\|_{\mathfrak{S}^{N}} \\
& \leq \mathbb{C}\left(\left\|\sum_{j=1}^{m} h_{j}\left(1-p_{1} \cdots p_{m}\right) \psi_{N}\right\|_{\mathfrak{S}^{N}}+\left\|\left(1-p_{1} \cdots p_{m}\right) \psi_{N}\right\|_{\mathfrak{S}^{N}}\right) \\
& \leq \mathbb{C}(m)\left(\left\|\sum_{j=1}^{m} h_{j} \psi_{N}\right\|_{\mathfrak{S}^{N}}+N^{-\frac{1}{2}}\left\|\left(\mathcal{N}_{\perp}+1\right)^{\frac{1}{2}} \boldsymbol{\phi}\right\|_{\mathcal{F}_{\perp}^{\leq N}}\right),
\end{aligned}
$$

since $h_{j}=q_{j} h_{j} q_{j}$ and $q_{j}\left(1-p_{1} \cdots p_{m}\right)=q_{j}$. For the first term, Lemma 5.1b yields

$$
\left\|\sum_{j=1}^{m} h_{j} \psi_{N}\right\|_{\mathfrak{T}^{N}} \leq \sqrt{\frac{m}{N}}\left\|\sum_{j=1}^{N} h_{j} \psi_{N}\right\| \leq \mathfrak{C}(m) N^{-\frac{1}{2}}\left(\left\|\mathbb{H}_{0} \boldsymbol{\phi}\right\|_{\mathcal{F}_{\perp}^{\leq N}}+\left\|\left(\mathcal{N}_{\perp}+1\right) \boldsymbol{\phi}\right\|_{\mathcal{F}_{\perp}^{\leq N}}\right)
$$

because $h \varphi=0$ implies that $\mathbb{K}_{0}=\mathrm{d} \Gamma_{\perp}(h)=U_{N, \varphi}^{*} \mathbb{K}_{0} U_{N, \varphi}$ and, by Lemma 5.2a,

$$
\left\|\mathbb{H}_{0} \phi\right\|_{\mathcal{F}_{\perp}} \geq\left\|\mathbb{K}_{0} \phi\right\|-\mathfrak{C}\left\|\left(\mathcal{N}_{\perp}+1\right) \phi\right\|_{\mathcal{F}_{\perp}} .
$$


Finally, for $m \ll N$,

$$
\begin{aligned}
\left(\begin{array}{l}
N \\
m
\end{array}\right) & (5.51 c))^{2} \\
= & \left(\begin{array}{l}
N \\
m
\end{array}\right) \sum_{1 \leq j_{1}<\cdots<j_{m} \leq N}\left\langle\psi_{N}, p_{1} \cdots p_{m} A_{1, \ldots, m}^{(m)}\left(1-p_{1} \cdots p_{m}\right)\left(1-p_{j_{1}} \cdots p_{j_{m}}\right)\right. \\
& \left.\times A_{j_{1}, \ldots, j_{m}}^{(m)} p_{j_{1}} \cdots p_{j_{m}} \psi_{N}\right\rangle_{\mathfrak{S}^{N}} \\
= & \left(\begin{array}{l}
N \\
m
\end{array}\right) \sum_{\ell=0}^{m}\left(\begin{array}{c}
N-m \\
\ell
\end{array}\right)\left(\begin{array}{c}
m \\
\ell
\end{array}\right)\left\langle\psi_{N}, p_{1} \cdots p_{m} A_{1, \ldots, m}^{(m)}\left(1-p_{1} \cdots p_{m}\right)\left(1-p_{\ell+1} \cdots p_{\ell+m}\right)\right. \\
& \left.\times A_{\ell+1, \ldots, \ell+m}^{(m)} p_{\ell+1} \cdots p_{\ell+m} \psi_{N}\right\rangle_{\mathfrak{Y}^{N}} \\
\leq & \left(\begin{array}{l}
N \\
m
\end{array}\right) \sum_{\ell=0}^{m-1}\left(\begin{array}{c}
N-m \\
\ell
\end{array}\right)\left(\begin{array}{c}
m \\
\ell
\end{array}\right)\left\|A_{1, \ldots, m}^{(m)} \varphi^{\otimes m}\right\|_{\mathfrak{Y}^{m}}^{2}\|\boldsymbol{\phi}\|_{\mathcal{F}_{\perp}^{\leq N}}^{2} \\
& +\left(\begin{array}{l}
N \\
m
\end{array}\right)\left(\begin{array}{c}
N-m \\
m
\end{array}\right)\left\langle\psi_{N}, p_{1} \cdots p_{m} A_{1, \ldots, m}^{(m)}\left(1-p_{1} \cdots p_{m}\right)\left(1-p_{m+1} \cdots p_{2 m}\right)\right. \\
& \left.\times A_{m+1, \ldots, 2 m}^{(m)} p_{m+1} \cdots p_{2 m} \psi_{N}\right\rangle_{\mathfrak{S}^{N}} \\
\leq & \mathbb{C}(m)\left(\begin{array}{c}
N \\
m
\end{array}\right)^{2} N^{-1}\left(\|\boldsymbol{\phi}\|_{\mathcal{F}_{\perp} \leq N}^{2}+\left\|\left(\mathcal{N}_{\perp}+1\right)^{\frac{1}{2}} \boldsymbol{\phi}\right\|_{\mathcal{F}_{\perp}^{\leq N}}^{2}\right)
\end{aligned}
$$

where we use the facts that

$$
\left(\begin{array}{l}
N \\
m
\end{array}\right) \sum_{\ell=0}^{m-1}\left(\begin{array}{c}
N-m \\
\ell
\end{array}\right)\left(\begin{array}{c}
m \\
\ell
\end{array}\right) \leq m 2^{m}\left(\begin{array}{l}
N \\
m
\end{array}\right)^{2} \frac{\left(\begin{array}{c}
N-m \\
m-1
\end{array}\right)}{\left(\begin{array}{c}
N \\
m
\end{array}\right)} \leq \mathbb{C}(m)\left(\begin{array}{l}
N \\
m
\end{array}\right)^{2} N^{-1}
$$

and

$$
\begin{aligned}
\left\langle\psi_{N},\right. & \left.p_{1} \cdots p_{m} A_{1, \ldots, m}^{(m)}\left(1-p_{1} \cdots p_{m}\right)\left(1-p_{m+1} \cdots p_{2 m}\right) A_{m+1, \ldots, 2 m}^{(m)} p_{m+1} \cdots p_{2 m} \psi_{N}\right\rangle_{\mathfrak{H}^{N}} \\
= & \left\langle\left(1-p_{m+1} \cdots p_{2 m}\right) \psi_{N}, p_{1} \cdots p_{m} A_{1, \ldots, m}^{(m)} A_{m+1, \ldots, 2 m}^{(m)} p_{m+1} \cdots p_{2 m}\right. \\
& \left.\times\left(1-p_{1} \cdots p_{m}\right) \psi_{N}\right\rangle_{\mathfrak{S}^{N}} \\
\leq & \left\|A_{1, \ldots, m}^{(m)} \varphi^{\otimes m}\right\|_{\mathfrak{S}^{m}}^{2}\left\|\left(1-p_{1} \cdots p_{m}\right) \psi_{N}\right\|_{\mathfrak{H}^{N}}^{2} \\
\leq & \mathbb{C}(m)\left(N^{-\frac{1}{2}}\left\|\left(\mathcal{N}_{\perp}+1\right)^{\frac{1}{2}} \phi\right\|_{\mathcal{F}_{\perp}^{\leq N}}\right)^{2}
\end{aligned}
$$

as in estimate (5.54).

\subsubsection{Resolvent estimates}

Lemma 5.5. Let

$$
\mathbb{I}^{(n)} \in\left\{\mathbb{1}, \mathbb{P}_{0}^{(n)}, \mathbb{Q}_{0}^{(n)}\right\}
$$

and $z \in \gamma^{(n)}$.

(a) It holds that

$$
\left\|\frac{\mathbb{I}^{(n)}}{z-\mathbb{H}_{0}}\right\|_{\mathcal{L}\left(\mathcal{F}_{\perp}\right)} \leq \mathfrak{C}(n)
$$


and for sufficiently large $N$,

$$
\left\|\frac{1}{z-\mathbb{H}<}\right\|_{\mathcal{L}\left(\mathcal{F}_{\perp}\right)} \leq \mathfrak{C}(n) .
$$

(b) Let $b \geq 0$. Then

$$
\begin{aligned}
\left\|\left(\mathcal{N}_{\perp}+1\right)^{b+1} \frac{\mathbb{I}^{(n)}}{z-\mathbb{H}_{0}} \phi\right\| & \leq \mathfrak{C}(n, b)\left\|\left(\mathcal{N}_{\perp}+1\right)^{b} \phi\right\|, \\
\left\|\left(\mathcal{N}_{\perp}+1\right)^{b} \mathbb{H}_{0} \frac{\mathbb{I}^{(n)}}{z-\mathbb{H}_{0}} \phi\right\| & \leq \mathfrak{C}(n, b)\left\|\left(\mathcal{N}_{\perp}+1\right)^{b} \phi\right\| .
\end{aligned}
$$

Proof. By definition (3.33) of $\mathfrak{g}^{(n)}$, it follows that

$$
\inf _{\substack{z \in \gamma^{(n)} \\ \lambda \in \sigma\left(\mathbb{H}_{0}\right)}}|z-\lambda|=\min \left\{\left|z-E_{0}^{(n)}\right|,\left|z-E_{0}^{(n-1)}\right|,\left|z-E_{0}^{(n+1)}\right|\right\}=\mathfrak{g}^{(n)},
$$

which implies the first part of (a), and the second part follows with Lemma 4.8a. For part (b), recall that there exists a Bogoliubov transformation $\mathbb{U}_{\mathcal{V}_{0}}$ diagonalising $\mathbb{H}_{0}$ (Lemma 4.7a), hence

$$
\left[\mathbb{U}_{\mathcal{V}_{0}}^{*}\left(\mathcal{N}_{\perp}+1\right) \mathbb{U}_{\mathcal{V}_{0}}, \mathbb{H}_{0}\right]=0, \quad\left[\mathbb{U}_{\mathcal{V}_{0}} \frac{\mathbb{I}^{(n)}}{z-\mathbb{H}_{0}} \mathbb{U}_{\mathcal{V}_{0}}^{*}, \mathcal{N}_{\perp}\right]=0
$$

As a consequence, Lemma 4.7 e implies that

$$
\mathbb{U}_{\mathcal{V}_{0}}^{*}\left(\mathcal{N}_{\perp}+1\right)^{k} \mathbb{U}_{\mathcal{V}_{0}} \leq \mathfrak{C}^{k}\left(\mathbb{H}_{0}-E_{0}^{(0)}+1\right)^{k},
$$

hence

$$
\begin{aligned}
\frac{\mathbb{I}^{(n)}}{\bar{z}-\mathbb{H}_{0}} \mathbb{U}_{\mathcal{V}_{0}}^{*}\left(\mathcal{N}_{\perp}+1\right)^{2} \mathbb{U}_{\mathcal{V}_{0}} \frac{\mathbb{I}^{(n)}}{z-\mathbb{H}_{0}} & \leq \mathfrak{C}^{2}\left|\frac{\mathbb{I}^{(n)}}{z-\mathbb{H}_{0}}\right|^{2}\left(\left|\mathbb{H}_{0}-z\right|+\left|z-E_{0}^{(0)}+1\right|\right)^{2} \\
& \leq \mathfrak{C}(n)^{2},
\end{aligned}
$$

because $\left|z-E_{0}^{(0)}+1\right| \leq\left|E_{0}^{(n)}\right|+\mathfrak{g}^{(n)}+\left|E_{0}^{(0)}\right|+1 \leq \mathfrak{C}(n)$. Consequently, Lemma 4.4 leads, for $b \geq 1$, to the estimate

$$
\begin{aligned}
\left\|\left(\mathcal{N}_{\perp}+1\right)^{b} \frac{\mathbb{I}^{(n)}}{z-\mathbb{H}_{0}} \boldsymbol{\phi}\right\| & =\left\|\left(\mathcal{N}_{\perp}+1\right)^{b} \mathbb{U}_{\mathcal{V}_{0}^{*}}^{*} \mathbb{U}_{\mathcal{V}_{0}} \frac{\mathbb{I}^{(n)}}{z-\mathbb{H}_{0}} \mathbb{U}_{\mathcal{V}_{0}}^{*} \mathbb{U}_{\mathcal{V}_{0}} \phi\right\| \\
& \leq \mathfrak{C}(b)\left\|\left(\mathcal{N}_{\perp}+1\right)\left(\mathcal{N}_{\perp}+1\right)^{b-1} \mathbb{U}_{\mathcal{V}_{0}} \frac{\mathbb{I}^{(n)}}{z-\mathbb{H}_{0}} \mathbb{U}_{\mathcal{V}_{0}}^{*} \mathbb{U}_{\mathcal{V}_{0}} \phi\right\| \\
& =\mathfrak{C}(b)\left\|\left(\mathcal{N}_{\perp}+1\right) \mathbb{U}_{\mathcal{V}_{0}} \frac{\mathbb{I}^{(n)}}{z-\mathbb{H}_{0}} \mathbb{U}_{\mathcal{V}_{0}}^{*}\left(\mathcal{N}_{\perp}+1\right)^{b-1} \mathbb{U}_{\mathcal{V}_{0}} \phi\right\| \\
& \leq \mathfrak{C}(n, b)\left\|\left(\mathcal{N}_{\perp}+1\right)^{b-1} \mathbb{U}_{\mathcal{V}_{0}} \phi\right\| \\
& \leq \mathfrak{C}(n, b)\left\|\left(\mathcal{N}_{\perp}+1\right)^{b-1} \boldsymbol{\phi}\right\| .
\end{aligned}
$$

The second statement of (b) is a consequence of the triangle inequality, since

$$
\mathbb{H}_{0} \frac{\mathbb{I}^{(n)}}{z-\mathbb{H}_{0}}=-\mathbb{I}^{(n)}+z \frac{\mathbb{I}^{(n)}}{z-\mathbb{H}_{0}} .
$$


5.2.4. Bounds for moments of $\mathcal{N}_{\perp}$ and $\mathbb{K}_{4}$ with respect to $\mathbb{P}^{(n)}$

In this section, we show that moments of $\mathcal{N}_{\perp}$ with respect to both $\chi^{(n)}$ and $\mathbb{K}_{4} \chi^{(n)}$ are bounded uniformly in $N$.

Lemma 5.6. Let $\chi^{(n)} \in \mathfrak{E}^{(n)}$ and $b \geq 0$. Then

(a)

$$
\left\langle\chi^{(n)},\left(\mathcal{N}_{\perp}+1\right)^{b} \chi^{(n)}\right\rangle \leq \mathfrak{C}(n, b)
$$

(b)

$$
\left\|\left(\mathcal{N}_{\perp}+1\right)^{b} \mathbb{K}_{4} \chi^{(n)}\right\| \leq \mathfrak{C}(n, b)
$$

Proof. Part (a). Proposition 3.14 with $a=0$ implies that

$$
\begin{aligned}
\operatorname{Tr} & \left.\mathbb{P}^{(n)}\left(\mathcal{N}_{\perp}+1\right)^{b+1}\right) \\
= & \operatorname{Tr}\left(\mathbb{P}_{0}^{(n)}\left(\mathcal{N}_{\perp}+1\right)^{b+1}\right) \\
& +\lambda_{N}^{\frac{1}{2}} \operatorname{Tr}\left(\frac{1}{2 \pi \mathrm{i}} \oint_{\gamma^{(n)}} \frac{1}{z-E_{0}^{(n)}} \frac{\mathbb{Q}^{(n)}}{z-\mathbb{H}^{<}} \mathbb{R}_{0} \mathbb{P}_{0}^{(n)}\left(\mathcal{N}_{\perp}+1\right)^{b+1} \mathrm{~d} z\right) \\
& +\lambda_{N}^{\frac{1}{2}} \operatorname{Tr}\left(\frac{1}{2 \pi \mathrm{i}} \oint_{\gamma^{(n)}} \frac{\mathbb{P}^{(n)}}{z-\mathbb{H}^{<}} \mathbb{R}_{0} \frac{1}{z-\mathbb{H}_{0}}\left(\mathcal{N}_{\perp}+1\right)^{b+1} \mathrm{~d} z\right) .
\end{aligned}
$$

For the first term, note that $(5.70 a) \leq \mathfrak{C}(n, b)$ by Lemma 4.7d. Denoting by $\left\{\chi_{0}^{(n, m)}\right\}_{m=1}^{\delta_{0}^{(n)}}$ some orthonormal basis of $\mathfrak{E}_{0}^{(n)}$ and interchanging trace and integral by Fubini's theorem, we estimate the second term as

$$
\begin{aligned}
& |(5.70 b)| \\
& \quad \leq \lambda_{N}^{\frac{1}{2}} \mathfrak{g}^{(n)} \sup _{z \in \gamma^{(n)}}\left(\left|\frac{1}{z-E_{0}^{(n)}}\right| \sum_{m=1}^{\delta_{0}^{(n)}}\left\|\left(\mathcal{N}_{\perp}+1\right)^{b+1} \chi_{0}^{(n, m)}\right\|\left\|\frac{\mathbb{Q}^{(n)}}{z-\mathbb{H}^{<}}\right\|\left\|_{\mathrm{op}}\right\| \mathbb{R}_{0} \chi_{0}^{(n, m)} \|\right) \\
& \quad \leq N^{-\frac{1}{2}} \mathfrak{C}(n, b)
\end{aligned}
$$

by Lemmas $4.7 \mathrm{~d}, 5.5 \mathrm{a}, 5.3 \mathrm{~b}$ and $5.2 \mathrm{~b}$ and since $\mathbb{H}_{0} \chi_{0}^{(n, m)}=E_{0}^{(n)} \chi_{0}^{(n, m)}$. Similarly, we find for the last term

$$
\begin{aligned}
& |(5.70 c)| \\
& \left.\leq \lambda_{N}^{\frac{1}{2}} \mathfrak{g}^{(n)} \sup _{\substack{z \in \gamma^{(n)} \\
v \in \iota^{(n)}}}\left(\left|\frac{1}{z-E^{(v)}}\right| \sum_{m=1}^{\delta_{0}^{(n)}} \mid\left\langle\chi^{(n, m)}, \mathbb{R}_{0} \frac{1}{z-\mathbb{H}_{0}}\left(\mathcal{N}_{\perp}+1\right)^{b+1} \chi^{(n, m)}\right\rangle\right)\right) \\
& \leq \mathbb{C}(n) N^{-\frac{1}{2}} \sum_{m=1}^{\delta_{0}^{(n)}}\left|\left\langle\chi^{(n, m)}, \mathbb{R}_{0}^{(1)} \frac{1}{z-\mathbb{H}_{0}}\left(\mathcal{N}_{\perp}+1\right)^{b+1} \chi^{(n, m)}\right\rangle\right| \\
& \quad+\mathbb{C}(n) N^{-1} \sum_{m=1}^{\delta_{0}^{(n)}}\left|\left\langle\chi^{(n, m)}, \mathbb{K}_{4} \frac{1}{z-\mathbb{H}_{0}}\left(\mathcal{N}_{\perp}+1\right)^{b+1} \chi^{(n, m)}\right\rangle\right|
\end{aligned}
$$


for $\left\{\chi^{(n, m)}\right\}_{m=1}^{\delta_{0}^{(n)}}$ some orthonormal basis of $\mathfrak{E}^{(n)}$ and for $\mathbb{R}_{0}=\mathbb{R}_{0}^{(1)}+\lambda_{N}^{\frac{1}{2}} \mathbb{K}_{4}$ as defined in Proposition 3.12. In expression $(5.72 a)$, we obtain the bound

$$
\begin{aligned}
& \|\left\langle\chi^{(n, m)},\left(\mathcal{N}_{\perp}+1\right)^{b+1} \frac{1}{z-\mathbb{H}_{0}} \mathbb{R}_{0}^{(1)} \chi^{(n, m)}\right\rangle \\
& \leq\left\|\left(\mathcal{N}_{\perp}+1\right)^{\frac{b}{2}} \chi^{(n, m)}\right\|\left\|\left(\mathcal{N}_{\perp}+1\right)^{\frac{b}{2}+1} \frac{1}{z-\mathbb{H}_{0}} \mathbb{R}_{0}^{(1)} \chi^{(n, m)}\right\| \\
& \leq \mathbb{C}(n, b)\left\|\left(\mathcal{N}_{\perp}+1\right)^{\frac{b}{2}} \chi^{(n, m)}\right\|\left(\left\|\left(\mathcal{N}_{\perp}+1\right)^{\frac{b+3}{2}} \chi^{(n, m)}\right\|\right. \\
& \left.\quad+N^{-\frac{1}{2}}\left\|\left(\mathcal{N}_{\perp}+1\right)^{\frac{b+4}{2}} \chi^{(n, m)}\right\|\right)
\end{aligned}
$$

by Lemmas $5.5 b$ and $5.3 b$. Since

$$
\left\|\left(\mathcal{N}_{\perp}+1\right)^{\frac{b+\ell}{2}} \chi^{(n, m)}\right\| \leq \mathfrak{C}(n, b+\ell) N^{\frac{\ell}{6}}\left\|\left(\mathcal{N}_{\perp}+1\right)^{\frac{b}{2}} \chi^{(n, m)}\right\|
$$

for all $\ell \in \mathbb{N}_{0}$ by Lemma 4.8 c, it follows that

$$
(5.72 a) \leq \mathfrak{C}(n, b)\left\|\left(\mathcal{N}_{\perp}+1\right)^{\frac{b}{2}} \chi^{(n, m)}\right\|^{2} .
$$

Since $\left[\mathbb{K}_{4}, \mathcal{N}_{\perp}\right]=0$, the sum in expression $(5.72 b)$ can be estimated as

$$
\begin{aligned}
\|\left\langle\chi^{(n, m)}, \mathbb{K}_{4} \frac{1}{z-\mathbb{H}_{0}}\left(\mathcal{N}_{\perp}+1\right)^{b+1} \chi^{(n, m)}\right\rangle \\
\leq\left\|\left(\mathcal{N}_{\perp}+1\right)^{\frac{b}{2}} \chi^{(n, m)}\right\|\left\|\mathbb{K}_{4}\left(\mathcal{N}_{\perp}+1\right)^{-\frac{b}{2}} \frac{1}{z-\mathbb{H}_{0}}\left(\mathcal{N}_{\perp}+1\right)^{b+1} \chi^{(n, m)}\right\| \\
\leq \mathbb{C}\left\|\left(\mathcal{N}_{\perp}+1\right)^{\frac{b}{2}} \chi^{(n, m)}\right\|\left(\left\|\mathbb{H}_{0}\left(\mathcal{N}_{\perp}+1\right)^{\frac{3-b}{2}} \frac{1}{z-\mathbb{H}_{0}}\left(\mathcal{N}_{\perp}+1\right)^{b+1} \chi^{(n, m)}\right\|\right. \\
\left.\quad+\left\|\left(\mathcal{N}_{\perp}+1\right)^{2-\frac{b}{2}} \frac{1}{z-\mathbb{H}_{0}}\left(\mathcal{N}_{\perp}+1\right)^{b+1} \chi^{(n, m)}\right\|\right) \\
\leq N^{\frac{5}{6}} \mathfrak{C}(n, b)\left\|\left(\mathcal{N}_{\perp}+1\right)^{\frac{b}{2}} \chi^{(n, m)}\right\|^{2} \\
\quad+\mathbb{C}(n, b)\left\|\left(\mathcal{N}_{\perp}+1\right)^{\frac{b}{2}} \chi^{(n, m)}\right\|\left\|\left(\mathcal{N}_{\perp}+1\right)^{2-\frac{b}{2}} \frac{1}{z-\mathbb{H}_{0}}\left(\mathcal{N}_{\perp}+1\right)^{b+1} \chi^{(n, m)}\right\|,
\end{aligned}
$$

where we use Lemmas 5.2a and 5.5b, the bound (5.74) and the fact that

$$
\begin{aligned}
& \left\|\mathbb{H}_{0}\left(\mathcal{N}_{\perp}+1\right)^{\frac{3-b}{2}} \frac{1}{z-\mathbb{H}_{0}} \phi\right\| \\
& \leq\left\|\left(\mathcal{N}_{\perp}+1\right)^{\frac{3-b}{2}} \phi\right\|+\left\|\left[\mathbb{H}_{0},\left(\mathcal{N}_{\perp}+1\right)^{\frac{3-b}{2}}\right] \frac{1}{z-\mathbb{H}_{0}} \phi\right\| \\
& \quad+|z|\left\|\left(\mathcal{N}_{\perp}+1\right)^{\frac{3-b}{2}} \frac{1}{z-\mathbb{H}_{0}} \phi\right\| \\
& \leq \mathbb{C}(n, b)\left(\left\|\left(\mathcal{N}_{\perp}+1\right)^{\frac{3-b}{2}} \phi\right\|+\left\|\left(\mathcal{N}_{\perp}+1\right)^{\frac{3-b}{2}} \frac{1}{z-\mathbb{H}_{0}} \phi\right\|\right)
\end{aligned}
$$


by Lemma 5.2b. To control expression (5.76b), we prove by induction that

$$
\begin{aligned}
& \left\|\left(\mathcal{N}_{\perp}+1\right)^{2-\frac{b}{2}} \frac{1}{z-\mathbb{H}_{0}}\left(\mathcal{N}_{\perp}+1\right)^{b+1} \phi\right\| \\
& \quad \leq \mathfrak{C}(n, b)\left\|\left(\mathcal{N}_{\perp}+1\right)^{\frac{b}{2}} \phi\right\|^{1-\left(\frac{1}{2}\right)^{k}}\left\|\left(\mathcal{N}_{\perp}+1\right)^{-\frac{b}{2}+2^{k+1}} \frac{1}{z-\mathbb{H}_{0}}\left(\mathcal{N}_{\perp}+1\right)^{b+1} \phi\right\|^{\left(\frac{1}{2}\right)^{k}}
\end{aligned}
$$

for all $k \in \mathbb{N}_{0}$. The base case $k=0$ is obvious. Now assume that inequality (5.78) holds for some $k \in \mathbb{N}_{0}$. Then

$$
\begin{aligned}
&\left\|\left(\mathcal{N}_{\perp}+1\right)^{2-\frac{b}{2}} \frac{1}{z-\mathbb{H}_{0}}\left(\mathcal{N}_{\perp}+1\right)^{b+1} \phi\right\| \\
& \leq \mathbb{C}(n, b)\left\|\left(\mathcal{N}_{\perp}+1\right)^{\frac{b}{2}} \phi\right\|^{1-\left(\frac{1}{2}\right)^{k}}\left\langle\left(\mathcal{N}_{\perp}+1\right)^{\frac{b}{2}} \boldsymbol{\phi},\left(\mathcal{N}_{\perp}+1\right)^{\frac{b}{2}+1} \frac{1}{z-\mathbb{H}_{0}}\right. \\
&\left.\times\left(\mathcal{N}_{\perp}+1\right)^{-b+2^{k+2}} \frac{1}{z-\mathbb{H}_{0}}\left(\mathcal{N}_{\perp}+1\right)^{b+1} \boldsymbol{\phi}\right)^{\left(\frac{1}{2}\right)^{k+1}} \\
& \leq \mathbb{C}(n, b)\left\|\left(\mathcal{N}_{\perp}+1\right)^{\frac{b}{2}} \boldsymbol{\phi}\right\|^{1-\left(\frac{1}{2}\right)^{k+1}} \\
& \quad \times\left\|\left(\mathcal{N}_{\perp}+1\right)^{-\frac{b}{2}+2^{k+2}} \frac{1}{z-\mathbb{H}_{0}}\left(\mathcal{N}_{\perp}+1\right)^{b+1} \phi\right\|^{\left(\frac{1}{2}\right)^{k+1}}
\end{aligned}
$$

by Lemma 5.5b. Now choose $k$ in inequality (5.78) such that $2^{k+2} \geq b+2$; then $-\frac{b}{2}+2^{k+1} \geq 1$ and consequently

$$
\begin{aligned}
& \left\|\left(\mathcal{N}_{\perp}+1\right)^{-\frac{b}{2}+2^{k+1}} \frac{1}{z-\mathbb{H}_{0}}\left(\mathcal{N}_{\perp}+1\right)^{b+1} \phi\right\| \\
& \leq \mathbb{C}(n, b)\left\|\left(\mathcal{N}_{\perp}+1\right)^{\frac{b+2^{k+2}}{2}} \phi\right\|^{\left(\frac{1}{2}\right)^{k}} \\
& \leq \mathbb{C}(n, b) N^{\frac{2}{3}}\left\|\left(\mathcal{N}_{\perp}+1\right)^{\frac{b}{2}} \chi^{(n, m)}\right\|^{\frac{1}{2^{k}}}
\end{aligned}
$$

by Lemma $5.5 b$ and inequality (5.74). In summary,

$$
\operatorname{Tr}\left(\mathbb{P}^{(n)}\left(\mathcal{N}_{\perp}+1\right)^{b+1}\right) \leq \mathfrak{C}(n, b)\left\|\left(\mathcal{N}_{\perp}+1\right)^{\frac{b}{2}} \chi^{(n, m)}\right\|^{2}
$$

Finally, we prove the lemma via the following bootstrap argument:

(1) Lemma $4.8 \mathrm{c}$ implies that

$$
\left\|\left(\mathcal{N}_{\perp}+1\right)^{\frac{1}{2}} \chi^{(n, m)}\right\| \leq \mathfrak{C}(n) \stackrel{(5.81)}{\longrightarrow} \operatorname{Tr}\left(\mathbb{P}^{(n)}\left(\mathcal{N}_{\perp}+1\right)^{2}\right) \leq \mathfrak{C}(n) .
$$

(2) By step (1),

$$
\left\|\left(\mathcal{N}_{\perp}+1\right) \chi^{(n, m)}\right\| \leq \mathfrak{C}(n) \stackrel{(5.81)}{\Longrightarrow} \operatorname{Tr}\left(\mathbb{P}^{(n)}\left(\mathcal{N}_{\perp}+1\right)^{3}\right) \leq \mathfrak{C}(n) .
$$

(b) By step $(b-1)$,

$$
\left\|\left(\mathcal{N}_{\perp}+1\right)^{\frac{b}{2}} \chi^{(n, m)}\right\| \leq \mathfrak{C}(n, b) \stackrel{(5.81)}{\Longrightarrow} \operatorname{Tr}\left(\mathbb{P}^{(n)}\left(\mathcal{N}_{\perp}+1\right)^{b+1}\right) \leq \mathfrak{C}(n, b) .
$$


Part (b). Define

$$
\mathbb{K}_{4}^{-}:=\left.\mathbb{K}_{4}\right|_{\mathcal{F} \leq N} \oplus 0
$$

By Lemma 5.2a and Assumption 3, there exists a constant $c$ such that

$$
\begin{aligned}
&\left\|\left(\mathcal{N}_{\perp}+1\right)^{b} \mathbb{K}_{4}^{-} \phi\right\|^{2} \\
&=\left\|\left(\mathcal{N}_{\perp}+1\right)^{b} \mathbb{K}_{4} \phi\right\|_{\mathcal{F}_{\perp}^{\leq N}}^{2} \\
& \leq \mathbb{C}\left(\left\|\left(\mathcal{N}_{\perp}+1\right)^{b+2} \boldsymbol{\phi}\right\|_{\mathcal{F}_{\perp}^{\leq N}}^{2}+\left\langle\left(\mathcal{N}_{\perp}+1\right)^{b+2} \phi, \mathrm{d} \Gamma_{\perp}(h)\left(\mathcal{N}_{\perp}+1\right)^{b+2} \phi\right\rangle_{\mathcal{F} \leq N}\right) \\
& \leq \mathbb{C}\left(\left\|\left(\mathcal{N}_{\perp}+1\right)^{b+2} \phi\right\|^{2}\right. \\
&\left.+\left\langle\left(\mathcal{N}_{\perp}+1\right)^{b+2} \phi,\left(\mathbb{H}_{\leq N}+c N^{\frac{1}{3}}\right)\left(\mathcal{N}_{\perp}+1\right)^{b+2} \phi\right\rangle_{\mathcal{F} \leq N}\right) \\
& \leq \mathbb{C}\left(N^{\frac{1}{6}}\left\|\left(\mathcal{N}_{\perp}+1\right)^{b+2} \phi\right\|\right. \\
&\left.+\left.\left|\left\langle\phi,\left(\mathcal{N}_{\perp}+1\right)^{b+2} \mathbb{H}_{\leq N}\left(\mathcal{N}_{\perp}+1\right)^{b+2} \phi\right\rangle_{\mathcal{F}}\right|_{\perp N}\right|^{\frac{1}{2}}\right)^{2}
\end{aligned}
$$

In particular, this implies that

$$
\left\|\left(\mathcal{N}_{\perp}+1\right)^{b} \mathbb{K}_{4} \chi^{(n)}\right\|=\left\|\left(\mathcal{N}_{\perp}+1\right)^{b} \mathbb{K}_{4}^{-} \chi^{(n)}\right\| \leq \mathfrak{C}(n, b) N^{\frac{1}{6}}
$$

by part (a) and Lemma 5.2b. To improve this a priori bound, we apply a similar argument to the bootstrapping in part (a). As in equation (5.70),

$$
\begin{aligned}
\operatorname{Tr} & \left.\mathbb{P}^{(n)}\left(\mathcal{N}_{\perp}+1\right)^{2 b} \mathbb{K}_{4}^{2}\right)=\operatorname{Tr}\left(\mathbb{P}^{(n)}\left(\mathcal{N}_{\perp}+1\right)^{2 b}\left(\mathbb{K}_{4}^{-}\right)^{2}\right) \\
= & \operatorname{Tr}\left(\mathbb{P}_{0}^{(n)}\left(\mathcal{N}_{\perp}+1\right)^{2 b}\left(\mathbb{K}_{4}^{-}\right)^{2}\right) \\
& +\lambda_{N}^{\frac{1}{2}} \operatorname{Tr}\left(\frac{1}{2 \pi \mathrm{i}} \oint_{\gamma^{(n)}} \frac{1}{z-E_{0}^{(n)}} \frac{\mathbb{Q}^{(n)}}{z-\mathbb{H}^{<}} \mathbb{R}_{0} \mathbb{P}_{0}^{(n)}\left(\mathcal{N}_{\perp}+1\right)^{2 b}\left(\mathbb{K}_{4}^{-}\right)^{2} \mathrm{~d} z\right) \\
& +\lambda_{N}^{\frac{1}{2}} \operatorname{Tr}\left(\frac{1}{2 \pi \mathrm{i}} \oint_{\gamma^{(n)}} \frac{\mathbb{P}^{(n)}}{z-\mathbb{H}^{<}} \mathbb{R}_{0} \frac{1}{z-\mathbb{H}_{0}}\left(\mathcal{N}_{\perp}+1\right)^{2 b}\left(\mathbb{K}_{4}^{-}\right)^{2} \mathrm{~d} z\right) .
\end{aligned}
$$

Since $\left[\mathbb{K}_{4}^{-}, \mathcal{N}_{\perp}\right]=0$, Lemma 5.2a implies for the first term that

$$
(5.87 a)=\sum_{m=1}^{\delta_{0}^{(n)}}\left\|\mathbb{K}_{4}\left(\mathcal{N}_{\perp}+1\right)^{b} \chi_{0}^{(n, m)}\right\|_{\mathcal{F}_{\perp}^{\leq N}}^{2} \leq \mathfrak{C}(n, b)
$$

In expression (5.87b), this leads - for $z \in \gamma^{(n)}$ and $\chi_{0}^{(n, m)} \in \mathfrak{E}_{0}^{(n)}-$ to

$$
\begin{aligned}
& N^{-\frac{1}{2}}\left|\left\langle\mathbb{K}_{4}^{-}\left(\mathcal{N}_{\perp}+1\right)^{2 b+2} \chi_{0}^{(n, m)}, \mathbb{K}_{4}^{-}\left(\mathcal{N}_{\perp}+1\right)^{-2} \frac{\mathbb{Q}^{(n)}}{z-\mathbb{H} \mathbb{R}^{<}} \mathbb{R}_{0} \chi_{0}^{(n, m)}\right\rangle\right| \\
& \quad \leq \mathbb{C} N^{-\frac{1}{2}}\left\|\left(\mathcal{N}_{\perp}+1\right)^{2 b+4} \chi_{0}^{(n, m)}\right\|
\end{aligned}
$$




$$
\begin{aligned}
& \times\left(N^{\frac{1}{6}}\left\|\frac{\mathbb{Q}^{(n)}}{z-\mathbb{H}^{<}} \mathbb{R}_{0} \chi_{0}^{(n, m)}\right\|+\left|\left\langle\frac{\mathbb{Q}^{(n)}}{z-\mathbb{H}<} \mathbb{R}_{0} \chi_{0}^{(n, m)}, \mathbb{H}^{<} \frac{\mathbb{Q}^{(n)}}{z-\mathbb{H}^{<}} \mathbb{R}_{0} \chi_{0}^{(n, m)}\right\rangle\right|^{\frac{1}{2}}\right) \\
\leq & \mathbb{C}(n, b) N^{-\frac{1}{3}},
\end{aligned}
$$

where we use Lemmas 5.2a and 5.2b for the left-hand side and equation (5.85) for the right-hand side of the inner product in the first line, as well as Lemmas 5.5a and 5.3b. Finally, for expression (5.87c), equation (5.86) and Lemma 5.2a imply that

$$
\begin{aligned}
& N^{-\frac{1}{2}} \|\left\langle\chi^{(n, m)}, \mathbb{R}_{0} \frac{1}{z-\mathbb{H}_{0}}\left(\mathcal{N}_{\perp}+1\right)^{2 b}\left(\mathbb{K}_{4}^{-}\right)^{2} \chi^{(n, m)}\right\rangle \mid \\
& \leq N^{-\frac{1}{2}}\left\|\left(\mathcal{N}_{\perp}+1\right)^{2 b} \mathbb{K}_{4} \chi^{(n, m)}\right\|\left\|\mathbb{K}_{4} \frac{1}{z-\mathbb{H}_{0}} \mathbb{R}_{0} \chi^{(n, m)}\right\| \\
& \leq \mathbb{C}(n, b) N^{-\frac{1}{3}}\left\|\left(\mathcal{N}_{\perp}+1\right)^{\frac{3}{2}}\left(\mathbb{R}_{0}^{(1)}+\lambda_{N}^{\frac{1}{2}} \mathbb{K}_{4}^{-}\right) \chi^{(n, m)}\right\| \leq \mathbb{C}(n, b) N^{-\frac{1}{3}}
\end{aligned}
$$

by definition (3.46a) of $\mathbb{R}_{0}$ and by part (a). In summary, we find

$$
\operatorname{Tr} \mathbb{P}^{(n)}\left(\mathcal{N}_{\perp}+1\right)^{2 b} \mathbb{K}_{4}^{2}=\sum_{m=1}^{\delta_{0}^{(n)}}\left\|\left(\mathcal{N}_{\perp}+1\right)^{b} \mathbb{K}_{4} \chi^{(n, m)}\right\|^{2} \leq \mathfrak{C}(n, b)
$$

\subsection{Proof of the main results}

In the following, we consider

$$
\mathbb{A} \in\left\{\mathbb{A}_{\text {red }}^{(m)}, \mathbb{1}\right\}
$$

for $j \in \mathbb{N}_{0}$. By Lemma 5.4, $\mathbb{A}$ satisfies

$$
\|\mathbb{A} \phi\| \leq \mathfrak{C} N^{\alpha}\left(\left\|\left(\mathcal{N}_{\perp}+1\right) \phi\right\|+\left\|\mathbb{H}_{0} \phi\right\|\right)
$$

for

$$
\alpha= \begin{cases}-\frac{1}{2} & \text { if } \mathbb{A}=\mathbb{A}_{\text {red }}^{(m)} \\ 0 & \text { if } \mathbb{A}=\mathbb{1}\end{cases}
$$

\subsubsection{Proof of Theorem 1}

Recall that by Proposition 3.14,

$$
\operatorname{Tr} \mathbb{A P}^{(n)}=\sum_{\ell=0}^{a} \lambda_{N}^{\frac{\ell}{2}} \operatorname{Tr} \mathbb{P}_{\ell}^{(n)}+\lambda_{N}^{\frac{a+1}{2}}\left(\operatorname{Tr} \mathbb{A B}_{P}^{(n)}(a)+\operatorname{Tr} \mathbb{B}_{Q}^{(n)}(a)\right)
$$

where

$$
\begin{aligned}
& \mathbb{B}_{P}^{(n)}(a) \\
& \quad=\sum_{\nu=0}^{a} \sum_{m=1}^{a-v} \sum_{j \in \mathbb{N}^{m}} \frac{1}{2 \pi \mathrm{i}} \oint_{\gamma^{(n)}} \frac{\mathbb{P}^{(n)}}{z-\mathbb{H}^{<}} \mathbb{R}_{v} \frac{1}{z-\mathbb{H}_{0}} \mathbb{H}_{j_{1}} \frac{1}{z-\mathbb{H}_{0}} \cdots \mathbb{H}_{j_{m}} \frac{1}{z-\mathbb{H}_{0}} \mathrm{~d} z
\end{aligned}
$$


and

$$
\begin{aligned}
& \mathbb{B}_{Q}^{(n)}(a) \\
& \quad=\sum_{\nu=0}^{a} \sum_{m=1}^{a-v} \sum_{\substack{\boldsymbol{j} \in \mathbb{N}^{m} \\
|\boldsymbol{j}|=a-v}} \sum_{\ell=0}^{m} \sum_{\substack{\boldsymbol{k} \in\{0,1\}^{m+1} \\
|\boldsymbol{k}|=\ell}} \frac{1}{2 \pi \mathrm{i}} \oint_{\gamma^{(n)}} \frac{\mathbb{Q}^{(n)}}{z-\mathbb{H}^{<}} \mathbb{R}_{v} \frac{\mathbb{I}_{k_{1}}^{(n)}}{z-\mathbb{H}_{0}} \mathbb{H}_{j_{1}} \cdots \mathbb{H}_{j_{m}} \frac{\mathbb{I}_{k_{m+1}}^{(n)}}{z-\mathbb{H}_{0}} \mathrm{~d} z
\end{aligned}
$$

with $\mathbb{I}_{0}^{(n)}=\mathbb{P}_{0}^{(n)}$ and $\mathbb{I}_{1}^{(n)}=\mathbb{Q}_{0}^{(n)}$.

Estimates for $\mathbb{B}_{P}^{(n)}(a)$.

Let $\left\{\chi^{(n, \ell)}\right\}_{\ell=1}^{\delta_{0}^{(n)}}$ denote an orthonormal basis of $\mathfrak{E}^{(n)}$ such that $\mathbb{H} \chi^{(n, \ell)}=E^{(n, \ell)} \chi^{(n, \ell)}$. Consequently, $\mathbb{P}^{(n)}=\sum_{\ell=1}^{\delta_{0}^{(n)}}\left|\chi^{(n, \ell)}\right\rangle\left\langle\chi^{(n, \ell)}\right|$, and interchanging trace and contour integral by Fubini's theorem yields

$$
\begin{aligned}
\left|\operatorname{Tr} \mathbb{A}_{P}^{(n)}(a)\right| \leq & \mathbb{C} \sum_{\nu=0}^{a} \sum_{m=1}^{a-v} \sum_{\substack{\boldsymbol{j} \in \mathbb{N}^{m} \\
|\boldsymbol{j}|=a-\nu}} \sum_{\ell=1}^{\delta_{0}^{(n)}} \oint_{\gamma^{(n)}}\left|\frac{1}{z-E^{(n, \ell)}}\right| \\
& \times \mid\left\langle\chi^{(n, \ell)}, \mathbb{R}_{\nu} \frac{1}{z-\mathbb{H}_{0}} \mathbb{H}_{j_{1}} \cdots \mathbb{H}_{j_{m}} \frac{1}{z-\mathbb{H}_{0}} \mathbb{A}^{(n, \ell)}\right\rangle \mathrm{d} z .
\end{aligned}
$$

Lemmas $5.3 \mathrm{a}$ and $5.5 \mathrm{~b}$ lead to the estimate

$$
\left\|\left(\mathcal{N}_{\perp}+1\right)^{b} \mathbb{H}_{j} \frac{\mathbb{I}^{(n)}}{z-\mathbb{H}_{0}} \boldsymbol{\phi}\right\| \leq \mathfrak{C}(b, j)\left\|\left(\mathcal{N}_{\perp}+1\right)^{b+\frac{j}{2}+1} \phi\right\|
$$

for $\mathbb{I}^{(n)} \in\left\{\mathbb{1}, \mathbb{P}_{0}^{(n)}, \mathbb{Q}_{0}^{(n)}\right\}$, hence

$$
\begin{aligned}
& \|\left\langle\chi^{(n, \ell)}, \mathbb{R}_{v} \frac{1}{z-\mathbb{H}_{0}} \mathbb{H}_{j_{1}} \frac{1}{z-\mathbb{H}_{0}} \cdots \mathbb{H}_{j_{m}} \frac{1}{z-\mathbb{H}_{0}} \mathbb{A}^{(n, \ell)}\right\rangle \mid \\
& \quad \leq\left\|\chi^{(n, \ell)}\right\|\left\|\mathbb{A} \frac{1}{z-\mathbb{H}_{0}} \mathbb{H}_{j_{m}} \frac{1}{z-\mathbb{H}_{0}} \cdots \mathbb{H}_{j_{1}} \frac{1}{z-\mathbb{H}_{0}} \mathbb{R}_{v} \chi^{(n, \ell)}\right\| \\
& \leq \mathbb{C} N^{\alpha}\left\|\mathbb{H}_{j_{m}} \frac{1}{z-\mathbb{H}_{0}} \cdots \mathbb{H}_{j_{1}} \frac{1}{z-\mathbb{H}_{0}} \mathbb{R}_{v} \chi^{(n, \ell)}\right\| \\
& \quad \leq \mathbb{C}(n, a) N^{\alpha}\left\|\left(\mathcal{N}_{\perp}+1\right)^{\frac{3}{2}(a-v)} \mathbb{R}_{v} \chi^{(n, m)}\right\| \leq \mathbb{C}(n, a) N^{\alpha}
\end{aligned}
$$

by Lemmas 5.4, 5.3b and 5.6. Here we use the facts that $\mathbb{R}_{0}=\mathbb{R}_{0}^{(1)}+\lambda_{N}^{\frac{1}{2}} \mathbb{K}_{4}$ and $\mathbb{R}_{1}=\mathbb{R}_{1}^{(1)}+\mathbb{K}_{4}$, and apply Lemmas 5.2a and 5.6. In summary, this yields

$$
\left|\operatorname{Tr} \mathbb{B}_{P}^{(n)}(a)\right| \leq N^{\alpha} \mathfrak{C}(n, a)
$$


Estimates for $\mathbb{B}_{Q}^{(n)}(a)$.

By the definition of $\mathbb{B}_{Q}^{(n)}$, it follows that

$$
\begin{aligned}
&\left|\operatorname{Tr} \mathbb{B}_{Q}^{(n)}(a)\right| \leq \mathbb{C}(n) \sum_{\nu=0}^{a} \sum_{m=1}^{a-v} \sum_{\substack{\boldsymbol{j} \in \mathbb{N}^{m} \\
|\boldsymbol{j}|=a-v}} \sum_{\ell=0}^{m} \sum_{\substack{\boldsymbol{k} \in\{0,1\}^{m+1} \\
|\boldsymbol{k}|=\ell}} \\
& \sup _{z \in \gamma^{(n)}}\left|\operatorname{Tr} \mathbb{A} \frac{\mathbb{Q}^{(n)}}{z-\mathbb{H}^{<}} \mathbb{R}_{v} \frac{\mathbb{I}_{k_{1}}^{(n)}}{z-\mathbb{H}_{0}} \mathbb{H}_{j_{1}} \frac{\mathbb{I}_{k_{2}}^{(n)}}{z-\mathbb{H}_{0}} \cdots \mathbb{H}_{j_{m}} \frac{\mathbb{I}_{k_{m+1}}^{(n)}}{z-\mathbb{H}_{0}}\right| .
\end{aligned}
$$

Each term contains at least one projector $\mathbb{P}_{0}^{(n)}$-that is, there exists some $\sigma \in\{1, \ldots, m+1\}$ such that $k_{\sigma}=0$. Decomposing $\mathbb{P}_{0}^{(n)}=\sum_{\mu=1}^{\delta_{0}^{(n)}}\left|\chi_{0}^{(n, \mu)}\right\rangle\left\langle\chi_{0}^{(n, \mu)}\right|$ for a basis $\left\{\chi_{0}^{(n, \mu)}\right\}_{\mu=1}^{\delta_{0}^{(n)}}$ of $\mathfrak{E}_{0}^{(n)}$ as in Lemma 4.7c, we obtain

$$
\begin{aligned}
& \left|\operatorname{Tr} \mathbb{A} \frac{\mathbb{Q}^{(n)}}{z-\mathbb{H}^{<}} \mathbb{R}_{v} \frac{\mathbb{I}_{k_{1}}^{(n)}}{z-\mathbb{H}_{0}} \mathbb{H}_{j_{1}} \frac{\mathbb{I}_{k_{2}}^{(n)}}{z-\mathbb{H}_{0}} \cdots \mathbb{H}_{j_{m}} \frac{\mathbb{I}_{k_{m+1}}^{(n)}}{z-\mathbb{H}_{0}}\right| \\
& \leq \mathbb{C}(n) \sum_{\mu=1}^{\delta_{0}^{(n)}}\left\|\frac{\mathbb{Q}^{(n)}}{z-\mathbb{H}^{<}} \mathbb{R}_{v} \frac{\mathbb{I}_{k_{1}}^{(n)}}{z-\mathbb{H}_{0}} \mathbb{H}_{j_{1}} \cdots \frac{\mathbb{I}_{k_{\sigma-1}}^{(n)}}{z-\mathbb{H}_{0}} \mathbb{H}_{j_{\sigma-1}} \chi_{0}^{(n, \mu)}\right\| \\
& \quad \times\left\|\mathbb{A} \frac{\mathbb{I}_{k_{m+1}}^{(n)}}{z-\mathbb{H}_{0}} \mathbb{H}_{j_{m}} \frac{\mathbb{I}_{k_{m}}^{(n)}}{z-\mathbb{H}_{0}} \cdots \frac{\mathbb{I}_{k_{\sigma+1}}^{(n)}}{z-\mathbb{H}_{0}} \mathbb{H}_{j_{\sigma}} \chi_{0}^{(n, \mu)}\right\|
\end{aligned}
$$

Using estimate (5.94) in combination with Lemmas 5.3 and 5.2b, we find for expression (5.98a)

$$
\begin{aligned}
& \left\|\frac{\mathbb{Q}^{(n)}}{z-\mathbb{H}^{<}} \mathbb{R}_{v} \frac{\mathbb{I}_{k_{1}}^{(n)}}{z-\mathbb{H}_{0}} \mathbb{H}_{j_{1}} \cdots \frac{\mathbb{I}_{k_{\sigma-1}}^{(n)}}{z-\mathbb{H}_{0}} \mathbb{H}_{j_{\sigma-1}} \chi_{0}^{(n, \mu)}\right\| \\
& \quad \leq \mathbb{C}(n, a)\left\|\left(\mathcal{N}_{\perp}+1\right)^{\frac{v+2 \sigma+1+j_{1}+\cdots+j_{\sigma-1}}{2}} \chi_{0}^{(n, \mu)}\right\|
\end{aligned}
$$

analogously to before, and for expression $(5.98 b)$

$$
\begin{aligned}
& \left\|\mathbb{A} \frac{\mathbb{I}_{k_{m+1}}^{(n)}}{z-\mathbb{H}_{0}} \mathbb{H}_{j_{m}} \frac{\mathbb{I}_{k_{m}}^{(n)}}{z-\mathbb{H}_{0}} \cdots \frac{\mathbb{I}_{k_{\sigma+1}}^{(n)}}{z-\mathbb{H}_{0}} \mathbb{H}_{j_{\sigma}} \chi_{0}^{(n, \mu)}\right\| \\
& \quad \leq N^{\alpha} \mathfrak{C}(n, a)\left\|\left(\mathcal{N}_{\perp}+1\right)^{\frac{j_{\sigma+1}+\cdots+j_{m}+2(m-\sigma)}{2}} \mathbb{H}_{j_{\sigma}} \chi_{0}^{(n, \mu)}\right\| \\
& \leq N^{\alpha} \mathfrak{C}(n, a)\left\|\left(\mathcal{N}_{\perp}+1\right)^{\frac{j_{\sigma}+\cdots+j_{m}+2(m-\sigma+1)}{2}} \chi_{0}^{(n, \mu)}\right\|,
\end{aligned}
$$

since $\mathbb{H}_{0} \chi_{0}^{(n, \mu)}=E_{0}^{(n)} \chi_{0}^{(n, \mu)}$. Combining both estimates yields, with Lemma $4.7 \mathrm{~d}$,

$$
\left|\operatorname{Tr} \mathbb{B}_{Q}^{(n)}(a)\right| \leq \mathfrak{C}(n, a) N^{\alpha}
$$




\subsubsection{Proof of Corollary 3.4}

For any bounded operator $\mathbb{A} \in \mathcal{L}\left(\mathcal{F}_{\perp}\right)$, Proposition 3.14 implies that

$$
\left|\operatorname{Tr} \mathbb{P}^{(n)}-\sum_{\ell=0}^{a} \lambda_{N}^{\frac{\ell}{2}} \operatorname{Tr} \mathbb{A P}_{\ell}^{(n)}\right| \leq \lambda_{N}^{\frac{a+1}{2}}\left(\left|\operatorname{Tr} \mathbb{A B}_{P}^{(n)}(a)\right|+\left|\operatorname{Tr} \mathbb{A B}_{Q}^{(n)}(a)\right|\right),
$$

and one infers from the previous section that

$$
\left|\operatorname{Tr} \mathbb{B}_{P}^{(n)}(a)\right|+\left|\operatorname{Tr} \mathbb{B}_{Q}^{(n)}(a)\right| \leq\|\mathbb{A}\|_{\text {op }} \mathfrak{C}(n, a) .
$$

Consequently,

$$
\operatorname{Tr}\left|\mathbb{P}^{(n)}-\sum_{\ell=0}^{a} \lambda_{N}^{\frac{\ell}{2}} \mathbb{P}_{\ell}^{(n)}\right|=\sup _{\substack{\mathbb{A} \text { compact } \\\|\mathbb{A}\|_{\text {op }}=1}}\left|\operatorname{Tr} \mathbb{P}^{(n)}-\sum_{\ell=0}^{a} \lambda_{N}^{\frac{\ell}{2}} \operatorname{Tr} \mathbb{A P}_{\ell}^{(n)}\right| \leq \lambda_{N}^{\frac{a+1}{2}} \mathfrak{C}(n, a) .
$$

\subsubsection{Proof of Theorem 2}

Let us abbreviate $\oint_{\gamma^{(n)}}^{\prime}:=\frac{1}{2 \pi \mathrm{i}} \oint_{\gamma^{(n)}}$. Note first that

$$
\begin{aligned}
\sum_{\nu \in \iota^{(n)}} \delta_{N}^{(v)} E^{(v)} & =\operatorname{Tr} \mathbb{H} \mathbb{P}^{(n)}=\operatorname{Tr} \oint_{\gamma^{(n)}}^{\prime} \frac{\mathbb{H}}{z-\mathbb{H}} \mathrm{d} z=\operatorname{Tr} \oint_{\gamma^{(n)}}^{\prime} \frac{z}{z-\mathbb{H}} \mathrm{d} z \\
& =E_{0}^{(n)} \operatorname{Tr} \mathbb{P}^{(n)}+\operatorname{Tr} \oint_{\gamma^{(n)}}^{\prime} \frac{z-E_{0}^{(n)}}{z-\mathbb{H}} \mathrm{d} z .
\end{aligned}
$$

Since $\operatorname{Tr} \mathbb{P}^{(n)}=\delta_{0}^{(n)}$ and

$$
\oint_{\gamma^{(n)}}^{\prime} \frac{z-E_{0}^{(n)}}{z-\mathbb{H}_{0}} \mathrm{~d} z=\mathbb{P}_{0}^{(n)} \oint_{\gamma^{(n)}}^{\prime} 1 \mathrm{~d} z+\oint_{\gamma^{(n)}}^{\prime} \frac{\mathbb{Q}_{0}^{(n)}}{z-\mathbb{H}_{0}}\left(z-E_{0}^{(n)}\right) \mathrm{d} z=0,
$$

this implies by Lemma 3.13 that

$$
\begin{aligned}
\operatorname{Tr} \mathbb{H} \mathbb{P}^{(n)}= & \delta_{0}^{(n)} E_{0}^{(n)}+\sum_{\ell=1}^{a} \lambda_{N}^{\frac{\ell}{2}} \sum_{\nu=1}^{\ell} \sum_{\substack{\boldsymbol{j} \in \mathbb{N}^{\nu} \\
|\boldsymbol{j}|=\ell}} \operatorname{Tr} \oint_{\gamma^{(n)}}^{\prime} \frac{1}{z-\mathbb{H}_{0}} \mathbb{H}_{j_{1}} \frac{1}{z-\mathbb{H}_{0}} \cdots \mathbb{H}_{j_{v}} \frac{z-E_{0}^{(n)}}{z-\mathbb{H}_{0}} \mathrm{~d} z \\
& +\lambda_{N}^{\frac{a+1}{2}} \sum_{\nu=0}^{a} \sum_{m=1}^{a-v} \sum_{\substack{\boldsymbol{j} \in \mathbb{N}^{m} \\
|\boldsymbol{j}|=a-\nu}} \operatorname{Tr} \oint_{\gamma^{(n)}}^{\prime} \frac{1}{z-\mathbb{H}^{<}} \mathbb{R}_{v} \frac{1}{z-\mathbb{H}_{0}} \mathbb{H}_{j_{1}} \frac{1}{z-\mathbb{H}_{0}} \cdots \mathbb{H}_{j_{m}} \frac{z-E_{0}^{(n)}}{z-\mathbb{H}_{0}} \mathrm{~d} z .
\end{aligned}
$$

For $z \in \gamma^{(n)}$, it holds that $\left|z-E_{0}^{(n)}\right| \leq \mathfrak{C}$; hence the proof of Theorem 1 for $\mathbb{A}=\mathbb{1}$ yields

$$
|(5.106 b)| \leq \lambda_{N}^{\frac{a+1}{2}} \mathfrak{C}(n, a) .
$$

Moreover, all half-integer powers of $\lambda_{N}$ in expression (5.106a) vanish by parity: define the unitary map

$$
\mathcal{U}_{P}: \mathcal{F} \rightarrow \mathcal{F}, \quad \mathcal{U}_{P} a^{\dagger}(f) \mathcal{U}_{P}=a^{\dagger}(-f)=-a^{\dagger}(f),
$$

for any $f \in \mathfrak{H}$. Clearly, $\mathcal{U}_{P}$ preserves $\mathcal{F}_{\perp}$ and acts on the operator-valued distributions $a_{x}^{\dagger}$ and $a_{x}$ as $\mathcal{U}_{P} a_{x}^{\dagger} \mathcal{U}_{P}=-a_{x}^{\dagger}$ and $\mathcal{U}_{P} a_{x} \mathcal{U}_{P}=-a_{x}$. By definition (3.9), $\mathbb{H}_{j}$ contains an even number of 
creation/annihilation operators for $j$ even and an odd number for $j$ odd, hence

$$
\mathcal{U}_{P} \mathbb{H}_{j} \mathcal{U}_{P}=(-1)^{j} \mathbb{H}_{j}, \quad \mathcal{U}_{P} \frac{1}{z-\mathbb{H}_{0}} \mathcal{U}_{P}=\frac{1}{z-\mathbb{H}_{0}},
$$

because $\mathcal{U}_{P} \mathbb{H}_{0}^{\ell} \mathcal{U}_{P}=\mathbb{H}_{0}^{\ell}$ for any $\ell \in \mathbb{R}$. Consequently,

$$
\begin{aligned}
\operatorname{Tr} \frac{1}{z-\mathbb{H}_{0}} \mathbb{H}_{j_{1}} \cdots \mathbb{H}_{j_{v}} \frac{1}{z-\mathbb{H}_{0}} & =\operatorname{Tr} \mathcal{U}_{P} \frac{1}{z-\mathbb{H}_{0}} \mathbb{H}_{j_{1}} \cdots \mathbb{H}_{j_{v}} \frac{1}{z-\mathbb{H}_{0}} \mathcal{U}_{P} \\
& =(-1)^{\ell} \operatorname{Tr} \frac{1}{z-\mathbb{H}_{0}} \mathbb{H}_{j_{1}} \cdots \mathbb{H}_{j_{v}} \frac{1}{z-\mathbb{H}_{0}}
\end{aligned}
$$

for any $\boldsymbol{j}$ such that $|\boldsymbol{j}|=\ell$. This yields

$$
\operatorname{Tr} H \mathbb{P}^{(n)}=\delta_{0}^{(n)} E_{0}^{(n)}+\sum_{\ell=1}^{a} \lambda_{N}^{\ell} \sum_{\nu=1}^{2 \ell} \mathbb{E}_{\ell, v}^{(n)}+\mathcal{O}\left(\lambda_{N}^{a+1}\right)
$$

with

$$
\mathbb{E}_{\ell, v}^{(n)}:=\sum_{\substack{\boldsymbol{j} \in \mathbb{N}^{v} \\|\boldsymbol{j}|=2 \ell}} \oint_{\gamma^{(n)}}^{\prime} \operatorname{Tr}\left(\frac{1}{z-\mathbb{H}_{0}}\right)^{2} \mathbb{H}_{j_{1}} \frac{1}{z-\mathbb{H}_{0}} \cdots \mathbb{H}_{j_{v}}\left(z-E_{0}^{(n)}\right) \mathrm{d} z
$$

For $v=1$, one computes

$$
\mathbb{E}_{\ell, 1}^{(n)}=\oint_{\gamma^{(n)}}^{\prime} \operatorname{Tr}_{0}^{(n)} \mathbb{H}_{2 \ell} \frac{\mathrm{d} z}{z-E_{0}^{(n)}}=\operatorname{Tr}_{0}^{(n)} \mathbb{H}_{2 \ell}
$$

For $v \geq 2$, we decompose each identity as $1=\mathbb{P}_{0}^{(n)}+\mathbb{Q}_{0}^{(n)}$ and order the summands according to the number $k$ of projections $\mathbb{Q}_{0}^{(n)}$, which yields

$$
\mathbb{E}_{\ell, v}^{(n)}=\sum_{k=1}^{v-2} \mathbb{E}_{\ell, v, k}^{(n)}+\mathbb{E}_{\ell, v, v-1}^{(n)}
$$

with

$$
\mathbb{E}_{\ell, v, v-1}^{(n)}=\sum_{\substack{\boldsymbol{j} \in \mathbb{N}^{v} \\|\boldsymbol{j}|=2 \ell}} \operatorname{Tr} \mathbb{P}_{0}^{(n)} \mathbb{H}_{j_{1}} \mathbb{O}_{1}^{(n)} \cdots \mathbb{O}_{1}^{(n)} \mathbb{H}_{j_{v}}
$$

for $\mathbb{O}_{m}^{(n)}$ as in Definition 3.3, and

$$
\begin{aligned}
& \mathbb{E}_{\ell, v, k}^{(n)}=\sum_{\substack{\boldsymbol{j} \in \mathbb{N}^{v} \\
|\boldsymbol{j}|=2 \ell}}\left(\oint_{\gamma^{(n)}}^{\prime} \operatorname{Tr}_{0}^{(n)}\left[\mathbb{H}_{j_{1}} \widetilde{\mathbb{O}}_{1}^{(n)} \cdots \mathbb{H}_{j_{k}} \widetilde{\mathbb{O}}_{1}^{(n)} \mathbb{H}_{j_{k+1}} \mathbb{P}_{0}^{(n)} \cdots \mathbb{P}_{0}^{(n)} \mathbb{H}_{j_{v}}\right]_{\mathrm{p}}\right. \\
& \times \frac{\mathrm{d} z}{\left(z-E_{0}^{(n)}\right)^{\nu-k}} \\
&+\oint_{\gamma^{(n)}}^{\prime} \operatorname{Tr} \widetilde{\mathbb{O}}_{2}^{(n)}\left[\mathbb{H}_{j_{1}} \widetilde{\mathbb{O}}_{1}^{(n)} \cdots \mathbb{H}_{j_{k-1}} \widetilde{\mathbb{O}}_{1}^{(n)} \mathbb{H}_{j_{k}} \mathbb{P}_{0}^{(n)} \cdots \mathbb{P}_{0}^{(n)} \mathbb{H}_{j_{\nu}}\right]_{\mathrm{p}}
\end{aligned}
$$




$$
\left.\times \frac{\mathrm{d} z}{\left(z-E_{0}^{(n)}\right)^{v-k-1}}\right)
$$

for $k \leq v-2$. Here, we abbreviate

$$
\widetilde{\mathbb{O}}_{m}^{(n)}=\frac{\mathbb{Q}_{0}^{(n)}}{\left(z-\mathbb{H}_{0}\right)^{m}}
$$

and the notation $[\cdot]_{\mathrm{p}}$ indicates the sum of all possibilities to distribute the operators $\mathbb{P}_{0}^{(n)}$ over the slots between the operators $\mathbb{H}_{j}$. By cyclicity of the trace,

$$
\begin{aligned}
& \sum_{\substack{\boldsymbol{j} \in \mathbb{N}^{\nu} \\
|\boldsymbol{j}|=2 \ell}} \operatorname{Tr} \mathbb{P}_{0}^{(n)}\left[\mathbb{H}_{j_{1}} \widetilde{\mathbb{O}}_{1}^{(n)} \cdots \mathbb{H}_{j_{k}} \widetilde{\mathbb{O}}_{1}^{(n)} \mathbb{H}_{j_{k+1}} \mathbb{P}_{0}^{(n)} \cdots \mathbb{P}_{0}^{(n)} \mathbb{H}_{j_{v}}\right]_{\mathrm{p}} \\
& \quad=\sum_{\substack{\boldsymbol{j} \in \mathbb{N}^{v} \\
|\boldsymbol{j}|=2 \ell}} \frac{v-k}{v} \operatorname{Tr}\left[\widetilde{\mathbb{O}}_{1}^{(n)} \mathbb{H}_{j_{1}} \cdots \widetilde{\mathbb{O}}_{1}^{(n)} \mathbb{H}_{j_{k}} \mathbb{P}_{0}^{(n)} \mathbb{H}_{j_{k+1}} \cdots \mathbb{P}_{0}^{(n)} \mathbb{H}_{j_{v}}\right]_{\mathrm{p}}
\end{aligned}
$$

which can be seen by observing that the first line is a sum of $\left(\begin{array}{c}v-1 \\ k\end{array}\right)$ terms while the sum in the second line has $\left(\begin{array}{c}v \\ k\end{array}\right)=\frac{v}{v-k}\left(\begin{array}{c}v-1 \\ k\end{array}\right)$ addends. Next we note that for any $f$ which is holomorphic in the interior of $\gamma^{(n)}$, the residue theorem implies that

$$
\oint_{\gamma^{(n)}}^{\prime} f(z) \frac{\mathrm{d} z}{\left(z-E_{0}^{(n)}\right)^{v-k}}=\frac{1}{v-k-1} \oint_{\gamma^{(n)}}^{\prime} f^{\prime}(z) \frac{\mathrm{d} z}{\left(z-E_{0}^{(n)}\right)^{v-k-1}} .
$$

Since

$$
\frac{\mathrm{d}^{m}}{\mathrm{~d} z^{m}} \widetilde{\mathbb{O}}_{1}^{(n)}=(-1)^{m} m ! \widetilde{\mathbb{O}}_{m+1}^{(n)},
$$

it follows that

$$
\begin{aligned}
& \sum_{\substack{\boldsymbol{j} \in \mathbb{N}^{v} \\
|\boldsymbol{j}|=2 \ell}} \frac{\mathrm{d}}{\mathrm{d} z} \operatorname{Tr}\left[\widetilde{\mathbb{O}}_{1}^{(n)} \mathbb{H}_{j_{1}} \cdots \widetilde{\mathbb{O}}_{1}^{(n)} \mathbb{H}_{j_{k}} \mathbb{P}_{0}^{(n)} \mathbb{H}_{j_{k+1}} \cdots \mathbb{P}_{0}^{(n)} \mathbb{H}_{j_{v}}\right]_{\mathrm{p}} \\
& \quad=-v \sum_{\substack{\boldsymbol{j} \in \mathbb{N}^{v} \\
|\boldsymbol{j}|=2 \ell}} \operatorname{Tr} \widetilde{\mathbb{O}}_{2}^{(n)}\left[\mathbb{H}_{j_{1}} \widetilde{\mathbb{O}}_{1}^{(n)} \cdots \widetilde{\mathbb{O}}_{1}^{(n)} \mathbb{H}_{j_{k}} \mathbb{P}_{0}^{(n)} \mathbb{H}_{j_{k+1}} \cdots \mathbb{P}_{0}^{(n)} \mathbb{H}_{j_{v}}\right]_{\mathrm{p}}
\end{aligned}
$$

because by the product rule, the first line is a sum of $k\left(\begin{array}{l}v \\ k\end{array}\right)=v\left(\begin{array}{c}v-1 \\ k-1\end{array}\right)$ terms. Integrating by parts yields

$$
\begin{aligned}
& \mathbb{E}_{\ell, v, k}^{(n)} \\
& =\sum_{\substack{\boldsymbol{j} \in \mathbb{N}^{v} \\
|\boldsymbol{j}|=2 \ell}} \frac{1}{v} \oint_{\gamma^{(n)}}^{\prime} \operatorname{Tr}\left[\widetilde{\mathbb{O}}_{1}^{(n)} \mathbb{H}_{j_{1}} \widetilde{\mathbb{O}}_{1}^{(n)} \cdots \widetilde{\mathbb{O}}_{1}^{(n)} \mathbb{H}_{j_{k}} \mathbb{P}_{0}^{(n)} \cdots \mathbb{P}_{0}^{(n)} \mathbb{H}_{j_{v}}\right]_{\mathrm{p}} \frac{\mathrm{d} z}{\left(z-E_{0}^{(n)}\right)^{v-k}} \\
& =\sum_{\substack{\boldsymbol{j} \in \mathbb{N}^{v} \\
j \mid=2 \ell}} \frac{1}{v-k} \oint_{\gamma^{(n)}}^{\prime} \operatorname{Tr} \mathbb{P}_{0}^{(n)}\left[\mathbb{H} \widetilde{\mathbb{O}}_{1}^{(n)} \cdots \mathbb{H}_{j_{k}} \widetilde{\mathbb{O}}_{1}^{(n)} \mathbb{H}_{j_{k+1}} \mathbb{P}_{0}^{(n)} \cdots \mathbb{P}_{0}^{(n)} \mathbb{H}_{j_{v}}\right]_{\mathrm{p}} \\
& \times \frac{\mathrm{d} z}{\left(z-E^{(n)}\right)^{v-k}} .
\end{aligned}
$$


Consequently, the residue theorem and equation (5.119) lead to

$$
\begin{aligned}
& \mathbb{E}_{\ell, v, k}^{(n)} \\
& \quad=\left.\sum_{\substack{\boldsymbol{j} \in \mathbb{N}^{v} \\
|\boldsymbol{j}|=2 \ell}} \frac{1}{(v-k) !} \frac{\mathrm{d}^{v-k-1} \mathrm{~d} z^{v-k-1}}{\operatorname{Tr} \mathbb{P}_{0}^{(n)}}\left[\mathbb{H}_{j_{1}} \widetilde{\mathbb{O}}_{1}^{(n)} \cdots \widetilde{\mathbb{O}}_{1}^{(n)} \mathbb{H}_{j_{k+1}} \mathbb{P}_{0}^{(n)} \cdots \mathbb{P}_{0}^{(n)} \mathbb{H}_{j_{v}}\right]_{\mathrm{p}}\right|_{z=E_{0}^{(n)}} \\
& \quad=\sum_{\substack{\boldsymbol{j} \in \mathbb{N}^{v} \\
|\boldsymbol{j}|=2 \ell}} \frac{(-1)^{v-k-1}}{v-k} \sum_{\substack{\boldsymbol{m} \in \mathbb{N}_{0}^{k} \\
|\boldsymbol{m}|=v-k-1}} \operatorname{Tr}_{0}^{(n)}\left[\mathbb{H}_{j_{1}} \mathbb{O}_{m_{1}+1}^{(n)} \cdots \mathbb{O}_{m_{k}+1}^{(n)} \mathbb{H}_{j_{k+1}} \mathbb{P}_{0}^{(n)} \cdots \mathbb{P}_{0}^{(n)} \mathbb{H}_{j_{v}}\right]_{\mathrm{p}} \\
& \quad=\sum_{\substack{\boldsymbol{j} \in \mathbb{N}^{v} \\
|\boldsymbol{j}|=2 \ell}} \frac{(-1)^{v-k-1}}{v-k} \sum_{\substack{\boldsymbol{m} \in \mathbb{N}^{k} \\
|\boldsymbol{m}|=v-1}} \operatorname{Tr} \mathbb{P}_{0}^{(n)}\left[\mathbb{H}_{j_{1}} \mathbb{O}_{m_{1}}^{(n)} \cdots \mathbb{O}_{m_{k}}^{(n)} \mathbb{H}_{j_{k+1}} \mathbb{P}_{0}^{(n)} \cdots \mathbb{P}_{0}^{(n)} \mathbb{H}_{j_{v}}\right]_{\mathrm{p}}
\end{aligned}
$$

Recall that the subscript 'p' indicates the sum over all possibilities to distribute $\mathbb{P}_{0}$. In particular, this implies that all empty slots are subsequently filled up with the tuple $\left(\mathbb{O}_{m_{1}}^{(n)}, \ldots, \mathbb{O}_{m_{k}}^{(n)}\right)$ without permuting the positions of the $\mathbb{O}_{m_{j}}^{(n)}$. Using the notation $\mathbb{O}_{0}^{(n)}=-\mathbb{P}_{0}^{(n)}$, one can equivalently write

$$
\begin{aligned}
& \sum_{k=1}^{v-1} \mathbb{E}_{\ell, v, k}^{(n)} \\
& \quad=\sum_{\substack{\boldsymbol{j} \in \mathbb{N}^{v} \\
|\boldsymbol{j}|=2 \ell}} \sum_{k=1}^{v-1} \sum_{\substack{\boldsymbol{m} \in \mathbb{N}^{k} \\
|\boldsymbol{m}|=\nu-1}} \frac{1}{v-k} \operatorname{Tr} \mathbb{P}_{0}^{(n)}\left[\mathbb{H}_{j_{1}} \mathbb{O}_{m_{1}}^{(n)} \cdots \mathbb{H}_{j_{k}} \mathbb{O}_{m_{k}}^{(n)} \mathbb{H}_{j_{k+1}} \mathbb{O}_{0}^{(n)} \cdots \mathbb{O}_{0}^{(n)} \mathbb{H}_{j_{v}}\right]_{\mathrm{p}} \\
& =\sum_{\substack{\boldsymbol{j} \in \mathbb{N}^{v} \\
|\boldsymbol{j}|=2 \ell}} \sum_{\substack{\boldsymbol{m} \in \mathbb{N}_{0}^{v-1} \\
|\boldsymbol{m}|=\nu-1}} \frac{1}{\kappa(\boldsymbol{m})} \operatorname{Tr} \mathbb{P}_{0}^{(n)} \mathbb{H}_{j_{1}} \mathbb{O}_{m_{1}}^{(n)} \cdots \mathbb{H}_{j_{v-1}} \mathbb{O}_{m_{\nu-1}}^{(n)} \mathbb{H}_{j_{v}},
\end{aligned}
$$

where we denote by $\kappa(\boldsymbol{m})-1$ the number of operators $\mathbb{O}_{0}^{(n)}$. Finally, in case of a nondegenerate eigenvalue $E_{0}^{(n)}$, some terms vanish by parity, which leads to the simplified equations (3.24).

\section{Appendix A. Excitation Hamiltonian}

For $h$ and $e_{\mathrm{H}}$ as in Lemma 2.2 and

$$
W\left(x_{1}, x_{2}\right)=v\left(x_{1}-x_{2}\right)-\left(v * \varphi^{2}\right)\left(x_{1}\right)-\left(v * \varphi^{2}\right)\left(x_{2}\right)+\left\langle\varphi, v * \varphi^{2} \varphi\right\rangle,
$$

(as in definition (2.43)), it follows that

$$
H_{N}=N e_{\mathrm{H}}+\sum_{j=1}^{N} h_{j}+\lambda_{N} \sum_{1 \leq i<j \leq N} W\left(x_{i}, x_{j}\right)
$$

We denote by $\left\{\varphi_{n}\right\}_{n \geq 0}, \varphi_{0}=\varphi$, an eigenbasis for $h$ and abbreviate

$$
\begin{aligned}
h_{m n} & :=\left\langle\varphi_{m}, h \varphi_{n}\right\rangle, \\
W_{m n p q} & :=\int \mathrm{d} x \mathrm{~d} y \overline{\varphi_{m}(x) \varphi_{n}(y)} W(x, y) \varphi_{p}(x) \varphi_{q}(y)
\end{aligned}
$$


and $a_{m}^{\sharp}:=a^{\sharp}\left(\varphi_{m}\right)$. Since $h_{m 0}=h_{0 n}=0$ and $h_{m n}=0$ for $m \neq n$, it follows that

$$
\begin{aligned}
H_{N}= & N e_{\mathrm{H}}+\sum_{m, n \geq 0} h_{m n} a_{m}^{\dagger} a_{n}+\frac{\lambda_{N}}{2} \sum_{m, n, p, q \geq 0} W_{m n p q} a_{m}^{\dagger} a_{n}^{\dagger} a_{p} a_{q} \\
= & N e_{\mathrm{H}}+\sum_{m>0} h_{m m} a_{m}^{\dagger} a_{m}+\frac{\lambda_{N}}{2} W_{0000} a_{0}^{\dagger} a_{0}^{\dagger} a_{0} a_{0} \\
& +\left(\lambda_{N} \sum_{m>0} W_{000 m} a_{0}^{\dagger} a_{0}^{\dagger} a_{0} a_{m}+\text { h.c. }\right) \\
& +\frac{\lambda_{N}}{2}\left(\sum_{m, n>0} W_{m 0 n 0} a_{m}^{\dagger} a_{0}^{\dagger} a_{n} a_{0}+\text { h.c. }\right)+\left(\frac{\lambda_{N}}{2} \sum_{m, n>0} W_{m n 00} a_{m}^{\dagger} a_{n}^{\dagger} a_{0} a_{0}+\text { h.c. }\right) \\
& +\frac{\lambda_{N}}{2} \sum_{m, n>0}\left(W_{0 m n 0} a_{0}^{\dagger} a_{m}^{\dagger} a_{n} a_{0}+W_{m 00 n} a_{m}^{\dagger} a_{0}^{\dagger} a_{0} a_{n}\right) \\
& +\left(\lambda_{N} \sum_{m, n, p>0} W_{m n p 0} a_{m}^{\dagger} a_{n}^{\dagger} a_{p} a_{0}+\right.\text { h.c. } \\
& +\frac{\lambda_{N}}{2} \sum_{m, n, p, q>0} W_{m n p q} a_{m}^{\dagger} a_{n}^{\dagger} a_{p} a_{q} .
\end{aligned}
$$

As $W_{0000}=W_{000 m}=W_{m 0 n 0}=0, W_{0 m n 0}=\left\langle\varphi_{m}, K_{1} \varphi_{n}\right\rangle_{\mathfrak{G}}, W_{m n 00}=\left\langle\varphi_{m} \otimes \varphi_{n}, K_{2}\right\rangle_{\mathfrak{G}^{2}}$ and $W_{m n p 0}=$ $\left\langle\varphi_{m} \otimes \varphi_{n}, K_{3} \varphi_{p}\right\rangle_{\mathfrak{H}^{2}}$, equation (2.40) follows from equation (A.4) by the substitution rules (2.37).

\section{Appendix B. Asymptotic expansion of the wave function}

Theorem 4. Let $\mathfrak{H}$ be a Hilbert space, let $\chi \in \mathfrak{H}$ with $\|\chi\|=1$ and define $P:=|\chi\rangle\langle\chi|$. Assume that $P$ admits an asymptotic expansion in the small parameter $\varepsilon>0$ - that is, there exists a family of $\varepsilon$-independent operators $\left\{P_{\ell}\right\}_{\ell \in \mathbb{N}_{0}}$ such that for any $a \in \mathbb{N}_{0}$,

$$
\operatorname{Tr}_{\mathfrak{H}}\left|P-\sum_{\ell=0}^{a} \varepsilon^{\ell} P_{\ell}\right| \leq C(a) \varepsilon^{a+1}
$$

for some constant $C(a)>0$ and sufficiently small $\varepsilon$. Moreover, assume that there exists some normalised $\chi_{0} \in \mathfrak{H}$ such that $P_{0}=\left|\chi_{0}\right\rangle\left\langle\chi_{0}\right|$. Then for a suitable choice of the phase of $\chi_{0}$, there exists for any $a \in \mathbb{N}_{0}$ a constant $\widetilde{C}(a)>0$ such that

$$
\left\|\chi-\sum_{\ell=0}^{a} \varepsilon^{\ell} \chi_{\ell}\right\| \leq \widetilde{C}(a) \varepsilon^{a+1},
$$

where

$$
\begin{aligned}
\chi_{\ell} & :=\sum_{j=0}^{\ell} \alpha_{j} \tilde{\chi}_{\ell-j} \quad(\ell \geq 1), \\
\tilde{\chi}_{\ell} & :=\sum_{\nu=1}^{\ell} \sum_{j \in \mathbb{N}^{\nu}} P_{j_{1}} \cdots P_{j_{v}} \chi_{0} \quad(\ell \geq 1)
\end{aligned}
$$


and

$$
\alpha_{0}=1, \quad \alpha_{\ell}:=-\frac{1}{2} \sum_{\substack{\boldsymbol{j} \in \mathbb{N}_{0}^{4} \\ j_{1}, j_{2}<\ell \\ \boldsymbol{j} \mid=\ell}} \alpha_{j_{1}} \alpha_{j_{2}}\left\langle\widetilde{\chi}_{j_{3}}, \tilde{\chi}_{j_{4}}\right\rangle \quad(\ell \geq 1)
$$

Before proving Theorem 4, let us first formally derive definition (B.3). Inserting the estimate (B.1) and the ansatz

$$
\chi=\sum_{\ell \geq 0} \varepsilon^{\ell} \chi_{\ell}
$$

into the equation $P \chi=\chi$ yields formally

$$
\sum_{\ell=0}^{\infty} \sum_{k=0}^{\ell} \varepsilon^{\ell} P_{k} \chi_{\ell-k}=\sum_{\ell=0}^{\infty} \varepsilon^{\ell} \chi_{\ell}
$$

hence

$$
\chi_{\ell}-P_{0} \chi_{\ell}=\sum_{k=1}^{\ell} P_{k} \chi_{\ell-k}
$$

and consequently

$$
\chi_{\ell}=\sum_{k=1}^{\ell} P_{k} \chi_{\ell-k}+\alpha_{\ell} \chi_{0}
$$

for any $\ell \geq 0$ and $\alpha_{\ell} \in \mathbb{C}, \alpha_{0}=1$. By induction over $\ell \in \mathbb{N}_{0}$, one easily verifies that $\chi_{\ell}$ can equivalently be written as definition (B.3a), with $\widetilde{\chi}_{\ell}$ given by definition (B.3b), without any further restriction on the parameters $\alpha_{\ell}$. It remains to derive definition (B.3c) for the (so far free) parameters $\alpha_{\ell}$. To this end, we observe that formally

$$
P=|\chi\rangle\left\langle\chi\left|=\sum_{\ell=0}^{\infty} \varepsilon^{\ell} \sum_{k=0}^{\ell}\right| \chi_{k}\right\rangle\left\langle\chi_{\ell-k}\right|,
$$

which motivates the definition

$$
P_{\ell}^{\mathrm{wf}}:=\sum_{k=0}^{\ell}\left|\chi_{k}\right\rangle\left\langle\chi_{\ell-k}\right|
$$

By definition (B.3a), this can equivalently be expressed as

$$
P_{\ell}^{\mathrm{wf}}=\sum_{k=0}^{\ell} \sum_{i=0}^{k} \sum_{m=0}^{\ell-k} \alpha_{i} \bar{\alpha}_{m}\left|\widetilde{\chi}_{k-i}\right\rangle\left\langle\widetilde{\chi}_{\ell-k-m}\left|=\sum_{\substack{\boldsymbol{j} \in \mathbb{N}_{0}^{4} \\|\boldsymbol{j}|=\ell}} \alpha_{j_{1}} \bar{\alpha}_{j_{2}}\right| \widetilde{\chi}_{j_{3}}\right\rangle\left\langle\widetilde{\chi}_{j_{4}}\right|
$$

Formally, it is clear that $P_{\ell}^{\mathrm{wf}}$ are the coefficients in the expansion of $P$, and our goal will be to rigorously establish the equality $P_{\ell}^{\mathrm{wf}}=P_{\ell}$. By the bound (B.1) and since $\operatorname{Tr}_{\mathfrak{S}} P_{0}=1$, it follows that

$$
1=\operatorname{Tr}_{\mathfrak{H}} P=1+\sum_{\ell=1}^{a} \varepsilon^{\ell} \operatorname{Tr}_{\mathfrak{H}} P_{\ell}+\mathcal{O}\left(\varepsilon^{a+1}\right),
$$


hence $\operatorname{Tr}_{\mathfrak{H}} P_{\ell}=0$ for any $\ell \geq 1$. Therefore, we choose the free parameters $\alpha_{\ell}$ such that $\operatorname{Tr}_{\mathfrak{H}} P_{\ell}^{\mathrm{wf}}=0$ for any $\ell \geq 0$, which implies that

$$
\alpha_{\ell}+\bar{\alpha}_{\ell}=-\sum_{\substack{\boldsymbol{j} \in \mathbb{N}_{0}^{4} \\ j_{1}, j_{2}<\ell \\|\boldsymbol{j}|=\ell}} \alpha_{j_{1}} \bar{\alpha}_{j_{2}}\left\langle\widetilde{\chi}_{j_{3}}, \widetilde{\chi}_{j_{4}}\right\rangle=0,
$$

and choosing $\alpha_{\ell}$ real results in definition (B.3c). Next, we prove an auxiliary lemma:

Lemma B.1. Under the assumptions of Theorem 4, it holds for any $\ell \in \mathbb{N}_{0}$ that

$$
P_{\ell}=\sum_{j=0}^{\ell} P_{j} P_{\ell-j}
$$

Proof. By assumption, it holds for any $a \in \mathbb{N}_{0}$ that

$$
P=\sum_{\ell=0}^{a} \varepsilon^{\ell} P_{\ell}+\varepsilon^{a+1} R_{a}
$$

for some $R_{a} \in \mathcal{L}(\mathfrak{H})$ with $\left\|R_{a}\right\|_{\mathrm{op}} \leq C(a)$. Since $P^{2}=P$, this implies that

$$
\sum_{\ell=0}^{a} \varepsilon^{\ell} P_{\ell}+\varepsilon^{a+1} R_{a}=\sum_{\ell=0}^{a} \varepsilon^{\ell}\left(\sum_{m=0}^{\ell} P_{m} P_{\ell-m}\right)+\varepsilon^{a+1} \widetilde{R}_{a}
$$

with

$$
\widetilde{R}_{a}=\sum_{\ell=0}^{a} \sum_{m=0}^{\ell-1} \varepsilon^{m} P_{\ell} P_{m+a+1-\ell}+\sum_{k=0}^{a} \varepsilon^{k}\left(R_{a} P_{k}+P_{k} R_{a}\right)+\varepsilon^{a+1} R_{a} R_{a}
$$

Consequently, it holds for any $a \in \mathbb{N}_{0}$ that

$$
\left\|\sum_{\ell=0}^{a} \varepsilon^{\ell}\left(P_{\ell}-\sum_{m=0}^{\ell} P_{m} P_{\ell-m}\right)\right\|_{\mathrm{op}} \leq \varepsilon^{a+1}\left\|R_{a}-\widetilde{R}_{a}\right\|_{\mathrm{op}} \leq C(a) \varepsilon^{a+1},
$$

and equation (B.13) follows by induction over $a \in \mathbb{N}$.

\section{Proof of Theorem 4}

We prove Theorem 4 in two steps: first, we show that the operators $P_{\ell}^{\mathrm{wf}}$ from definition (B.9), which are constructed from the ansatz (B.3) for the functions $\chi_{\ell}$, equal the coefficients $P_{\ell}$ in the expansion (B.1) of $P$; second, we estimate the difference between the truncated power series with coefficients $\chi_{\ell}$ and the function $\chi$.

Claim 1. Under the assumptions of Theorem 4, it holds for any $\ell \in \mathbb{N}_{0}$ that

$$
P_{\ell}^{w f}=P_{\ell}
$$

Proof. We prove equation (B.18) by induction over $\ell \in \mathbb{N}_{0}$. By Lemma B.1 and since $\operatorname{Tr}_{\mathfrak{H}} P_{1}=0$, we conclude that $\operatorname{Tr}_{\mathfrak{H}} P_{0} P_{1}=0$ and consequently $\alpha_{1}=0$. Hence, $\chi_{1}=\widetilde{\chi}_{1}=P_{1} \chi_{0}$, and definition (B.9) and 
Lemma B.1 imply that $P_{1}^{\mathrm{wf}}=P_{1}$. Now assume equation (B.18) for some $\ell \in \mathbb{N}$. Then, by equation (B.7),

$$
\begin{aligned}
P_{\ell+1}^{\mathrm{wf}}= & \sum_{k=0}^{\ell}\left|\chi_{k}\right\rangle\left\langle\chi_{\ell+1-k}|+| \chi_{\ell+1}\right\rangle\left\langle\chi_{0}\right| \\
= & \sum_{j=1}^{\ell+1} \sum_{k=0}^{\ell+1-j}\left|\chi_{k}\right\rangle\left\langle\chi_{\ell+1-k-j}\left|P_{j}+\sum_{k=1}^{\ell}\left(\alpha_{\ell+1-k}+P_{\ell+1-k}\right)\right| \chi_{k}\right\rangle\left\langle\chi_{0}\right| \\
& +2 \alpha_{\ell+1} P_{0}+P_{\ell+1} P_{0} \\
= & \sum_{j=1}^{\ell+1} P_{\ell+1-j}^{\mathrm{wf}} P_{j}+\sum_{k=1}^{\ell}\left(P_{\ell+1-k}+\alpha_{\ell+1-k}\right)\left|\chi_{k}\right\rangle\left\langle\chi_{0}\right| \\
& +2 \alpha_{\ell+1} P_{0}+P_{\ell+1} P_{0} .
\end{aligned}
$$

By the induction hypothesis and Lemma B.1,

$$
\sum_{j=1}^{\ell+1} P_{\ell+1-j}^{\mathrm{wf}} P_{j}+P_{\ell+1} P_{0}=\sum_{j=0}^{\ell+1} P_{\ell+1-j} P_{j}=P_{\ell+1},
$$

hence

$$
P_{\ell+1}^{\mathrm{wf}}=P_{\ell+1}+\sum_{k=1}^{\ell}\left(P_{\ell+1-k}+\alpha_{\ell+1-k}\right)\left|\chi_{k}\right\rangle\left\langle\chi_{0}\right|+2 \alpha_{\ell+1} P_{0}
$$

By construction, $\operatorname{Tr}_{\mathfrak{H}} P_{\ell}^{\mathrm{wf}}=\operatorname{Tr}_{\mathfrak{H}} P_{\ell}=0$ for any $\ell \geq 1$. Consequently, taking the trace of equation (B.21) yields

$$
\alpha_{\ell+1}=-\frac{1}{2} \sum_{k=1}^{\ell}\left\langle\chi_{0},\left(P_{\ell+1-k}+\alpha_{\ell+1-k}\right) \chi_{k}\right\rangle
$$

which implies that

$$
P_{\ell+1}^{\mathrm{wf}}=P_{\ell+1}+\left(1-P_{0}\right) \sum_{k=1}^{\ell}\left(P_{\ell+1-k}+\alpha_{\ell+1-k}\right)\left|\chi_{k}\right\rangle\left\langle\chi_{0}\right| .
$$

Finally,

$$
P_{0} P_{\ell+1}^{\mathrm{wf}}=P_{0} P_{\ell+1}, \quad P_{\ell+1}^{\mathrm{wf}}\left(1-P_{0}\right)=P_{\ell+1}\left(1-P_{0}\right)
$$

and, since both $P_{\ell+1}$ and $P_{\ell+1}^{\mathrm{wf}}$ are self-adjoint, the first equality implies that $P_{\ell+1}^{\mathrm{wf}} P_{0}=P_{\ell+1} P_{0}$. Adding this to the second equality in equation (B.24) concludes the proof of Claim 1.

Claim 2. Under the assumptions of Theorem 4, it holds for any $a \in \mathbb{N}_{0}$ that

$$
\left\|\chi-\sum_{\ell=0}^{a} \varepsilon^{\ell} \chi_{\ell}\right\|_{\mathfrak{H}} \leq \widetilde{C}(a) \varepsilon^{a+1} .
$$

Proof. By the bound (B.1), all operators $P_{\ell}$ are bounded uniformly in $\varepsilon$. Recall that for any normalised $f, g \in \mathfrak{H}$, it holds that $\left.\|f-g\|_{\mathfrak{H}} \leq 1 / \sqrt{2} \operatorname{Tr}_{\mathfrak{H}}|| f\right\rangle\langle f\rangle-|g\rangle\langle g||$ for a suitably chosen relative phase. By construction, the phase of all $\chi_{\ell}$ is determined by the phase of $\chi_{0}$. Hence, setting $n_{\varepsilon, a}:=\left\|\sum_{\ell=0}^{a} \varepsilon^{\ell} \chi_{\ell}\right\|^{-1}$, 
Claim 1 implies for a suitable choice of the phase of $\chi_{0}$ that

$$
\begin{aligned}
\left\|\chi-\sum_{\ell=0}^{a} \varepsilon^{\ell} \chi_{\ell}\right\|_{\mathfrak{H}} \leq & \left.\frac{1}{\sqrt{2}} \operatorname{Tr}_{\mathfrak{H}}\left|P-n_{\varepsilon, a}^{2} \sum_{\ell=0}^{a} \sum_{k=0}^{a} \varepsilon^{\ell+k}\right| \chi_{\ell}\right\rangle\left\langle\chi_{k}||+\left|\frac{1-n_{\varepsilon, a}}{n_{\varepsilon, a}}\right|\right. \\
\leq & \frac{1}{\sqrt{2}} \operatorname{Tr}_{\mathfrak{H}}\left|P-\sum_{\ell=0}^{a} \varepsilon^{\ell} P_{\ell}^{\mathrm{wf}}\right|+\frac{\varepsilon^{a+1}}{\sqrt{2}} \sum_{\ell=0}^{a} \sum_{j=1}^{\ell}\left\|\chi_{\ell}\right\|_{\mathfrak{H}}\left\|\chi_{a+j-\ell}\right\|_{\mathfrak{H}} \\
& +\left|\frac{1-n_{\varepsilon, a}^{2}}{\sqrt{2} n_{\varepsilon, a}^{2}}\right|+\left|\frac{1-n_{\varepsilon, a}}{n_{\varepsilon, a}}\right| \\
\leq & \widetilde{C}(a) \varepsilon^{a+1}
\end{aligned}
$$

by the bound (B.1) and definition (B.3). In addition, we use the fact that

$$
n_{\varepsilon, a}^{-2}=\operatorname{Tr}_{\mathfrak{H}}\left|\sum_{\ell=0}^{a} \varepsilon^{\ell} \chi_{\ell}\right\rangle\left\langle\sum_{k=0}^{a} \varepsilon^{k} \chi_{k}\right|=\operatorname{Tr}_{\mathfrak{H}}\left(\sum_{\ell=0}^{a} \varepsilon^{\ell} P_{\ell}\right)+\varepsilon^{a+1} R_{\varepsilon, a}=1+\varepsilon^{a+1} R_{\varepsilon, a},
$$

with

$$
R_{\varepsilon, a}=\sum_{\ell=0}^{a} \sum_{j=1}^{\ell} \varepsilon^{j-1} \operatorname{Tr}_{\mathfrak{H}}\left|\chi_{\ell}\right\rangle\left\langle\chi_{a+j-\ell}|, \quad| R_{\varepsilon, a}\right| \leq C(a)
$$

for some constant $C(a)$, which implies that $\left|\frac{1-n_{\varepsilon, a}^{2}}{n_{\varepsilon, a}^{2}}\right| \leq C(a) \varepsilon^{a+1}$, as well as $\left|\frac{1-n_{\varepsilon, a}}{n_{\varepsilon, a}}\right| \leq C(a) \varepsilon^{a+1}$.

Acknowledgments. The first author gratefully acknowledges funding from the European Union's Horizon 2020 research and innovation programme under Marie Skłodowska-Curie Grant Agreement No. 754411. The third author was supported by the European Research Council (ERC) under the European Union's Horizon 2020 research and innovation programme (Grant Agreement No. 694227).

\section{Conflict of Interest: None.}

\section{References}

[1] A. Adhikari, C. Brennecke and B. Schlein, 'Bose-Einstein condensation beyond the Gross-Pitaevskii regime', Ann. Henri Poincaré (NN ) (2020).

[2] S. Beliaev, 'Application of the methods of quantum field theory to a system of bosons', Sov. Phys. J. Exper. Theoret. Phys., 34(2) (1958), 289-299.

[3] S. Beliaev, 'Energy spectrum of a non-ideal Bose gas', Sov. Phys. J. Exper. Theoret. Phys., 34(2) (1958), $299-307$.

[4] C. Boccato, C. Brennecke, S. Cenatiempo and B. Schlein, 'Complete Bose-Einstein condensation in the Gross-Pitaevskii regime', Comm. Math. Phys. 359(3) (2018), 975-1026.

[5] C. Boccato, C. Brennecke, S. Cenatiempo and B. Schlein, 'Bogoliubov theory in the Gross-Pitaevskii limit', Acta Math. 222(2) (2019), 219-335.

[6] C. Boccato, C. Brennecke, S. Cenatiempo and B. Schlein, 'The excitation spectrum of Bose gases interacting through singular potentials', J. Eur. Math. Soc. (JEMS) 22(7) (2020), 2331-2403.

[7] C. Boccato, C. Brennecke, S. Cenatiempo and B. Schlein, 'Optimal rate for Bose-Einstein condensation in the GrossPitaevskii regime', Comm. Math. Phys. 376 (2020), 1311-1395.

[8] C. Boccato, S. Cenatiempo and B. Schlein, 'Quantum many-body fluctuations around nonlinear Schrödinger dynamics', Ann. Henri Poincaré 18(1) (2017), 113-191.

[9] N. N. Bogoliubov, 'On the theory of superfluidity', Izv. Akad. Nauk Ser. Fiz. 11 (1947), 23-32.

[10] L. Boßmann, N. Pavlović, P. Pickl and A. Soffer, 'Higher order corrections to the mean-field description of the dynamics of interacting bosons', J. Stat. Phys. 178(6) (2020), 1362-1396.

[11] L. Boßmann, S. Petrat, P. Pickl and A. Soffer, 'Beyond Bogoliubov dynamics', Preprint, 2019, arXiv:1912.11004.

[12] E. Braaten, H.-W. Hammer and S. Hermans, 'Nonuniversal effects in the homogeneous Bose gas', Phys. Rev. A 63(6) (2001), 063609. 
[13] E. Braaten and A. Nieto, 'Quantum corrections to the energy density of a homogeneous Bose gas', Euro. Phys. J. B 11(1) (1999), 143-159.

[14] C. Brennecke, P. T. Nam, M. Napiórkowski and B. Schlein, 'Fluctuations of N-particle quantum dynamics around the nonlinear Schrödinger equation', Ann. Inst. H. Poincaré Anal. Non Linéaire 36(5) (2019), 1201-1235.

[15] B. Brietzke, S. Fournais and J. P. Solovej, 'A simple 2nd order lower bound to the energy of dilute Bose gases', Comm. Math. Phys. 376 (2020), 323-351.

[16] B. Brietzke and J. P. Solovej, 'The second-order correction to the ground state energy of the dilute Bose gas', Ann. Henri Poincaré 21 (2020), 571-626.

[17] K. Brueckner and K. Sawada, 'Bose-Einstein gas with repulsive interactions: General theory', Phys. Rev. 106(6) (1957), 1117-1127.

[18] K. Brueckner and K. Sawada, 'Bose-Einstein gas with repulsive interactions: Hard spheres at high density', Phys. Rev. 106(6) (1957), 1128-1135.

[19] S. Cenatiempo and A. Giuliani, 'Renormalization theory of a two dimensional Bose gas: Quantum critical point and quasicondensed state', J. Stat. Phys. 157 (2014), 755-829.

[20] J. Chong, 'Dynamics of large boson systems with attractive interaction and a derivation of the cubic focusing NLS in $\mathbb{R}^{3}$, Preprint, 2016, arXiv:1608.01615.

[21] J. Dereziński and M. Napiórkowski, 'Excitation spectrum of interacting bosons in the mean-field infinite-volume limit', Ann. Henri Poincaré 15(12) (2014), 2409-2439.

[22] L. Erdős, B. Schlein and H.-T. Yau, 'Ground-state energy of a low-density Bose gas: A second-order upper bound', Phys. Rev. A 78 (2008).

[23] S. Fournais and J. P. Solovej, 'The energy of dilute Bose gases', Ann. Math. 192(3) (2020), 893-976.

[24] J. Ginibre and G. Velo, 'The classical field limit of non-relativistic bosons. II. Asymptotic expansions for general potentials', Ann. Inst. H. Poincaré Phys. Théor. 33(4) (1980), 363-394.

[25] J. Ginibre and G. Velo, 'The classical field limit of nonrelativistic bosons. I. Borel summability for bounded potentials', Ann. Phys. 128(2) (1980), 243-285.

[26] A. Giuliani and R. Seiringer, 'The ground state energy of the weakly interacting Bose gas at high density', J. Stat. Phys. 135 (2009), 915-934.

[27] P. Grech and R. Seiringer, 'The excitation spectrum for weakly interacting bosons in a trap', Comm. Math. Phys. 322(2) (2013), 559-591.

[28] M. Grillakis and M. Machedon, 'Pair excitations and the mean field approximation of interacting bosons, I', Comm. Math. Phys. 324(2) (2013), 601-636.

[29] M. Grillakis and M. Machedon, 'Pair excitations and the mean field approximation of interacting bosons, II', Comm. Partial Differential Equations 42(1) (2017), 24-67.

[30] M. Grillakis, M. Machedon and D. Margetis, 'Second-order corrections to mean field evolution of weakly interacting bosons, I', Comm. Math. Phys. 294(1) (2010), 273-301.

[31] M. Grillakis, M. Machedon and D. Margetis, 'Second-order corrections to mean field evolution of weakly interacting bosons, II', Adv. Math. 228(3) (2011), 1788-1815.

[32] N. Hugenholtz and D. Pines, 'Ground-state energy and excitation spectrum of a system of interacting bosons', Phys. Rev. 116(3) (1959), 489-506.

[33] E. Kuz, 'Exact evolution versus mean field with second-order correction for bosons interacting via short-range two-body potential', Differential Integral Equations 30(7/8) (2017), 587-630.

[34] M. Lewin, P. T. Nam and N. Rougerie, 'Derivation of Hartree's theory for generic mean-field Bose systems', Adv. Math. 254 (2014), 570-621.

[35] M. Lewin, P. T. Nam and B. Schlein, 'Fluctuations around Hartree states in the mean field regime', Amer. J. Math. 137(6) (2015), 1613-1650.

[36] M. Lewin, P. T. Nam, S. Serfaty and J. P. Solovej, 'Bogoliubov spectrum of interacting Bose gases', Comm. Pure Appl. Math. 68(3) (2015), 413-471.

[37] E. H. Lieb and R. Seiringer, 'Proof of Bose-Einstein condensation for dilute trapped gases', Phys. Rev. Lett. 88(17) (2002), 170409.

[38] E. H. Lieb and R. Seiringer, 'Derivation of the Gross-Pitaevskii equation for rotating Bose gases', Comm. Math. Phys. 264(2) (2006), 505-537.

[39] E. H. Lieb, R. Seiringer, J. P. Solovej and J. Yngvason, The Mathematics of the Bose Gas and Its Condensation (Birkhäuser, Basel, 2005).

[40] E. H. Lieb, R. Seiringer and J. Yngvason, 'Bosons in a trap: A rigorous derivation of the Gross-Pitaevskii energy functional', Phys. Rev. A 61(4) (2000), 043602.

[41] E. H. Lieb and J. P. Solovej, 'Ground state energy of the one-component charged Bose gas', Comm. Math. Phys. 217 (2001), 127-163.

[42] E. H. Lieb and J. P. Solovej, 'Ground state energy of the two-component charged Bose gas', Comm. Math. Phys. 252 (2004), 485-534.

[43] E. H. Lieb and J. Yngvason, 'Ground state energy of the low density Bose gas', Phys. Rev. Lett. 80(12) (1998), $2504-2507$. 
[44] D. Mitrouskas, Derivation of Mean Field Equations and Their Next-Order Corrections: Bosons and Fermions, PhD thesis, LMU Munich, 2017.

[45] D. Mitrouskas, S. Petrat and P. Pickl, 'Bogoliubov corrections and trace norm convergence for the Hartree dynamics', Rev. Math. Phys. 31(8) (2019), 1-36.

[46] P. T. Nam, 'Bogoliubov theory and bosonic atoms', Preprint, 2011, arXiv:1109.2875.

[47] P. T. Nam, Contributions to the Rigorous Study of the Structure of Atoms, PhD thesis, University of Copenhagen, 2011.

[48] P. T. Nam, 'Binding energy of homogeneous Bose gases', Lett. Math. Phys. 108(1) (2018), 141-159.

[49] P. T. Nam and M. Napiórkowski, 'Bogoliubov correction to the mean-field dynamics of interacting bosons', Adv. Theor. Math. Phys. 21(3) (2017), 683-738.

[50] P. T. Nam and M. Napiórkowski, 'A note on the validity of Bogoliubov correction to mean-field dynamics', J. Math. Pures Appl. (9) 108(5) (2017), 662-688.

[51] P. T. Nam and M. Napiórkowski, 'Two-term expansion of the ground state one-body density matrix of a mean-field Bose gas', Preprint, 2020, arXiv:2010.03595.

[52] P. T. Nam, M. Napiórkowski, J. Ricaud and A. Triay, 'Optimal rate of condensation for trapped bosons in the Gross-Pitaevskii regime', Preprint, 2020, arXiv:2001.04364.

[53] P. T. Nam, M. Napiórkowski and J. P. Solovej, 'Diagonalization of bosonic quadratic Hamiltonians by Bogoliubov transformations', J. Funct. Anal. 270(11) (2016), 4340-4368.

[54] P. T. Nam, N. Rougerie and R. Seiringer, 'Ground states of large bosonic systems: The Gross-Pitaevskii limit revisited', Anal. PDE 9(2) (2016), 459-485.

[55] P. T. Nam and R. Seiringer, 'Collective excitations of Bose gases in the mean-field regime', Arch. Ration. Mech. Anal. 215 (2015), 381-417.

[56] M. Napiórkowski, 'Recent advances in the theory of Bogoliubov Hamiltonians' in Workshop on Macroscopic Limits of Quantum Systems (Springer, Heidelberg, 2017), 101-121.

[57] T. Paul and M. Pulvirenti, 'Asymptotic expansion of the mean-field approximation', Discrete Contin. Dyn. Syst. 39(4) (2019), 1891-1921.

[58] S. Petrat, P. Pickl and A. Soffer, 'Derivation of the Bogoliubov time evolution for a large volume mean-field limit', Ann. Henri Poincaré, 21(2) (2020), 461-498.

[59] A. Pizzo, 'Bose particles in a box I. A convergent expansion of the ground state of a three-modes Bogoliubov Hamiltonian', Preprint, 2015, arXiv:1511.07022.

[60] A. Pizzo, 'Bose particles in a box II. A convergent expansion of the ground state of the Bogoliubov Hamiltonian in the mean field limiting regime', Preprint, 2015, arXiv:1511.07025.

[61] A. Pizzo, 'Bose particles in a box III. A convergent expansion of the ground state of the Hamiltonian in the mean field limiting regime', Preprint, 2015, arXiv:1511.07026.

[62] M. Reed and B. Simon, Methods of Modern Mathematical Physics, Vol. IV: Analysis of Operators (Academic Press, London, 1978).

[63] J. Sakurai and J. Napolitano, Modern Quantum Mechanics, second edn (Person New International, San Fransico, 2014).

[64] K. Sawada, 'Ground-state energy of Bose-Einstein gas with repulsive interaction', Phys. Rev. 116(6) (1959), 1344-1358.

[65] R. Seiringer, 'The excitation spectrum for weakly interacting bosons', Comm. Math. Phys. 306(2) (2011), 565-578.

[66] J. P. Solovej, 'Upper bounds to the ground state energies of the one- and two-component charged Bose gases', Comm. Math. Phys. 266 (2006), 797-818.

[67] J. P. Solovej, 'Many body quantum mechanics', unpublished notes (2007). URL: http://www.mathematik.uni-muenchen.de/ $\sim$ sorensen/Lehre/SoSe2013/MQM2/skript.pdf.

[68] C. Weiss and A. Eckardt, 'Ground state energy of a homogeneous Bose-Einstein condensate beyond Bogoliubov', Europhys. Lett. 68(1) (2004), 8-14.

[69] T. T. Wu, 'Ground state of a Bose system of hard spheres', Phys. Rev. 115(6) (1959), 1390-1404.

[70] H.-T. Yau and J. Yin, 'The second order upper bound for the ground energy of a Bose gas', J. Stat. Phys. 136 (2009), $453-503$. 